\title{
Théorie d'Iwasawa des représentations cristallines II
}

\author{
Denis Benois et Laurent Berger
}

Résumé. Soit $K$ une extension finie non-ramifiée de $\mathbb{Q}_{p}$ et $V$ une représentation cristalline de $\operatorname{Gal}\left(\overline{\mathbb{Q}}_{p} / K\right)$. Dans cet article, on montre la conjecture $C_{\mathrm{EP}}(L, V)$ pour $L \subset \mathbb{Q}_{p}^{\mathrm{ab}}$ et sa version équivariante $C_{\mathrm{EP}}(L / K, V)$ pour $L \subset \bigcup_{n=1}^{\infty} K\left(\zeta_{p^{n}}\right)$. Les principaux ingrédients sont la conjecture $\delta_{\mathbb{Z}_{p}}(V)$ sur l'intégralité de l'exponentielle de Perrin-Riou, que nous démontrons en utilisant la théorie des $(\varphi, \Gamma)$-modules, et des techniques de descente en théorie d'Iwasawa pour montrer que $\delta_{\mathbb{Z}_{p}}(V)$ implique $C_{\mathrm{EP}}(L / K, V)$.

\begin{abstract}
Let $K$ be a finite unramified extension of $\mathbb{Q}_{p}$ and let $V$ be a crystalline representation of $\operatorname{Gal}\left(\overline{\mathbb{Q}}_{p} / K\right)$. In this article, we give a proof of the $C_{\mathrm{EP}}(L, V)$ conjecture for $L \subset \mathbb{Q}_{p}^{\text {ab }}$ as well as a proof of its equivariant version $C_{\mathrm{EP}}(L / K, V)$ for $L \subset \bigcup_{n=1}^{\infty} K\left(\zeta_{p^{n}}\right)$. The main ingredients are the $\delta_{\mathbb{Z}_{p}}(V)$ conjecture about the integrality of Perrin-Riou's exponential, which we prove using the theory of $(\varphi, \Gamma)$-modules, and Iwasawa-theoretic descent techniques used to show that $\delta_{\mathbb{Z}_{p}}(V)$ implies $C_{\mathrm{EP}}(L / K, V)$.
\end{abstract}

Mathematics Subject Classification (2000). 11F80, 11R23, 11S15, 11S20, 11S25, 14F30.

Keywords. Iwasawa theory, $p$-adic representations, Tamagawa numbers.

\section{Table des matières}

Introduction . . . . . . . . . . . . . . . . . . . . . . 604

1 Représentations potentiellement semi-stables . . . . . . . . . . . . 607

1.1 Théorie de Hodge $p$-adique . . . . . . . . . . . . . . . . 608

1.2 Modules de Wach et $(\varphi, \Gamma)$-modules . . . . . . . . . . . . . . . . 609

1.3 Cohomologie galoisienne . . . . . . . . . . . . . . 610

1.4 L'exponentielle de Bloch-Kato . . . . . . . . . . . . . . . . 611

2 Déterminants et constantes locales . . . . . . . . . . . . . . . . 614

2.1 Déterminants généralisés . . . . . . . . . . . . . . . 614

2.2 Déterminants de la cohomologie galoisienne . . . . . . . . . 619

2.3 Constantes locales des représentations de Weil-Deligne . . . . . . . 621

2.4 Constantes locales des représentations potentiellement semi-stables . 624

2.5 La conjecture $C_{\mathrm{EP}}(L / K, V)$. . . . . . . . . . . . . . . . . . 629 
3 L'exponentielle de Perrin-Riou . . . . . . . . . . . . . . . . . . . . 632

3.1 Rappels et compléments . . . . . . . . . . . . . . . . . . . 632

3.2 L'application exponentielle et les $(\varphi, \Gamma)$-modules . . . . . . . . 635

4 La conjecture $C_{\mathrm{Iw}}\left(K_{\infty} / K, V\right) \ldots \ldots \ldots \ldots 64 \ldots \ldots \ldots$

4.1 Enoncé de la conjecture . . . . . . . . . . . . . . . . . . . . 643

4.2 Équivalence de $C_{\mathrm{Iw}}$ et de $C_{\mathrm{EP}}$ : étude de $\Xi_{V, n}^{\varepsilon} \ldots \ldots \ldots 64$

4.3 Équivalence de $C_{\mathrm{Iw}}$ et de $C_{\mathrm{EP}}$ : étude de $\operatorname{Exp}_{V, h, n}^{\varepsilon} \ldots \ldots \ldots 68$

4.4 Résultats principaux . . . . . . . . . . . . . . . . 670

Références . . . . . . . . . . . . . . . . . . 675

\section{Introduction}

Soient $p$ un nombre premier impair, $K$ une extension finie de $\mathbb{Q}_{p}$ et $V$ une représentation potentiellement semi-stable de $G_{K}=\operatorname{Gal}\left(\overline{\mathbb{Q}}_{p} / K\right)$. Fontaine et Perrin-Riou ont formulé une conjecture qu'ils ont appelée $C_{\mathrm{EP}}(K, V)$, conjecture qui entraîne la compatibilité de la conjecture de Bloch et Kato sur les valeurs spéciales des fonctions $L$ avec l'équation fonctionnelle. L'objet de ce texte est de montrer la conjecture $C_{\mathrm{EP}}(L, V)$ pour toute extension finie $L$ de $K$ telle que $L \subset \mathbb{Q}_{p}^{\mathrm{ab}}$, quand $K$ est nonramifié sur $\mathbb{Q}_{p}$ et $V$ est une représentation cristalline de $G_{K}$ ainsi que, sous les mêmes hypothèses, la version équivariante $C_{\mathrm{EP}}(L / K, V)$ de cette conjecture pour toute extension finie $L$ de $K$ contenue dans $K_{\infty}=\bigcup_{n=1}^{\infty} K\left(\zeta_{p^{n}}\right)$. Comme ingrédient de la démonstration, on montre aussi la conjecture $\delta_{\mathbb{Z}_{p}}(V)$ de Perrin-Riou, que nous appelons $C_{\mathrm{Iw}}\left(K_{\infty} / K, V\right)$ en raison de son lien avec la théorie d'Iwasawa de $V$.

Rappelons tout d'abord la conjecture $C_{\mathrm{EP}}(L / K, V)$. Pour cela, on se donne une extension abélienne finie $L / K$ de groupe de Galois $G=\operatorname{Gal}(L / K)$, une représentation potentiellement semi-stable $V$ de $G_{K}$ et un réseau $T$ de $V$ stable sous l'action de $G_{K}$. On définit la droite d'Euler-Poincaré de $V$ en posant :

$$
\Delta_{\mathrm{EP}}(L / K, V)=\operatorname{det}_{\mathbb{Q}_{p}[G]} \mathbb{R} \Gamma(L, V) \otimes \operatorname{det}_{\mathbb{Q}_{p}[G]}\left(\operatorname{Ind}_{L / \mathbb{Q}_{p}} V\right) .
$$

On sait que $\mathbb{R} \Gamma(L, T)$ est un complexe parfait de $\mathbb{Z}_{p}[G]$-modules et que l'image de $\Delta_{\mathrm{EP}}(L / K, T)=\operatorname{det}_{\mathbb{Z}_{p}[G]} \mathbb{R} \Gamma(L, T) \otimes \operatorname{det}_{\mathbb{Z}_{p}[G]}\left(\operatorname{Ind}_{L / \mathbb{Q}_{p}} T\right)$ dans $\Delta_{\mathrm{EP}}(L / K, V)$ ne dépend pas du choix de $T$.

On note $\boldsymbol{D}_{\text {cris }}^{L}(V), \boldsymbol{D}_{\mathrm{pst}}(V)$ et $\boldsymbol{D}_{\mathrm{dR}}^{L}(V)$ les modules associés à la restriction de $V$ à $G_{L}$ par la théorie de Fontaine, et $t_{V}(L)=\boldsymbol{D}_{\mathrm{dR}}^{L}(V) / \mathrm{Fil}^{0} \boldsymbol{D}_{\mathrm{dR}}^{L}(V)$ l'espace tangent de $V$ sur $L$. La suite exacte :

$$
\begin{aligned}
0 \rightarrow H^{0}(L, V) & \rightarrow D_{\text {cris }}^{L}(V) \rightarrow D_{\text {cris }}^{L}(V) \oplus t_{V}(L) \rightarrow H^{1}(L, V) \rightarrow \\
& \rightarrow D_{\text {cris }}^{L}\left(V^{*}(1)\right)^{*} \oplus t_{V^{*}(1)}^{*}(L) \rightarrow D_{\text {cris }}^{L}\left(V^{*}(1)\right)^{*} \rightarrow H^{2}(L, V) \rightarrow 0,
\end{aligned}
$$


qui provient de la suite exacte fondamentale (cf. §1.4) et l'isomorphisme $t_{V^{*}(1)}^{*}(L) \simeq$ $\mathrm{Fil}^{0} \boldsymbol{D}_{\mathrm{dR}}^{L}(V)$ donnent un isomorphisme canonique :

$$
\operatorname{det}_{\mathbb{Q}_{p}[G]} \mathbb{R} \Gamma(L, V) \stackrel{\sim}{\rightarrow} \operatorname{det}_{\mathbb{Q}_{p}[G]}^{-1} D_{\mathrm{dR}}^{L}(V)
$$

La théorie des constantes locales permet d'autre part de définir un élément $\varepsilon(L / K, V) \in \mathbb{Q}_{p}\left(\zeta_{p^{\infty}}\right)[G]$ associé à l'action de $G_{K}$ sur $\boldsymbol{D}_{\mathrm{pst}}(V)$. L'isomorphisme de comparaison :

$$
\boldsymbol{B}_{\mathrm{dR}} \otimes_{\mathbb{Q}_{p}} \operatorname{Ind}_{L / \mathbb{Q}_{p}}(V) \simeq \boldsymbol{B}_{\mathrm{dR}} \otimes_{\mathbb{Q}_{p}} \boldsymbol{D}_{\mathrm{dR}}^{L}(V),
$$

normalisé par $\varepsilon(L / K, V)$ et par le facteur $\Gamma$ habituel $\Gamma^{*}(V)$, fournit un isomorphisme :

$$
\operatorname{det}_{\mathbb{Q}_{p}[G]}^{-1} D_{\mathrm{dR}}^{L}(V) \otimes \operatorname{det}_{\mathbb{Q}_{p}[G]} \operatorname{Ind}_{L / \mathbb{Q}_{p}}(V) \simeq \mathbb{Q}_{p}[G]_{V, L / K},
$$

où $\mathbb{Q}_{p}[G]_{V, L / K}$ est un certain $\mathbb{Q}_{p}[G]$-module libre de rang 1 qui contient un sous$\mathbb{Z}_{p}[G]$-module inversible canonique $\mathbb{Z}_{p}[G]_{V, L / K}$ (cf. définition 2.14). En composant ces isomorphismes, on obtient une trivialisation canonique de la droite d'EulerPoincaré :

$$
\delta_{V, L / K}: \Delta_{\mathrm{EP}}(L / K, V) \simeq \mathbb{Q}_{p}[G]_{V, L / K} .
$$

Dans son manuscrit non-publié [Kat93b], Kato a proposé la conjecture suivante (qu'il appelle «local $\varepsilon$-conjecture ») :

Conjecture $\boldsymbol{C}_{\mathbf{E P}}(\boldsymbol{L} / \mathrm{K}, \boldsymbol{V})$. Si $V$ est une représentation potentiellement semi-stable et si $L / K$ est une extension abélienne finie, alors l'application $\delta_{V, L / K}$ envoie $\Delta_{\mathrm{EP}}(L / K, T)$ sur $\mathbb{Z}_{p}[G]_{V, L / K}$.

C'est la conjecture 2.19 de cet article. Si $L=K$, alors on retrouve la conjecture $C_{\mathrm{EP}}(K, V)$ de Fontaine et Perrin-Riou que l'on peut d'ailleurs reformuler en termes de nombres de Tamagawa (cf. conjecture 2.20).

Rappelons à présent la conjecture $C_{\mathrm{Iw}}\left(K_{\infty} / K, V\right)$. On suppose pour cela que $K$ est non-ramifié, on fixe une suite compatible de racines primitives $p^{n}$-ièmes de l'unité $\varepsilon=\left(\zeta_{p^{n}}\right)_{n \geqslant 0}$ et pour $n \geqslant 1$, on pose $K_{n}=K\left(\zeta_{p^{n}}\right)$ ainsi que $K_{\infty}=\bigcup_{n \geqslant 1} K_{n}$. Soient $H_{K}=\operatorname{Gal}\left(\overline{\mathbb{Q}}_{p} / K_{\infty}\right), \Gamma=\operatorname{Gal}\left(K_{\infty} / K\right)$ et $\Gamma_{n}=\operatorname{Gal}\left(K_{\infty} / K_{n}\right)$ ce qui fait que $\Gamma=\Delta_{K} \times \Gamma_{1}$ où $\Delta_{K}$ est le sous-groupe de torsion de $\Gamma$. Soit $\mathcal{H}$ l'algèbre des séries formelles $f(X) \in \mathbb{Q}_{p}[[X]]$ qui convergent sur le disque unité ouvert et $\mathscr{H}\left(\Gamma_{1}\right)=\left\{f\left(\gamma_{1}-1\right) \mid \gamma_{1} \in \Gamma_{1}\right.$ et $\left.f \in \mathscr{H}\right\}$. On pose $\Lambda=\mathbb{Z}_{p}[[\Gamma]], \mathscr{H}(\Gamma)=$ $\mathbb{Q}_{p}\left[\Delta_{K}\right] \otimes_{\mathbb{Q}_{p}} \mathscr{H}\left(\Gamma_{1}\right)$ et $\mathcal{K}(\Gamma)$ est l'anneau total des fractions de $\mathscr{H}(\Gamma)$. On définit la cohomologie d'Iwasawa d'une représentation $V$ en posant :

$$
H_{\mathrm{Iw}}^{1}(K, T)={\underset{\operatorname{cor}}{K_{n} / K_{n-1}}}_{\lim _{n}} H^{1}\left(K_{n}, T\right)
$$


et $H_{\mathrm{IW}}^{1}(K, V)=\mathbb{Q}_{p} \otimes_{\mathbb{Z}_{p}} H_{\mathrm{Iw}}^{1}(K, T)$.

Supposons à présent que $V$ est cristalline. Dans [Per94], Perrin-Riou a construit une famille d'applications :

$$
\operatorname{Exp}_{V, h}^{\varepsilon}: \mathscr{D}(V)^{\Delta=0} \rightarrow \mathscr{H}(\Gamma) \otimes_{\Lambda} H_{\mathrm{Iw}}^{1}(K, V) / V^{H_{K}},
$$

qui interpolent les exponentielles de Bloch et Kato. Plus précisement, pour tout $h \geqslant 1$ vérifiant $\mathrm{Fil}^{-h} \boldsymbol{D}_{\mathrm{dR}}(V)=\boldsymbol{D}_{\mathrm{dR}}(V)$, on a un diagramme commutatif :

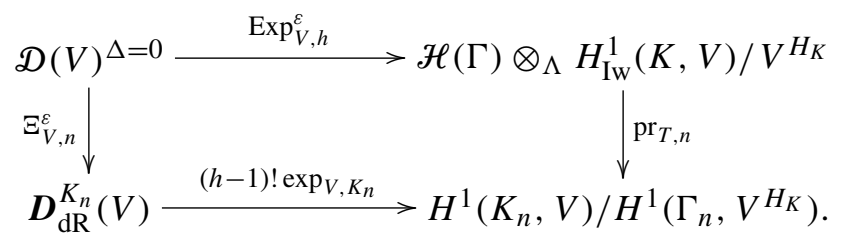

Ici, $\mathscr{D}(V)$ est isomorphe à $\Lambda \otimes_{\mathbb{Z}_{p}} \boldsymbol{D}_{\text {cris }}(V)$ et les applications $\Delta$ et $\Xi_{V, n}^{\varepsilon}$ sont explicites, mais leur définition est un peu technique pour cette introduction (cf. paragraphe 3.1). Cette construction joue un rôle important dans la théorie des fonctions $L p$-adiques (cf. [Per95] et [Col99b]). Posons maintenant :

$$
\Delta_{\mathrm{Iw}}\left(K_{\infty} / K, T\right)=\operatorname{det}_{\Lambda} \mathbb{R} \Gamma_{\mathrm{Iw}}(K, T) \otimes \operatorname{det}_{\Lambda}\left(\operatorname{Ind}_{K_{\infty} / \mathbb{Q}_{p}} T\right),
$$

et $\Delta_{\mathrm{Iw}}\left(K_{\infty} / K, V\right)=\mathbb{Q}_{p} \otimes_{\mathbb{Z}_{p}} \Delta_{\mathrm{Iw}}\left(K_{\infty} K, T\right)$. On pose $\ell_{j}=j-\log \gamma_{1} / \log \chi\left(\gamma_{1}\right)$ et on définit un facteur $\Gamma$ par la formule :

$$
\boldsymbol{\Gamma}_{h}(V)=\prod_{j>-h}\left(\ell_{-j}\right)^{\operatorname{dim}_{\mathbb{Q}_{p}} \operatorname{Fil}^{j} \boldsymbol{D}_{\text {cris }}(V)}
$$

Le déterminant de $\operatorname{Exp}_{V, h}^{\varepsilon}$ normalisé par $\Gamma_{h}(V)^{-1}$ ne dépend alors pas de $h$, et la loi de réciprocité de Perrin-Riou entraîne qu'il induit un isomorphisme canonique :

$$
\delta_{V, K_{\infty} / K}: \Delta_{\mathrm{Iw}}\left(K_{\infty} / K, V\right) \rightarrow \mathbb{Q}_{p} \otimes_{\mathbb{Z}_{p}} \Lambda_{V, K_{\infty} / K},
$$

où $\Lambda_{V, K_{\infty} / K}$ est un certain $\Lambda$-module libre de rang 1 (cf. le paragraphe 4.1). PerrinRiou a proposé la conjecture suivante (appelée $\delta_{\mathbb{Z}_{p}}(V)$ dans [Per94] et [Per95]) relativement au déterminant de $\operatorname{Exp}_{V, h}^{\varepsilon}$.

Conjecture $C_{\mathrm{Iw}}\left(K_{\infty} / K, V\right)$. Si $V$ est une représentation cristalline de $G_{K}$, alors l'application $\delta_{V, K_{\infty} / K}$ envoie $\Delta_{\mathrm{Iw}}\left(K_{\infty} / K, T\right)$ sur $\Lambda_{V, K_{\infty} / K}$.

Le résultat principal de cet article est le suivant :

Théorème A. Si $K$ est une extension non-ramifiée de $\mathbb{Q}_{p}$ et si $V$ est une représentation cristalline de $G_{K}$, alors : 
(1) la conjecture $C_{\mathrm{IW}}\left(K_{\infty} / K, V\right)$ est vraie;

(2) la conjecture $C_{\mathrm{EP}}(L / K, V)$ est vraie pour toute extension finie $L$ de $K$ contenue dans $K_{\infty}$.

En utilisant les propriétés fonctorielles de la conjecture $C_{\mathrm{EP}}(L / K, V)$, on en déduit le corollaire suivant :

Corollaire B. Si K est une extension non-ramifiée de $\mathbb{Q}_{p}$ et si $V$ est une représentation cristalline de $G_{K}$, alors :

(1) la conjecture $C_{\mathrm{EP}}(L, V)$ est vraie pour toute extension $L / K$ contenue dans $\mathbb{Q}_{p}^{\mathrm{ab}}$;

(2) la conjecture $C_{\mathrm{EP}}(K, V(\eta))$ est vraie pour tout caractère de Dirichlet $\eta$ de $\Gamma$.

Le théorème $\mathrm{A}$ et le corollaire $\mathrm{B}$ sont démontrés à la fin de cet article (cf. le théorème 4.22 et le corollaire 4.23). Disons quelques mots du plan de l'article. Les chapitres 1 et 2 sont consacrés à des rappels, qui aboutissent à l'énoncé de la conjecture $C_{\mathrm{EP}}(L / K, V)$. Les chapitres 3 et 4 sont le coeur technique de l'article. On commence par y rappeler la construction de l'exponentielle de Perrin-Riou, puis on y énonce la conjecture $C_{\mathrm{IW}}\left(K_{\infty} / K, V\right)$. Après cela on montre dans les paragraphes $\S \S 4.2$, 4.3, en utilisant des techniques de descente en théorie d'Iwasawa, que la conjecture $C_{\mathrm{Iw}}\left(K_{\infty} / K, V\right)$ est équivalente à la conjecture $C_{\mathrm{EP}}\left(K_{n} / K, V\right)$ pour tout $n \geqslant 1$. Enfin dans le $\$ 4.4$ on démontre la conjecture $C_{\mathrm{Iw}}\left(K_{\infty} / K, V\right)$.

Les mêmes arguments, avec un peu plus de calculs, permettent de démontrer la conjecture $C_{\mathrm{EP}}(L / K, V)$ pour toute extension $L / K$ contenue dans $\mathbb{Q}_{p}^{\mathrm{ab}}$. Cette petite généralisation est importante pour la version équivariante des conjectures de Bloch et Kato ; nous en laissons les détails au lecteur.

Pour terminer cette introduction, remarquons que dans le cas où $V$ est ordinaire, ces résultats étaient déjà connus (voir [Per94], [BN02], [BF04]).

Remerciements. Nous remercions Pierre Colmez pour avoir attiré notre attention sur ce problème et nous avoir encouragés au long de notre travail. Nous remercions aussi le rapporteur pour sa lecture minutieuse du texte qui nous a permis de beaucoup l'améliorer.

\section{Représentations potentiellement semi-stables}

Dans tout cet article, le corps $K$ est une extension finie de $\mathbb{Q}_{p}$ (dans les chapitres 3 et 4, on suppose qu'elle est non-ramifiée). L'anneau des entiers de $K$ est noté $\mathcal{O}_{K}$ et son corps résiduel $k_{K}$ est de cardinal $q_{K}$. On fixe une fois pour toutes une suite compatible de racines primitives $p^{n}$-ièmes de l'unité $\varepsilon=\left(\zeta_{p^{n}}\right)_{n \geqslant 0}$ et pour $n \geqslant 1$, on 
pose $K_{n}=K\left(\zeta_{p^{n}}\right)$ ainsi que $K_{\infty}=\bigcup_{n \geqslant 1} K_{n}$. La notation $K_{0}$ désigne le sous-corps maximal non-ramifié de $K$.

On pose :

$$
\begin{array}{rlrl}
G_{K} & =\operatorname{Gal}\left(\overline{\mathbb{Q}}_{p} / K\right), & H_{K} & =\operatorname{Gal}\left(\overline{\mathbb{Q}}_{p} / K_{\infty}\right), \\
\Gamma_{n} & =\operatorname{Gal}\left(K_{\infty} / K_{n}\right), \quad G_{n}=\operatorname{Gal}\left(K_{n} / K\right),
\end{array}
$$

et $\Lambda=\mathbb{Z}_{p}[[\Gamma]]$ est l'algèbre d'Iwasawa de $\Gamma$. Profitons-en pour remarquer que le caractère cyclotomique $\chi$ envoie $\Gamma$ dans $\mathbb{Z}_{p}^{\times}$et que cette application est un isomorphisme si $K$ est non-ramifié sur $\mathbb{Q}_{p}$.

L'objet de ce chapitre est de donner quelques rappels, sur la théorie de Hodge $p$-adique, la théorie des $(\varphi, \Gamma)$-modules, la cohomologie galoisienne et l'exponentielle de Bloch-Kato.

1.1. Théorie de Hodge $\boldsymbol{p}$-adique. Dans ce paragraphe, on rappelle quelques unes des constructions de Fontaine (voir [Fon94a], [Fon94b]) qui sont utilisées dans la suite de cet article. On note $\sigma$ le Frobenius arithmétique absolu agissant sur $\mathbb{Q}_{p}^{\text {nr }}$.

Soient $\boldsymbol{B}_{\text {cris }}, \boldsymbol{B}_{\mathrm{st}}$ et $\boldsymbol{B}_{\mathrm{dR}}$ les anneaux de périodes $p$-adiques construits par Fontaine (voir [Fon94a] par exemple). Le corps $\boldsymbol{B}_{\mathrm{dR}}=\boldsymbol{B}_{\mathrm{dR}}^{+}[1 / t]$ est une $\mathbb{Q}_{p}$-algèbre qui contient $\overline{\mathbb{Q}}_{p}$ et qui est munie d'une action de $G_{K}$ ainsi que d'une filtration décroissante exhaustive et séparée par des Fil ${ }^{i} \boldsymbol{B}_{\mathrm{dR}}=t^{i} \boldsymbol{B}_{\mathrm{dR}}^{+}$. Remarquons que l'uniformisante $t=\log [\varepsilon]$ dépend du choix de $\varepsilon=\left(\zeta_{p^{n}}\right)_{n \geqslant 0}$ que l'on a fait ci-dessus. L'anneau $\boldsymbol{B}_{\text {st }}$ est une $\mathbb{Q}_{p}$-algèbre qui contient $\widehat{\mathbb{Q}}_{p}^{\text {nr }}$ et qui est munie d'une action de $G_{K}$ ainsi que d'un endomorphisme $\varphi$ commutant à l'action de $G_{K}$ et $\sigma$-semi-linéaire et d'un opérateur de monodromie $N: \boldsymbol{B}_{\text {st }} \rightarrow \boldsymbol{B}_{\text {st }}$ qui commute à l'action de $G_{K}$ et vérifie $N \circ \varphi=p \varphi \circ N$. Enfin, $\boldsymbol{B}_{\text {cris }}=\boldsymbol{B}_{\text {st }}^{N=0}$. On a donc $\boldsymbol{B}_{\text {cris }} \subset \boldsymbol{B}_{\text {st }}$ et de plus on a une injection $\overline{\mathbb{Q}}_{p} \otimes_{\mathbb{Q}_{p}^{\text {nr }}} \boldsymbol{B}_{\mathrm{st}} \hookrightarrow \boldsymbol{B}_{\mathrm{dR}}$.

Pour toute représentation $p$-adique $V$ de $G_{K}$, on pose $\boldsymbol{D}_{\mathrm{dR}}^{K}(V)=\left(\boldsymbol{B}_{\mathrm{dR}} \otimes_{\mathbb{Q}_{p}} V\right)^{G_{K}}$, ce qui fait que $\boldsymbol{D}_{\mathrm{dR}}^{K}(V)$ est un $K$-espace vectoriel filtré de dimension finie. S'il n'y a pas de confusion possible quant au corps $K$, on écrit plus simplement $\boldsymbol{D}_{\mathrm{dR}}(V)$. De manière analogue on pose :

$$
\boldsymbol{D}_{\text {cris }}^{K}(V)=\left(\boldsymbol{B}_{\text {cris }} \otimes_{\mathbb{Q}_{p}} V\right)^{G_{K}} \quad \text { et } \quad \boldsymbol{D}_{\mathrm{pst}}(V)=\underset{L / K}{\underset{\lim }{\longrightarrow}}\left(\boldsymbol{B}_{\mathrm{st}} \otimes_{\mathbb{Q}_{p}} V\right)^{G_{L}},
$$

où $L$ parcourt l'ensemble des extensions finies de $K$, ce qui fait de $D_{\text {cris }}^{K}(V)$ un $K_{0^{-}}$ espace vectoriel muni d'une action $\sigma$-semi-linéaire de $\varphi$ et de $\boldsymbol{D}_{\mathrm{pst}}(V)$ un $K_{0}^{\mathrm{nr}}$-espace vectoriel muni des opérateurs $\varphi$ et $N$ vérifiants $N \circ \varphi=p \varphi \circ N$. Comme ci-dessus, on écrit $\boldsymbol{D}_{\text {cris }}(V)$ s'il n'y a pas de confusion possible. On a :

$$
\operatorname{dim}_{K_{0}} \boldsymbol{D}_{\text {cris }}^{K}(V) \leqslant \operatorname{dim}_{K_{0}^{\mathrm{nr}}} \boldsymbol{D}_{\mathrm{pst}}(V) \leqslant \operatorname{dim}_{K} \boldsymbol{D}_{\mathrm{dR}}^{K}(V) \leqslant \operatorname{dim}_{\mathbb{Q}_{p}} V .
$$


On dit que la représentation $V$ est cristalline (resp. potentiellement semi-stable, resp. de de Rham) si $\operatorname{dim}_{K_{0}} \boldsymbol{D}_{\text {cris }}^{K}(V)=\operatorname{dim}_{\mathbb{Q}_{p}} V$ (resp. si $\operatorname{dim}_{K_{0}^{\text {nr }}} \boldsymbol{D}_{\text {pst }}(V)=\operatorname{dim}_{\mathbb{Q}_{p}} V$, resp. si $\left.\operatorname{dim}_{K} \boldsymbol{D}_{\mathrm{dR}}^{K}(V)=\operatorname{dim}_{\mathbb{Q}_{p}} V\right)$.

Si $V$ est une représentation de de Rham, on pose :

$$
h_{i}(V)=\operatorname{dim}_{K}\left(\operatorname{Fil}^{i} \boldsymbol{D}_{\mathrm{dR}}^{K}(V) / \mathrm{Fil}^{i+1} \boldsymbol{D}_{\mathrm{dR}}^{K}(V)\right) .
$$

La décomposition de Hodge-Tate de $V$ s'écrit alors $\mathbb{C}_{p} \otimes_{\mathbb{Q}_{p}} V \simeq \bigoplus_{i \in \mathbb{Z}} \mathbb{C}_{p}(-i)^{h_{i}(V)}$ où $\mathbb{C}_{p}$ est le complété $p$-adique de $\overline{\mathbb{Q}}_{p}$. Les opposés des entiers $i$ tels que $h_{i}(V) \neq 0$ sont les poids de Hodge-Tate de $V$. On pose $t_{H}(V)=\sum_{i \in \mathbb{Z}} i h_{i}(V)$.

1.2. Modules de Wach et $(\varphi, \Gamma)$-modules. Soit $K / \mathbb{Q}_{p}$ une extension finie que l'on suppose ici non-ramifiée. On note $\boldsymbol{E}^{+}=\lim \left(\mathcal{O}_{\overline{\mathbb{Q}}_{p}} / p \mathcal{O}_{\overline{\mathbb{Q}}_{p}}\right)$ l'anneau construit par Fontaine (voir [Fon91] par exemple, cet anneau s'y appelle $\mathcal{R}$ ), $\boldsymbol{E}=\operatorname{Frac}\left(\boldsymbol{E}^{+}\right)$son corps des fractions et $W(\boldsymbol{E})$ l'anneau des vecteurs de Witt à coefficients dans $\boldsymbol{E}$. On pose $X=[\varepsilon]-1$, avec $\varepsilon=\left(\zeta_{p^{n}}\right)_{n \geqslant 0}, \boldsymbol{A}_{K}^{+}=\mathcal{O}_{K}[[X]]$ et on note $\boldsymbol{A}_{K}$ le complété $p$-adique de $\boldsymbol{A}_{K}^{+}[1 / X]$. Les anneaux $\boldsymbol{A}_{K}^{+}$et $\boldsymbol{A}_{K}$ sont munis d'un Frobenius $\varphi$ et d'une action de $\Gamma=\operatorname{Gal}\left(K_{\infty} / K\right)$, donnés par les formules $\varphi(X)=(1+X)^{p}-1$ et $\gamma(X)=(1+X)^{\chi(\gamma)}-1$ pour $\gamma \in \Gamma$, où $\chi: \Gamma \rightarrow \mathbb{Z}_{p}^{\times}$est le caractère cyclotomique. Soit $\boldsymbol{B}$ le complété $p$-adique de l'extension maximale non-ramifiée du corps $\boldsymbol{B}_{K}=$ $\mathbb{Q}_{p} \otimes_{\mathbb{Z}_{p}} \boldsymbol{A}_{K}$ dans $W(\boldsymbol{E})[1 / p]$. On pose $\boldsymbol{A}=\boldsymbol{B} \cap W(\boldsymbol{E}), \boldsymbol{B}^{+}=\boldsymbol{B} \cap W\left(\boldsymbol{E}^{+}\right)[1 / p]$ et $\boldsymbol{A}^{+}=\boldsymbol{A} \cap W\left(\boldsymbol{E}^{+}\right)$. Tous ces anneaux sont munis d'une action de $G_{K}$ et d'un Frobenius $\varphi$. Enfin, on a $\boldsymbol{A}_{K}=\boldsymbol{A}^{H_{K}}$.

Un $(\varphi, \Gamma)$-module est un module libre de rang fini sur $\boldsymbol{A}_{K}$ muni d'un Frobenius semi-linéaire $\varphi$ et d'une action continue et semi-linéaire de $\Gamma$ commutant avec $\varphi$. Dans [Fon91], Fontaine a défini un foncteur :

$$
\boldsymbol{D}: T \mapsto \boldsymbol{D}(T)=\left(\boldsymbol{A} \otimes_{\mathbb{Z}_{p}} T\right)^{H_{K}},
$$

qui fournit une équivalence entre la catégorie des $\mathbb{Z}_{p}$-représentations de $G_{K}$ et la catégorie des $(\varphi, \Gamma)$-modules étales. Le foncteur :

$$
M \mapsto\left(\boldsymbol{A} \otimes_{A_{K}} M\right)^{\varphi=1}
$$

est un quasi-inverse de $\boldsymbol{D}$. De même, le foncteur $\boldsymbol{D}: V \mapsto\left(\boldsymbol{B} \otimes_{\mathbb{Q}_{p}} V\right)^{H_{K}}$ donne une équivalence entre la catégorie des représentations $p$-adiques de $G_{K}$ et la catégorie des $(\varphi, \Gamma)$-modules étales sur $\boldsymbol{B}_{K}=\mathbb{Q}_{p} \otimes_{\mathbb{Z}_{p}} \boldsymbol{A}_{K}$.

Si $V$ est une représentation cristalline et $T$ un réseau de $V$ stable sous l'action de $G_{K}$, alors un résultat de Colmez [Col99a] dit qu'il existe une base de $\boldsymbol{D}(T)$ dans laquelle les matrices de $\varphi$ et de $\gamma \in \Gamma$ sont à coefficients dans $\boldsymbol{A}_{K}^{+}$. Plus précisement, on a le résultat suivant (cf. la proposition II.1.1 et le théorème III.3.1 de [Ber04]) : 
Proposition 1.1. Si $V$ est une représentation cristalline dont les opposés des poids de Hodge-Tate sont $0=r_{1} \leqslant r_{2} \leqslant \cdots \leqslant r_{d}=h$, alors il existe un unique sous $\boldsymbol{A}_{K}^{+}$-module $\boldsymbol{N}(T)$ de $\boldsymbol{D}^{+}(T)=\left(\boldsymbol{A}^{+} \otimes_{\mathbb{Z}_{p}} T\right)^{H_{K}}$, stable par $\varphi$ et qui satisfait les conditions suivantes :

(1) $\boldsymbol{N}(T)$ est un $\boldsymbol{A}_{K}^{+}$-module libre de rang $d=\operatorname{dim}(V)$ et contient une base de $\boldsymbol{D}(T)$ sur $\boldsymbol{A}_{K}$

(2) l'action de $\Gamma$ préserve $N(T)$ et elle est triviale sur $N(T) / X N(T)$;

(3) $X^{h} D^{+}(T) \subset N(T)$.

De plus, on a $q^{h} N(T) \subset \varphi^{*} N(T)$, où $q=\varphi(X) / X$ et $\varphi^{*} N(T)$ est le $\boldsymbol{A}_{K}^{+}$-module engendré par $\varphi(N(T))$.

On pose alors $N(V)=B_{K}^{+} \otimes_{A_{K}^{+}} N(T)$ où $B_{K}^{+}=\mathbb{Q}_{p} \otimes_{\mathbb{Z}_{p}} A_{K}^{+}$et cette définition ne dépend pas du choix de $T$.

Soit $\boldsymbol{B}_{\text {rig, } K}^{+}$l'ensemble des séries formelles $f(X)=\sum_{k=0}^{\infty} a_{k} X^{k}$, avec $a_{k} \in K$ et telles que $f(X)$ converge sur le disque unité ouvert $\left\{\left.x \in \mathbb{C}_{p}|| x\right|_{p}<1\right\}$. L'anneau $\boldsymbol{B}_{\text {rig, } K}^{+}$est de Bézout [Laz62] et de plus il admet la théorie des diviseurs élémentaires ; il est aussi muni d'actions de $\varphi$ et de $\Gamma$ et on a un plongement $\varphi^{-n}: \boldsymbol{B}_{\mathrm{rig}, K}^{+} \hookrightarrow$ $K_{n}[[t]] \subset \boldsymbol{B}_{\mathrm{dR}}^{+}$qui envoie $X \operatorname{sur} \zeta_{p^{n}} \exp \left(t / p^{n}\right)-1$.

Proposition 1.2. Si $V$ est une représentation cristalline dont les opposés des poids de Hodge-Tate sont $0=r_{1} \leqslant r_{2} \leqslant \cdots \leqslant r_{d}=h$, alors $\boldsymbol{D}_{\text {cris }}(V) \simeq\left(\boldsymbol{B}_{\mathrm{rig}, K}^{+} \otimes_{\boldsymbol{A}_{K}^{+}}\right.$ $N(T))^{\Gamma}$ et :

$$
\left[\boldsymbol{B}_{\mathrm{rig}, K}^{+} \otimes_{\boldsymbol{A}_{K}^{+}} \boldsymbol{N}(T): \boldsymbol{B}_{\mathrm{rig}, K}^{+} \otimes_{K} \boldsymbol{D}_{\text {cris }}(V)\right]=\left[\left(\frac{t}{X}\right)^{r_{1}} ; \ldots ;\left(\frac{t}{X}\right)^{r_{h}}\right] .
$$

Preuve. Voir [Ber04, proposition III.4].

1.3. Cohomologie galoisienne. Rappelons maintenant comment on peut calculer la cohomologie galoisienne des représentations $p$-adiques à partir des $(\varphi, \Gamma)$-modules. On suppose toujours que $K / \mathbb{Q}_{p}$ est non-ramifiée, on pose $\Gamma_{n}=\mathrm{Gal}\left(K_{\infty} / K_{n}\right)$, on fixe un générateur topologique $\gamma_{1}$ de $\Gamma_{1}$ et on pose $\gamma_{n}=\gamma_{1}^{p^{n-1}}$. Si $T$ est une représentation $\mathbb{Z}_{p}$-adique de $G_{K}$, on note $C_{\varphi, \gamma_{n}}\left(K_{n}, T\right)$ le complexe :

$$
0 \rightarrow \boldsymbol{D}(T) \stackrel{f}{\rightarrow} \boldsymbol{D}(T) \oplus \boldsymbol{D}(T) \stackrel{g}{\rightarrow} \boldsymbol{D}(T) \rightarrow 0,
$$

où les applications $f$ et $g$ sont définies par $f(x)=\left((\varphi-1) x,\left(\gamma_{n}-1\right) x\right)$ et $g(y, z)=$ $\left(\gamma_{n}-1\right) y-(\varphi-1) z$.

Dans [Her98], Herr a montré que les groupes de cohomologie $H^{i}\left(C_{\varphi, \gamma_{n}}\left(K_{n}, T\right)\right)$ s'identifient canoniquement aux groupes de cohomologie galoisienne $H^{i}\left(K_{n}, T\right)$ 
(voir [Ben00, proposition 1.3.2] ou bien [CC99, proposition I.4.1] ou encore [Ber03, proposition I.8] pour une description explicite de cet isomorphisme quand $i=1$ ).

Enfin, on peut aussi retrouver la cohomologie d'Iwasawa :

$$
H_{\mathrm{IW}}^{i}(K, T)=\lim _{\operatorname{cor}_{K_{n} / K_{n-1}}} H^{i}\left(K_{n}, T\right)
$$

en utilisant les $(\varphi, \Gamma)$-modules. Pour cela, on utilise l'opérateur $\psi: \boldsymbol{B} \rightarrow \boldsymbol{B}$ qui est défini par la formule :

$$
\psi(x)=\frac{1}{p} \varphi^{-1}\left(\operatorname{Tr}_{\boldsymbol{B} / \varphi(\boldsymbol{B})}(x)\right) .
$$

L'opérateur $\psi$ commute à l'action de $G_{K}$ et on a $\psi \circ \varphi=$ id. La cohomologie du complexe :

$$
\boldsymbol{D}(T) \stackrel{\psi-1}{\longrightarrow} \boldsymbol{D}(T)
$$

s'identifie canoniquement à la cohomologie d'Iwasawa de $T$, c'est-à-dire que $H_{\mathrm{IW}}^{1}(K, T) \simeq \boldsymbol{D}(T)^{\psi=1}$ et que $H_{\mathrm{Iw}}^{2}(K, T) \simeq \boldsymbol{D}(T) /(\psi-1)$ (voir [CC99, §II.3]). Donnons une description explicite du premier isomorphisme. Si $\alpha \in \boldsymbol{D}(T)^{\psi=1}$, alors $(\varphi-1) \alpha \in \boldsymbol{D}(T)^{\psi=0}$ et comme $\gamma_{n}-1$ est inversible sur $\boldsymbol{D}(T)^{\psi=0}$ (cf. [Her98] ou [CC99, proposition I.5.1]), il existe $x_{n} \in \boldsymbol{D}(T)$ vérifiant $\left(\gamma_{n}-1\right) x_{n}=(\varphi-1) \alpha$. Les $\mathrm{cl}\left(x_{n}, \alpha\right) \in H^{1}\left(C_{\varphi, \gamma_{n}}\left(K_{n}, T\right)\right)$ forment alors un système compatible d'éléments de $H^{1}\left(K_{n}, T\right)$.

1.4. L'exponentielle de Bloch-Kato. Dans cette section, $K$ désigne une extension finie quelconque de $\mathbb{Q}_{p}$. On note $K_{0}$ la sous-extension non ramifiée maximale de $K$. Soit $V$ une représentation de de Rham de $G_{K}$. Bloch et Kato ont défini (voir [BK90, §4]) la partie exponentielle (resp. parties finie et géométrique) de $H^{1}(K, V)$ en posant :

$$
\begin{aligned}
& H_{e}^{1}(K, V)=\operatorname{ker}\left(H^{1}(K, V) \rightarrow H^{1}\left(K, \boldsymbol{B}_{\mathrm{cris}}^{\varphi=1} \otimes_{\mathbb{Q}_{p}} V\right)\right), \\
& H_{f}^{1}(K, V)=\operatorname{ker}\left(H^{1}(K, V) \rightarrow H^{1}\left(K, \boldsymbol{B}_{\mathrm{cris}} \otimes_{\mathbb{Q}_{p}} V\right)\right), \\
& H_{g}^{1}(K, V)=\operatorname{ker}\left(H^{1}(K, V) \rightarrow H^{1}\left(K, \boldsymbol{B}_{\mathrm{dR}} \otimes_{\mathbb{Q}_{p}} V\right)\right) .
\end{aligned}
$$

La dualité locale fournit un accouplement $(\cdot, \cdot)_{V}: H^{1}(K, V) \times H^{1}\left(K, V^{*}(1)\right) \rightarrow$ $\mathbb{Q}_{p}$ pour lequel l'orthogonal de $H_{e}^{1}(K, V)$ est $H_{g}^{1}\left(K, V^{*}(1)\right)$ et celui de $H_{f}^{1}(K, V)$ est $H_{f}^{1}\left(K, V^{*}(1)\right)$. L'espace tangent de $V$ sur $K$ est par définition le quotient :

$$
t_{V}(K)=\boldsymbol{D}_{\mathrm{dR}}^{K}(V) / \operatorname{Fil}^{0} \boldsymbol{D}_{\mathrm{dR}}^{K}(V) .
$$

Les anneaux $\boldsymbol{B}_{\text {cris }}$ et $\boldsymbol{B}_{\mathrm{dR}}$ sont reliés par l'inclusion $\boldsymbol{B}_{\text {cris }} \subset \boldsymbol{B}_{\mathrm{dR}}$ mais aussi et 
surtout par les suites exactes fondamentales :

$$
\begin{array}{r}
0 \rightarrow \mathbb{Q}_{p} \rightarrow \boldsymbol{B}_{\text {cris }}^{\varphi=1} \stackrel{\alpha}{\rightarrow} \boldsymbol{B}_{\mathrm{dR}} / \mathrm{Fil}^{0} \boldsymbol{B}_{\mathrm{dR}} \rightarrow 0, \\
0 \rightarrow \mathbb{Q}_{p} \rightarrow \boldsymbol{B}_{\text {cris }} \stackrel{\beta}{\rightarrow} \boldsymbol{B}_{\text {cris }} \oplus \boldsymbol{B}_{\mathrm{dR}} / \mathrm{Fil}^{0} \boldsymbol{B}_{\mathrm{dR}} \rightarrow 0,
\end{array}
$$

où $\alpha(x)=x \bmod \mathrm{Fil}^{0} \boldsymbol{B}_{\mathrm{dR}}$ et $\beta(x)=\left((1-\varphi) x, x \bmod \mathrm{Fil}^{0} \boldsymbol{B}_{\mathrm{dR}}\right)$. En prenant les produits tensoriels de ces suites par $V$ et les invariants sous l'action de $G_{K}$, on obtient des suites exactes longues de cohomologie qui nous donnent les deux suites exactes :

$$
\begin{gathered}
0 \rightarrow H^{0}(K, V) \rightarrow \boldsymbol{D}_{\text {cris }}(V)^{\varphi=1} \rightarrow t_{V}(K) \rightarrow H_{e}^{1}(K, V) \rightarrow 0, \\
0 \rightarrow H^{0}(K, V) \rightarrow \boldsymbol{D}_{\text {cris }}(V) \rightarrow \boldsymbol{D}_{\text {cris }}(V) \oplus t_{V}(K) \rightarrow H_{f}^{1}(K, V) \rightarrow 0 .
\end{gathered}
$$

L'application de connexion $\exp _{V, K}: t_{V}(K) \rightarrow H^{1}(K, V)$ dans la première suite s'appelle l'exponentielle de Bloch et Kato. On définit l'exponentielle duale $\exp _{V, K}^{*}: H^{1}(K, V) \rightarrow \operatorname{Fil}^{0} D_{\mathrm{dR}}^{K}(V)$ par la formule :

$$
\operatorname{Tr}_{K / \mathbb{Q}_{p}}\left[\exp _{V, K}^{*}(x), y\right]_{V}=\left(x, \exp _{V^{*}(1), K}(y)\right)_{V}
$$

où $[\cdot, \cdot]_{V}: \boldsymbol{D}_{\mathrm{dR}}^{K}(V) \times \boldsymbol{D}_{\mathrm{dR}}^{K}\left(V^{*}(1)\right) \rightarrow K$ est la dualité canonique. On vérifie facilement que $\operatorname{ker}\left(\exp _{V, K}^{*}\right)=H_{g}^{1}(K, V)$.

Lemme 1.3. On a des isomorphismes canoniques :

$$
\begin{aligned}
\exp _{V, f / e}: & \frac{\boldsymbol{D}_{\text {cris }}(V)}{(1-\varphi) \boldsymbol{D}_{\text {cris }}(V)} \stackrel{\sim}{\rightarrow} \frac{H_{f}^{1}(K, V)}{H_{e}^{1}(K, V)}, \\
\exp _{V, g / f}^{*}: & \frac{H_{g}^{1}(K, V)}{H_{f}^{1}(K, V)} \stackrel{\sim}{\rightarrow} \boldsymbol{D}_{\text {cris }}(V)^{\varphi=p^{-1}}
\end{aligned}
$$

Avant de montrer ce lemme, montrons un lemme technique qui est sans-doute bien connu.

Lemme 1.4. Si $W$ est un $K_{0}$-espace vectoriel de dimension finie muni d'un isomorphisme $\sigma$-semi-linéaire $\varphi$, alors l'application qui à tout $f \in \operatorname{Hom}_{K_{0}}\left(W, K_{0}\right)^{\varphi=1}$ associe $\operatorname{Tr}_{K_{0} / \mathbb{Q}_{p}}(f)$ fournit un isomorphisme :

$$
\operatorname{Hom}_{K_{0}}\left(W, K_{0}\right)^{\varphi=1} \stackrel{\sim}{\rightarrow} \operatorname{Hom}_{\mathbb{Q}_{p}}\left(W /(1-\varphi) W, \mathbb{Q}_{p}\right) .
$$


Preuve. La forme bilinéaire «trace» de $K_{0} \times K_{0}$ dans $\mathbb{Q}_{p}$ qui à $(x, y)$ associe $\operatorname{Tr}_{K_{0} / \mathbb{Q}_{p}}(x y)$ induit un isomorphisme $K_{0} \simeq \operatorname{Hom}_{\mathbb{Q}_{p}}\left(K_{0}, \mathbb{Q}_{p}\right)$. On en déduit que l'application :

$$
\begin{aligned}
\operatorname{Hom}_{K_{0}}\left(W, K_{0}\right) & \rightarrow \operatorname{Hom}_{\mathbb{Q}_{p}}\left(W, \mathbb{Q}_{p}\right) \\
f & \mapsto \operatorname{Tr}_{K_{0} / \mathbb{Q}_{p}}(f)
\end{aligned}
$$

est un isomorphisme de $\mathbb{Q}_{p}$-espaces vectoriels. De plus, elle est compatible avec l'action de $\varphi$ car :

$\operatorname{Tr}_{K_{0} / \mathbb{Q}_{p}}(\varphi f)(x)=\operatorname{Tr}_{K_{0} / \mathbb{Q}_{p}}\left(\varphi f\left(\varphi^{-1} x\right)\right)=\operatorname{Tr}_{K_{0} / \mathbb{Q}_{p}} f\left(\varphi^{-1}(x)\right)=\left(\varphi\left(\operatorname{Tr}_{K_{0} / \mathbb{Q}_{p}} f\right)\right)(x)$.

Comme $\operatorname{Hom}_{\mathbb{Q}_{p}}\left(W, \mathbb{Q}_{p}\right)^{\varphi=1}=\operatorname{Hom}_{\mathbb{Q}_{p}}\left(W /(1-\varphi) W, \mathbb{Q}_{p}\right)$, on en déduit le lemme.

Preuve du lemme 1.3. On remarque que la suite (1.1) s'injecte dans (1.2), d'où on obtient le premier isomorphisme. D'autre part, en utilisant la dualité locale et le lemme 1.4, on obtient :

$$
\frac{H_{g}^{1}(K, V)}{H_{f}^{1}(K, V)} \simeq\left(\frac{H_{f}^{1}\left(K, V^{*}(1)\right)}{H_{e}^{1}\left(K, V^{*}(1)\right)}\right)^{*} \simeq\left(\frac{\boldsymbol{D}_{\text {cris }}\left(V^{*}(1)\right)}{(1-\varphi) \boldsymbol{D}_{\text {cris }}\left(V^{*}(1)\right)}\right)^{*} \stackrel{\sim}{\rightarrow} \boldsymbol{D}_{\text {cris }}(V)^{\varphi=p^{-1}},
$$

et le lemme est démontré.

On pose maintenant $L_{f}(K, V)=\operatorname{det}_{\mathbb{Q}_{p}} H^{0}(K, V) \otimes_{\mathbb{Q}_{p}} \operatorname{det}_{\mathbb{Q}_{p}}^{-1} H_{f}^{1}(K, V)$. La suite exacte (1.2) fournit alors un isomorphisme canonique $i_{V}: L_{f}(K, V) \simeq \operatorname{det}_{\mathbb{Q}_{p}}^{-1} t_{V}(K)$.

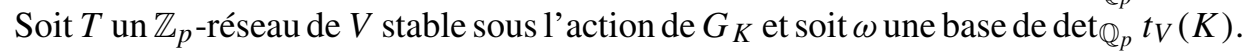
On note $H_{f}^{1}(K, T)$ l'image inverse de $H_{f}^{1}(K, V)$ dans $H^{1}(K, T)$ et l'on pose :

$$
L_{f}(K, T)=\operatorname{det}_{\mathbb{Z}_{p}} H^{0}(K, T) \otimes_{\mathbb{Z}_{p}} \operatorname{det}_{\mathbb{Z}_{p}}^{-1} H_{f}^{1}(K, T) .
$$

Lemme 1.5. On a des suites exactes :

$$
\begin{aligned}
0 \rightarrow & \frac{H^{1}(K, V)}{H_{f}^{1}(K, V)} \rightarrow \boldsymbol{D}_{\text {cris }}\left(V^{*}(1)\right)^{*} \oplus t_{V^{*}(1)}^{*}(K) \\
& \rightarrow \boldsymbol{D}_{\text {cris }}\left(V^{*}(1)\right)^{*} \rightarrow H^{2}(K, V) \rightarrow 0, \\
0 \rightarrow & \frac{H^{1}(K, V)}{H_{g}^{1}(K, V)} \rightarrow \operatorname{Fil}^{0} \boldsymbol{D}_{\mathrm{dR}}^{K}(V) \\
\rightarrow & \frac{\boldsymbol{D}_{\text {cris }}(V)}{\left(1-p^{-1} \varphi^{-1}\right) \boldsymbol{D}_{\text {cris }}(V)} \rightarrow\left(V^{*}(1)^{G_{K}}\right)^{*} \rightarrow 0
\end{aligned}
$$


Preuve. Il suffit de dualiser les suites exactes (1.1) et (1.2) en remplaçant $V$ par $V^{*}(1)$ et d'utiliser la dualité locale et les isomorphismes $t_{V^{*}(1)}^{*}(K) \simeq \operatorname{Fil}^{0} D_{\mathrm{dR}}^{K}(V)$ et $\left(\boldsymbol{D}_{\text {cris }}\left(V^{*}(1)\right)^{\varphi=1}\right)^{*} \simeq \boldsymbol{D}_{\text {cris }}(V) /\left(1-p^{-1} \varphi^{-1}\right) \boldsymbol{D}_{\text {cris }}(V)$.

Définition 1.6. On appelle nombre de Tamagawa, et on note $\operatorname{Tam}_{K, \omega}^{0}(T)$, l'unique puissance de $p$ telle que $i_{V}\left(L_{f}(K, T)\right)=\mathbb{Z}_{p} \operatorname{Tam}_{K, \omega}^{0}(T) \omega^{-1}$, où $\omega^{-1}$ est la base duale de $\omega$ (voir [BK90], [Per95]).

Ces nombres interviennent dans la formulation de la conjecture $C_{\mathrm{EP}}(K, V)$ (conjecture 2.20 ci-dessous).

\section{Déterminants et constantes locales}

L'objet de ce chapitre est d'énoncer la conjecture $C_{\mathrm{EP}}(L / K, V)$. On commence par des rappels sur la théorie des déterminants généralisés, puis on passe en revue la construction des constantes locales, pour les représentations de Weil-Deligne tout d'abord, et pour les représentations potentiellement semi-stables ensuite.

2.1. Déterminants généralisés. Dans le reste de cet article, nous avons besoin de la construction de déterminants sur des anneaux tels que $\mathbb{Z}_{p}[G]$ ou $\mathbb{Q}_{p}[G]$, pour un groupe abélien fini $G$, ou encore $\mathbb{Z}_{p}[[X]]$ et $\mathbb{Q}_{p} \otimes_{\mathbb{Z}_{p}} \mathbb{Z}_{p}[[X]]$. Nous commençons donc par quelques rappels, tirés de [KM76], [De187], [BF01], sur le formalisme très général des déterminants.

Soit $A$ un anneau commutatif unitaire. On note $\boldsymbol{M}(A)$ la catégorie des $A$-modules et $\boldsymbol{P}(A)$ la sous-catégorie de $\boldsymbol{M}(A)$ formée des modules projectifs de type fini.

On appelle catégorie de Picard une catégorie $\mathcal{P}$ dont toute flèche est un isomorphisme, munie d'un foncteur $\nabla: \mathcal{P} \times \mathcal{P} \rightarrow \mathcal{P}$ et d'une contrainte d'associativité pour $\otimes$. On peut déduire de ces axiomes l'existence d'un objet unité $\mathbf{1}_{\mathcal{P}}$, unique à isomorphisme près. Tout objet $X$ de $\mathcal{P}$ admet un inverse $X^{-1}$ tel que $X \otimes X^{-1} \simeq \mathbf{1}_{\mathcal{P}}$. On dit qu'une catégorie de Picard $\mathcal{P}$ est commutative si elle est munie d'une contrainte de commutativité compatible à la contrainte d'associativité.

Soit $(\boldsymbol{P}(A)$, is) la catégorie dont les objets sont ceux de $\boldsymbol{P}(A)$ et dont les flèches sont les isomorphismes. On appelle foncteur déterminant un foncteur det: $(\boldsymbol{P}(A)$, is $) \rightarrow \mathcal{P}$ vérifiant les propriétés suivantes :

(1) Pour toute suite exacte $0 \rightarrow P^{\prime} \rightarrow P \rightarrow P^{\prime \prime} \rightarrow 0$, on a un isomorphisme fonctoriel : $\operatorname{det}(P) \simeq \operatorname{det}\left(P^{\prime}\right) \otimes \operatorname{det}\left(P^{\prime \prime}\right)$.

(2) Pour toute suite exacte $0 \rightarrow P \stackrel{\alpha}{\rightarrow} Q \rightarrow 0$, l'application $\operatorname{det}(\alpha)$ coïncide avec le composé :

$$
\operatorname{det}(P) \simeq \operatorname{det}(0) \otimes \operatorname{det}(Q) \simeq \operatorname{det}(Q),
$$


et de même $\operatorname{det}(\alpha)^{-1}$ coïncide avec le composé :

$$
\operatorname{det}(Q) \simeq \operatorname{det}(P) \otimes \operatorname{det}(0) \simeq \operatorname{det}(P) .
$$

(3) Si $P=P^{\prime} \oplus P^{\prime \prime}$ et si : $0 \rightarrow P^{\prime} \rightarrow P \rightarrow P^{\prime \prime} \rightarrow 0$ et $0 \rightarrow P^{\prime \prime} \rightarrow P \rightarrow P^{\prime} \rightarrow 0$ sont les suites exactes naturelles, alors le diagramme :

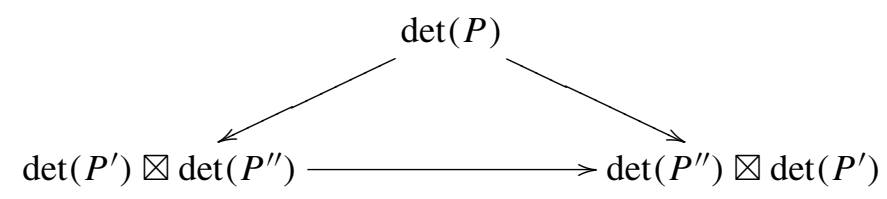

est commutatif.

(4) Pour tout module projectif $P$ muni d'une filtration $P \supset P^{\prime} \supset P^{\prime \prime} \supset\{0\}$, le diagramme :

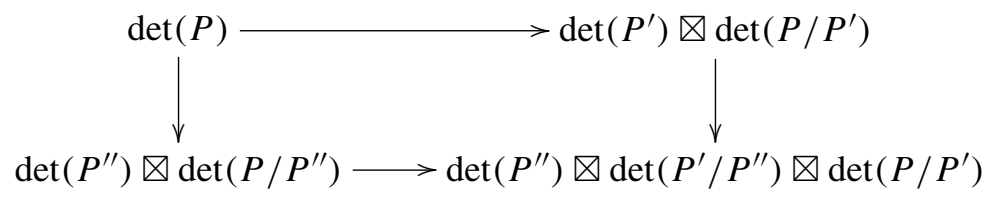

est commutatif.

Soit $\boldsymbol{K}(A)=\boldsymbol{K}(\boldsymbol{M}(A))$ la catégorie des complexes de $A$-modules. On dit qu'un morphisme de complexes $f: M^{\bullet} \rightarrow N^{\bullet}$ est un quasi-isomorphisme si pour tout $i$, l'application $H^{i}\left(M^{\bullet}\right) \rightarrow H^{i}\left(N^{\bullet}\right)$ est un isomorphisme. La catégorie dérivée $\boldsymbol{D}(A)=$ $\boldsymbol{D}(\boldsymbol{K}(A))$ est la localisation de $\boldsymbol{K}(A)$ par rapport aux quasi-isomorphismes.

On dit qu'un objet $M^{\bullet}$ de $\boldsymbol{D}(A)$ est parfait s'il existe un complexe borné de $A$ modules projectifs de type fini : $P^{\bullet}=\left(\cdots \rightarrow P_{i+1} \rightarrow P_{i} \rightarrow P_{i-1} \rightarrow \cdots\right)$ quasiisomorphe à $M^{\bullet}$. Soit $D^{\mathrm{p}}(A)$ la sous-catégorie de $\boldsymbol{D}(A)$ formée des objets parfaits. Pour tout objet $M^{\bullet}$ de $D^{\mathrm{p}}(A)$, on fixe un complexe $P^{\bullet}$ vérifiant les conditions cidessus et l'on pose :

$$
\operatorname{det}\left(M^{\bullet}\right)=\bigotimes_{i \in \mathbb{Z}} \operatorname{det}\left(P_{i}\right)^{(-1)^{i}}
$$

On obtient ainsi une extension du foncteur det, unique à équivalence près, à un foncteur (encore noté det) :

$$
\operatorname{det}:\left(D^{\mathrm{p}}(A), \text { qis }\right) \rightarrow \mathcal{P} .
$$

Si les modules de cohomologie $H^{i}\left(M^{\bullet}\right)$ sont parfaits en toutes dimensions, on a alors un isomorphisme fonctoriel :

$$
\operatorname{det}\left(M^{\bullet}\right) \simeq \bigotimes_{i \in \mathbb{Z}} \operatorname{det}\left(H^{i}\left(M^{\bullet}\right)\right)^{(-1)^{i}}
$$


Dans [De187], Deligne construit une catégorie de Picard commutative $\mathcal{V}(A)$ et un foncteur déterminant universel $[\cdot]_{A}:(\boldsymbol{P}(A)$, is $) \rightarrow \mathcal{V}(A)$ tel que tout foncteur déterminant det s'écrit comme le composé de $[\cdot]_{A}$ avec un foncteur additif $\mathcal{V}(A) \rightarrow \mathcal{P}$. On en déduit en particulier un foncteur $[\cdot]_{A}:\left(D^{\mathrm{p}}(A)\right.$, qis $) \rightarrow \mathcal{V}(A)$. La proposition ci-dessous rassemble quelques propriétés du foncteur $[\cdot]_{A}$.

Proposition 2.1. Si $f: A \rightarrow B$ est un morphisme d'anneaux, alors le foncteur « extension des scalaires » $f^{*} M=B \otimes_{A} M$ induit des foncteurs $\boldsymbol{L} f^{*}: \boldsymbol{D}^{\mathrm{p}}(A) \rightarrow D^{\mathrm{p}}(B)$ et $f^{*}: \mathcal{V}(A) \rightarrow \mathcal{V}(B)$, et lesfoncteurs $[\cdot]_{B} \circ \boldsymbol{L} f^{*}$ et $f^{*} \circ[\cdot]_{A}:\left(D^{\mathrm{p}}(A)\right.$, qis $) \rightarrow \mathcal{V}(B)$ sont quasi-isomorphes.

Si on suppose de plus que $B$ est projectif de type fini sur A, alors la restriction des scalaires induit des foncteurs $f_{*}: D^{\mathrm{p}}(B) \rightarrow D^{\mathrm{p}}(A)$ et $f_{*}: \mathcal{V}(B) \rightarrow \mathcal{V}(A)$, et les foncteurs $f_{*} \circ[\cdot]_{B}$ et $[\cdot]_{A} \circ f_{*}:\left(D^{\mathrm{p}}(B)\right.$, qis $) \rightarrow \mathcal{V}(A)$ sont quasi-isomorphes.

Preuve. Voir [Del87, section 4.11].

On note $\mathcal{P}(A)$ la catégorie des $A$-modules inversibles gradués. Un objet de $\mathcal{P}(A)$ s'identifie à une paire $(X, \alpha)$ où $X$ est un $A$-module inversible et $\alpha: \operatorname{Spec}(A) \rightarrow \mathbb{Z}$ est une fonction localement constante. Une flèche $f:(X, \alpha) \rightarrow(Y, \beta)$ n'existe que si $\alpha=\beta$, auquel cas c'est un isomorphisme. On munit $\mathcal{P}(A)$ d'un produit tensoriel en posant

$$
(X, \alpha) \otimes(Y, \beta)=\left(X \otimes_{A} Y, \alpha+\beta\right) .
$$

Munie de la contrainte de commutativité donnée par la règle de Koszul :

$$
\begin{aligned}
\psi: X \otimes_{A} Y & \rightarrow Y \otimes_{A} X \\
\psi(x \otimes y) & =(-1)^{\alpha \beta} y \otimes x,
\end{aligned}
$$

la catégorie $\mathcal{P}(A)$ est alors une catégorie de Picard commutative. On identifie l'opposé $(X, \alpha)^{-1}$ d'un élément $(X, \alpha)$ à $\left(X^{*},-\alpha\right)$ où $X^{*}=\operatorname{Hom}_{A}(X, A)$.

Si $P$ est un $A$-module projectif de type fini, alors le rang de $P$ est une fonction localement constante $\operatorname{rg}_{P}: \operatorname{Spec}(A) \rightarrow \mathbb{Z}$ et on définit le déterminant de KnudsenMumford $\operatorname{det}_{A}(P)$ en posant :

$$
\operatorname{det}_{A}(P)=\left(\wedge^{r} P, \operatorname{rg}_{P}\right) \in \mathrm{Ob}(\mathcal{P}(A)) .
$$

Remarquons que la propriété universelle du foncteur $[\cdot]_{A}$ donne un foncteur additif $\mathcal{V}(A) \rightarrow \mathcal{P}(A)$ qui n'est pas, en général, une équivalence de catégories.

Dans cet article nous n'utilisons que les déterminants sur des produits finis d'anneaux locaux. Dans ce cas les catégories $\mathcal{V}(A)$ et $\mathcal{P}(A)$ sont équivalentes par [Del87, section 4.13] et la construction de Knudsen-Mumford fournit donc un foncteur déterminant universel. Les anneaux typiques auquels nous allons appliquer la théorie précedente sont : 
(1) $A=\mathbb{Q}_{p}[G]$ ou bien $A=\mathbb{Z}_{p}[G]$, où $G$ est un groupe abélien fini.

(2) $A=\mathbb{Z}_{p}[[X]]$ ou bien $A=\mathbb{Q}_{p} \otimes_{\mathbb{Z}_{p}} \mathbb{Z}_{p}[[X]]$.

Proposition 2.2. Si A est un anneau local régulier de dimension $n$, alors :

(1) Tout A-module de type fini $M$ admet une résolution projective $P^{\bullet}: 0 \rightarrow P_{m} \rightarrow$ $\cdots \rightarrow P_{0} \rightarrow M \rightarrow 0$ avec $m \leqslant n$.

(2) Si $Q(A)$ est l'anneau total des fractions de A, et si $M$ est un A-module de torsion, alors le produit tensoriel de $P^{\bullet}$ par $Q(A)$ donne une suite exacte $0 \rightarrow$ $Q(A) \otimes_{A} P_{m} \rightarrow \cdots \rightarrow Q(A) \otimes_{A} P_{0} \rightarrow 0$. On en déduit une injection canonique $i_{A}: \operatorname{det}_{A}(M) \rightarrow \operatorname{det}_{Q(A)}\left(Q(A) \otimes_{A} P^{\bullet}\right) \simeq Q(A)$ et l'image $\operatorname{de} \operatorname{det}_{A}(M)$ dans $Q(A)$ ne dépend pas du choix de $P^{\bullet}$ et coïncide avec l'idéal fractionnaire de $M$.

Preuve. La première assertion est un théorème classique de Serre (voir par exemple [Mat92, §19]). Pour la deuxième voir [KM76, théorème 3].

Exemple 2.3. En particulier, considérons l'anneau $A=\mathbb{Z}_{p}[[X]]$ qui est local régulier de dimension 2 et soit $M$ un $A$-module de type fini et de torsion. Il existe alors une suite exacte :

$$
0 \rightarrow \text { (fini) } \rightarrow M \rightarrow \bigoplus_{i=1}^{n} A / f_{i} A \rightarrow \text { (fini) } \rightarrow 0,
$$

où les $f_{i}$ sont des polynômes distingués. On a alors $\operatorname{det}_{A}(M)=\operatorname{car}_{A}(M)^{-1} A$ où $\operatorname{car}_{A}(M)=\prod_{i=1}^{n} f_{i}$ est le polynôme caractéristique de $M$.

Remarque 2.4. L'approche de Deligne a été généralisée aux anneaux non-commutatifs par Burns et Flach, voir [BF01].

Pour terminer, rappelons deux lemmes purement techniques qui nous serviront dans la suite. Le lemme suivant sera utilisé dans la preuve du lemme 2.16.

Lemme 2.5. Soient $K / F$ une extension cyclique de degré $f$ et $\sigma$ un générateur de $\operatorname{Gal}(K / F)$. Soient $W$ un $K$-espace vectoriel de dimension finie d et $\alpha: W \rightarrow W$ un isomorphisme $\sigma$-semi-linéaire. On a alors :

$$
\operatorname{det}_{F}(\alpha \mid W)=(-1)^{(f-1) d} \operatorname{det}_{K}\left(\alpha^{f} \mid W\right) .
$$

Preuve. Soit $w_{1}, \ldots, w_{d}$ une base de $W$ sur $K$ et soit $\alpha^{*}: W \rightarrow W$ l'application $K$ linéaire définie par $\alpha^{*}\left(w_{i}\right)=\alpha\left(w_{i}\right)$ pour tout $i=1, \ldots, d$. Si $A_{\alpha}$ est la matrice de $\alpha$ dans la base $\left\{w_{i}\right\}_{i=1, \ldots, d}$, alors la matrice de $\alpha^{f}$ dans la même base est $A A^{\sigma} \ldots A^{\sigma^{f-1}}$, d'où :

$$
\operatorname{det}_{K}\left(\alpha^{f} \mid W\right)=\mathrm{N}_{K / F}(\operatorname{det} A)=\mathrm{N}_{K / F}\left(\operatorname{det}_{K}\left(\alpha^{*} \mid W\right)\right)=\operatorname{det}_{F}\left(\alpha^{*} \mid W\right) .
$$


D'autre part, si $c$ est un générateur de $K$ sur $F[\sigma]$, alors $\left\{\sigma^{j}(c) w_{i}\right\}_{0 \leqslant j \leqslant f-1,1 \leqslant i \leqslant d}$ est une base de $W$ sur $F$. La formule :

$$
\alpha\left(\sigma^{j}(c) w_{i}\right)=\sigma^{j+1}(c) \alpha\left(w_{i}\right)=\alpha^{*}\left(\sigma^{j+1}(c) w_{i}\right)
$$

montre que la matrice de $\alpha$ dans cette base s'obtient en permutant les colonnes de la matrice de $\alpha^{*}$. On en déduit facilement que :

$$
\operatorname{det}_{F}(\alpha \mid W)=(-1)^{(f-1) d} \operatorname{det}_{F}\left(\alpha^{*} \mid W\right)
$$

et donc que :

$$
\operatorname{det}_{F}(\alpha \mid W)=(-1)^{(f-1) d} \operatorname{det}_{K}\left(\alpha^{f} \mid W\right),
$$

ce qui montre le lemme.

Soient maintenant $K$ un corps de caractéristique $0, G$ un groupe abélien fini et $M$ un $K[G]$-module. On note $X(G)$ le groupe des caractères de $G$ et on fixe une extension $E$ de $K$ contenant les valeurs de tous les éléments de $X(G)$. Si $\eta \in X(G)$, on note $e_{\eta}$ l'idempotent habituel :

$$
e_{\eta}=\frac{1}{\sharp G} \sum_{g \in G} \eta^{-1}(g) g .
$$

Soit $M_{E}=E \otimes_{K} M$ et soit $M_{\eta}=e_{\eta}\left(M_{E}\right)=\left\{m \in M_{E} \mid g(m)=\eta(g) m\right.$ pour tout $g \in G\}$. On a alors une décomposition canonique :

$$
\begin{aligned}
M_{E} & \simeq \bigoplus_{\eta \in X(G)} M_{\eta}, \\
x & \mapsto \bigoplus_{\eta \in X(G)} e_{\eta}(x) .
\end{aligned}
$$

Si $M$ est libre de rang fini $r$ sur $K[G]$ et si $\left\{m_{i}\right\}_{i=1, \ldots, r}$ est une base de $M$, alors on pose $\tilde{m}=\wedge_{i=1}^{r} m_{i} \in \operatorname{det}_{K[G]} M$ et $\tilde{m}_{\eta}=\wedge_{i=1}^{r} e_{\eta}\left(m_{i}\right)$. On a alors $\tilde{m}_{\eta}=e_{\eta}(\tilde{m})$ ce qui nous donne un isomorphisme :

$$
\left(\operatorname{det}_{E[G]} M_{E}\right)_{\eta} \simeq \operatorname{det}_{E}\left(M_{\eta}\right) .
$$

Le lemme ci-dessous est alors évident.

Lemme 2.6. Soient $M$ et $N$ deux $K[G]$-modules libres de rang fini et soit :

$$
f: \operatorname{det}_{K[G]} M \otimes_{K[G]} \operatorname{det}_{K[G]}^{-1} N \rightarrow K[G]
$$

un homomorphisme de $K[G]$-modules. Pour tous $\tilde{m} \in \operatorname{det}_{K[G]} M, \tilde{n} \in \operatorname{det}_{K[G]} N$, on a alors :

$$
f\left(\tilde{m} \otimes \tilde{n}^{-1}\right)=\sum_{\eta \in X(G)} f_{\eta}\left(\tilde{m}_{\eta} \otimes \tilde{n}_{\eta}^{-1}\right) e_{\eta},
$$

où $f_{\eta}: \operatorname{det}_{E} M_{\eta} \otimes \operatorname{det}_{E}^{-1} N_{\eta} \rightarrow$ E désigne la $\eta$-composante de $f$. 
2.2. Déterminants de la cohomologie galoisienne. Soit $K$ une extension finie de $\mathbb{Q}_{p}$. On note $\boldsymbol{M}\left(G_{K}\right)$ la catégorie des $\mathbb{Z}_{p}$-représentations de $G_{K}$, c'est-à-dire la catégorie des $\mathbb{Z}_{p}$-modules (pas nécessairement de type fini) munis d'une action linéaire et continue de $G_{K}$. Si $L$ est une extension finie de $K$ on a les foncteurs habituels : $\operatorname{Res}_{L / K}: \boldsymbol{M}\left(G_{K}\right) \rightarrow \boldsymbol{M}\left(G_{L}\right)$ et $\operatorname{Ind}_{L / K}: \boldsymbol{M}\left(G_{L}\right) \rightarrow \boldsymbol{M}\left(G_{K}\right)$, ce dernier foncteur étant donné par la formule $\operatorname{Ind}_{L / K} M=\mathbb{Z}_{p}\left[G_{K}\right] \otimes_{\mathbb{Z}_{p}\left[G_{L}\right]} M$.

Supposons maintenant $L$ abélien sur $K$ et posons $G=\operatorname{Gal}(L / K)$. On note $\iota: \mathbb{Z}_{p}[G] \rightarrow \mathbb{Z}_{p}[G]$ l'involution $g \mapsto g^{-1}$. Si $M \in M\left(G_{K}\right)$, on note (pour simplifier) $\operatorname{Ind}_{L / K} M$ le $\mathbb{Z}_{p}\left[G_{K}\right]$-module $\operatorname{Ind}_{L / K}\left(\operatorname{Res}_{L / K} M\right)$. Le module $\operatorname{Ind}_{L / K} M$ a alors une structure naturelle de $\mathbb{Z}_{p}[G]$-module donnée par la formule $\bar{g}(\sigma \otimes m)=\sigma g^{-1} \otimes g(m)$, où $\bar{g}$ désigne l'image de $g \in G_{K}$ dans $G$, et on a des isomorphismes canoniques :

$$
\begin{aligned}
& M^{G_{L}} \simeq\left(\operatorname{Ind}_{L / K} M\right)^{G_{K}}, \quad \quad m \mapsto \sum_{\bar{g} \in G} g \otimes m ; \\
& \operatorname{Ind}_{L / K}(M) \simeq\left(\mathbb{Z}_{p}[G] \otimes_{\mathbb{Z}_{p}} M\right)^{\iota}, \quad \sigma \otimes m \mapsto \bar{\sigma} \otimes \sigma(m) .
\end{aligned}
$$

Soit $\boldsymbol{M}\left(G_{K}\right)^{\text {ind }}$ la sous-catégorie de $\boldsymbol{M}\left(G_{K}\right)$ dont les objets sont les limites inductives de $\mathbb{Z}_{p}\left[G_{K}\right]$-modules de type fini sur $\mathbb{Z}_{p}$. Pour tout $M \in \boldsymbol{M}\left(G_{K}\right)^{\text {ind }}$, on note $C^{\bullet}\left(G_{K}, \operatorname{Ind}_{L / K} M\right)$ le complexe des cochaînes continues de $G_{K}$ à valeurs dans $\operatorname{Ind}_{L / K}(M)$. On obtient ainsi un foncteur de $\boldsymbol{M}\left(G_{K}\right)^{\text {ind }}$ dans $\boldsymbol{D}\left(\mathbb{Z}_{p}[G]\right)$ qui à $M$ associe $C^{\bullet}\left(G_{K}, \operatorname{Ind}_{L / K} M\right)$ et qui induit un foncteur exact :

$$
\mathbb{R} \Gamma(L, \cdot): \boldsymbol{D}\left(\boldsymbol{M}\left(G_{K}\right)^{\text {ind }}\right) \rightarrow \boldsymbol{D}\left(\mathbb{Z}_{p}[G]\right) .
$$

Le lemme de Shapiro donne un isomorphisme $\mathbb{R}^{i} \Gamma(L, M) \simeq H^{i}(L, M)$.

Proposition 2.7. Si $L / K$ est une extension abélienne finie et si $M$ est un $\mathbb{Z}_{p}\left[G_{K}\right]$ module qui est de type fini sur $\mathbb{Z}_{p}$, alors :

(1) $\mathbb{R} \Gamma(L, M) \in \boldsymbol{D}^{p}\left(\mathbb{Z}_{p}[G]\right)$;

(2) si de plus $M$ est de $\mathbb{Z}_{p}$-torsion, alors $\operatorname{det}_{\mathbb{Z}_{p}[G]} \mathbb{R} \Gamma(L, M)=\operatorname{det}_{\mathbb{Z}_{p}[G]}^{-1}\left(\operatorname{Ind}_{L / \mathbb{Q}_{p}} M\right)$ dans $Q\left(\mathbb{Z}_{p}[G]\right)$.

Preuve. Voir [Kat93c] et [BF96].

Pour terminer ce paragraphe, faisons le lien entre les constructions ci-dessus et la théorie d'Iwasawa des représentations $p$-adiques. Rappelons que l'on a fixé un système compatible $\left(\zeta_{p^{n}}\right)_{n \geqslant 0}$ de racines primitives $p^{n}$-ièmes de l'unité et posé $K_{n}=$ $K\left(\zeta_{p^{n}}\right)$ et $K_{\infty}=\bigcup_{n \geqslant 1} K_{n}$. Soient $G_{n}=\operatorname{Gal}\left(K_{n} / K\right), H_{K}=\operatorname{Gal}\left(\overline{\mathbb{Q}}_{p} / K_{\infty}\right), \Gamma=$ $\operatorname{Gal}\left(K_{\infty} / K\right), \Gamma_{n}=\operatorname{Gal}\left(K_{\infty} / K_{n}\right)$ et enfin $\Lambda=\mathbb{Z}_{p}[[\Gamma]]$ l'algèbre d'Iwasawa de $\Gamma$.

Si $T$ est une $\mathbb{Z}_{p}$-représentation de $G_{K}$, alors le module induit $\operatorname{Ind}_{K_{\infty} / K}(T)$ est isomorphe à $\left(\Lambda \otimes_{\mathbb{Z}_{p}} T\right)^{\iota}$ et on pose $\mathbb{R} \Gamma_{\mathrm{Iw}}(K, T)=\mathbb{R} \Gamma\left(K, \operatorname{Ind}_{K_{\infty} / K}(T)\right)$. La proposition suivante est un cas particulier d'un résultat de Nekovář (voir [Nek02, proposition 8.4.22]). 
Proposition 2.8. (1) On a des isomorphismes canoniques :

$$
\mathbb{R}^{i} \Gamma_{\mathrm{IW}}(K, T) 0 \simeq H_{\mathrm{IW}}^{i}(K, T) .
$$

(2) Dans la catégorie $\boldsymbol{D}\left(\mathbb{Z}_{p}\left[G_{n}\right]\right)$, on a un isomorphisme canonique :

$$
\mathbb{Z}_{p}\left[G_{n}\right] \otimes_{\Lambda}^{L} \mathbb{R} \Gamma_{\mathrm{Iw}}(K, T) \simeq \mathbb{R} \Gamma\left(K_{n}, T\right) .
$$

(3) On a une suite spectrale dégénérée :

$$
E_{2}^{i j}=H^{i}\left(K_{\infty} / K_{n}, H_{\mathrm{IW}}^{j}(K, T)\right) \Rightarrow H^{i+j-1}\left(K_{n}, T\right),
$$

qui donne lieu à des suites exactes :

$$
0 \rightarrow H_{\mathrm{Iw}}^{j}(K, T)_{\Gamma_{n}} \rightarrow H^{j}\left(K_{n}, T\right) \rightarrow H_{\mathrm{Iw}}^{j+1}(K, T)^{\Gamma_{n}} \rightarrow 0 .
$$

Remarque 2.9. La proposition 2.8 donne une approche unifiée des isomorphismes et suites exactes bien connus en théorie d'Iwasawa locale.

(1) On a $H_{\mathrm{Iw}}^{0}(K, T)=0$ ce qui fournit un isomorphisme :

$$
H^{0}\left(K_{n}, T\right)=H_{\mathrm{IW}}^{1}(K, T)^{\Gamma_{n}} .
$$

(2) Par la dualité locale, on a un isomorphisme

$$
H_{\mathrm{Iw}}^{2}(K, T) \simeq H^{0}\left(K_{\infty}, V^{*}(1) / T^{*}(1)\right)^{\wedge}
$$

où $\wedge$ signifie le dual de Pontryagin. En particulier, $H_{\mathrm{Iw}}^{2}(K, T)$ est un $\Lambda$-module de type fini de torsion. Pour $j=2$, la suite exacte (3) de la proposition 2.8 donne un isomorphisme :

$$
H_{\mathrm{IW}}^{2}(K, T)_{\Gamma_{n}} \simeq H^{2}\left(K_{n}, T\right)
$$

qui est le dual de l'isomorphisme évident :

$$
H^{0}\left(K_{n}, V^{*}(1) / T^{*}(1)\right) \simeq H^{0}\left(K_{\infty}, V^{*}(1) / T^{*}(1)\right)^{\Gamma_{n}} .
$$

(3) Comme le groupe $\Gamma$ est de dimension cohomologique 1, on a une suite exacte « inflation - restriction » :

$$
\begin{aligned}
0 \rightarrow & H^{1}\left(\Gamma_{n},\left(V^{*}(1) / T^{*}(1)\right)^{H_{K}}\right) \rightarrow H^{1}\left(K_{n}, V^{*}(1) / T^{*}(1)\right) \\
& \rightarrow H^{1}\left(K_{\infty}, V^{*}(1) / T^{*}(1)\right)^{\Gamma_{n}} \rightarrow 0
\end{aligned}
$$

En dualisant cette suite exacte, on obtient une suite exacte (voir la proposition 3.2.1 de [Per94]) :

$$
\begin{aligned}
0 \rightarrow & H_{\mathrm{IW}}^{1}(K, T)_{\Gamma_{n}} \rightarrow H^{1}\left(K_{n}, T\right) \\
& \rightarrow H^{1}\left(\Gamma_{n},\left(V^{*}(1) / T^{*}(1)\right)^{H_{K}}\right)^{\wedge} \rightarrow 0 .
\end{aligned}
$$


Comme :

$$
\begin{aligned}
H^{1}\left(\Gamma_{n}, H^{0}\left(K_{\infty}, V^{*}(1) / T^{*}(1)\right)\right)^{\wedge} & \simeq H^{0}\left(\Gamma_{n}, H^{0}\left(K_{\infty}, V^{*}(1) / T^{*}(1)\right)^{\wedge}\right) \\
& \simeq H_{\mathrm{Iw}}^{2}(K, T)^{\Gamma_{n}},
\end{aligned}
$$

on obtient une suite exacte :

$$
0 \rightarrow H_{\mathrm{IW}}^{1}(K, T)_{\Gamma_{n}} \rightarrow H^{1}\left(K_{n}, T\right) \rightarrow H_{\mathrm{IW}}^{2}(K, T)^{\Gamma_{n}} \rightarrow 0,
$$

qui coïncide avec la suite (3) de la proposition 2.8 pour $j=1$.

(4) $\mathrm{Si}\left(V^{*}\right)^{H_{K}}=0$, alors $H_{\mathrm{Iw}}^{2}(K, T)$ est fini et on a :

$$
\sharp H_{\mathrm{IW}}^{2}(K, T)^{\Gamma_{n}}=\sharp H_{\mathrm{IW}}^{2}(K, T)_{\Gamma_{n}}=\sharp H^{0}\left(K_{n}, V^{*}(1) / T^{*}(1)\right) .
$$

(5) Dans [Per92, §2], Perrin-Riou montre que $H_{\mathrm{Iw}}^{1}(K, T)$ est un $\Lambda$-module de rang $\left[K: \mathbb{Q}_{p}\right] \operatorname{dim}(V)$. L'application d'inflation :

$$
\left(T^{H_{K}}\right)_{\Gamma_{n}} \simeq H^{1}\left(\Gamma_{n}, T^{H_{K}}\right) \hookrightarrow H^{1}\left(K_{n}, T\right)
$$

induit une injection :

$$
T^{H_{K}} \simeq \lim _{\leftarrow} H^{1}\left(\Gamma_{n}, T^{H_{K}}\right) \hookrightarrow H_{\mathrm{Iw}}^{1}(K, T),
$$

qui identifie $T^{H_{K}}$ avec la $\Lambda$-torsion de $H_{\mathrm{Iw}}^{1}(K, T)$.

2.3. Constantes locales des représentations de Weil-Deligne. L'objet de ce paragraphe est de fournir des rappels sur la théorie des constantes locales, telle qu'elle est développée dans [Del73], auquel nous renvoyons pour plus de détails. Le corps $K$ est toujours une extension finie de $\mathbb{Q}_{p}$. On fixe une uniformisante $\pi_{K}$ de $K$ et on note $|\cdot|_{K}$ la norme de $K$ normalisée par $\left|\pi_{K}\right|_{K}=q_{K}^{-1}$ où $q_{K}$ est le cardinal du corps résiduel $k_{K}$ de $K$.

On note $K^{\mathrm{nr}}$ l'extension maximale non-ramifiée de $K$ et $\mathrm{Fr}_{K}$ le Frobenius géométrique de $K^{\mathrm{nr}}$. Le groupe de Weil $W_{K}$ de $K$ est par définition le sous-groupe de $G_{K}$ formé des $g \in G_{K}$ tels que la restriction de $g$ à $K^{\mathrm{nr}}$ soit une puissance entière de $\mathrm{Fr}_{K}$. On a donc une suite exacte :

$$
0 \rightarrow I_{K} \rightarrow W_{K} \stackrel{v}{\rightarrow} \mathbb{Z} \rightarrow 0,
$$

où l'application $v$ est définie par la formule $w_{\mid K^{\mathrm{nr}}}=\operatorname{Fr}_{K}^{v(w)}$.

Soit $E$ un corps de caractéristique 0 et contenant toutes les racines de l'unité d'ordre une puissance de $p$ et d'ordre $p-1$. On fixe une mesure de Haar $\mu_{K}$ sur $K$ et un caractère additif continu $\psi: K \rightarrow E^{\times}$(le corps $E$ étant muni de la topologie 
discrète). Comme $\psi$ est continu, il est trivial sur un sous-groupe ouvert de $K$ et l'on définit son conducteur $n(\psi)$ comme étant le plus grand entier $n$ tel que $\psi$ est trivial $\operatorname{sur} \pi_{K}^{-n} \mathcal{O}_{K}$.

La théorie de Langlands et Deligne (voir [Del73]) associe à toute représentation $E$-linéaire $V$ de $W_{K}$ une constante $\varepsilon\left(V, \psi, \mu_{K}\right)$ vérifiant les propriétés suivantes :

(1) Si $V$ est de dimension 1 , alors $\varepsilon\left(V, \psi, \mu_{K}\right)$ coïncide avec la constante locale «abélienne» définie par la théorie de Tate (dans [Tat67]). Plus précisement, l'isomorphisme de réciprocité $\theta_{K}: K^{\times} \rightarrow W_{K}^{\text {ab }}$ permet de voir $V$ comme un quasi-caractère $\eta: K^{\times} \rightarrow E^{\times}$. On note $a(\eta)$ le conducteur de $\eta$ et on fixe $c \in \mathcal{O}_{K}$ vérifiant $v_{K}(c)=a(\eta)+n(\psi)$. Si $\eta$ est non-ramifié, alors on a :

$$
\varepsilon\left(\eta, \psi, \mu_{K}\right)=\frac{\eta(c)}{|c|_{K}} \int_{\mathcal{O}_{K}} d \mu_{K}
$$

et si $\eta$ est ramifié, alors on a :

$$
\varepsilon\left(\eta, \psi, \mu_{K}\right)=\sum_{n \in \mathbb{Z}} \int_{\left\{v_{K}(x)=n\right\}} \eta^{-1}(x) \psi(x) d \mu_{K}=\int_{c^{-1} \mathcal{O}_{K}} \eta^{-1}(x) \psi(x) d \mu_{K} .
$$

(2) Pour toute suite exacte de représentations $0 \rightarrow V^{\prime} \rightarrow V \rightarrow V^{\prime \prime} \rightarrow 0$, on a $\varepsilon\left(V, \psi, \mu_{K}\right)=\varepsilon\left(V^{\prime}, \psi, \mu_{K}\right) \varepsilon\left(V^{\prime \prime}, \psi, \mu_{K}\right)$.

(3) Pour tout $a \in K^{\times}$, on a $\varepsilon\left(V, \psi, a \mu_{K}\right)=a^{\operatorname{dim} V} \varepsilon\left(V, \psi, \mu_{K}\right)$ et si $m_{a}$ dénote la fonction $x \mapsto a x$, alors $\varepsilon\left(V, \psi \circ m_{a}, \mu_{K}\right)=\operatorname{det}(V)(a)|a|_{K}^{-\operatorname{dim} V} \varepsilon\left(V, \psi, \mu_{K}\right)$.

(4) Si $L$ est une extension finie de $K$, alors on a une constante $\lambda\left(L / K, \psi, \mu_{L}, \mu_{K}\right) \in$ $E$ telle que pour toute représentation $V$ de $W_{L}$ on ait :

$$
\varepsilon\left(\operatorname{Ind}_{L / K}(V), \psi, \mu_{K}\right)=\lambda\left(L / K, \psi, \mu_{L}, \mu_{K}\right)^{\operatorname{dim} V}{ }_{\varepsilon}\left(V, \psi \circ \operatorname{Tr}_{L / K}, \mu_{L}\right) .
$$

(5) Soient $\omega_{1}: K^{\times} \rightarrow E^{\times}$le quasi-caractère donné par la formule $\omega_{1}(a)=|a|_{K}$ et $\mu_{K}^{*}$ la mesure duale de $\mu_{K}$ relativement à $\psi$. On a alors :

$$
\varepsilon\left(V, \psi, \mu_{K}\right) \varepsilon\left(V^{*} \otimes \omega_{1}, \psi \circ m_{-1}, \mu_{K}^{*}\right)=1 .
$$

(6) Pour une représentation non-ramifiée $W$, on a :

$$
\varepsilon\left(V \otimes W, \psi, \mu_{K}\right)=\operatorname{det}(W)\left(\pi_{K}^{a(V)+\operatorname{dim}(V) n(\psi)}\right) \varepsilon\left(V, \psi, \mu_{K}\right)^{\operatorname{dim} W},
$$

où $a(V)$ est le conducteur d'Artin de $V$.

Rappelons que l'on a fixé un système compatible $\left(\zeta_{p^{n}}\right)_{n \geqslant 0}$ de racines de l'unité. On note $\psi_{0}$ l'unique caractère additif de $\mathbb{Q}_{p}$ vérifiant $\psi_{0}\left(1 / p^{n}\right)=\zeta_{p^{n}}$ et on pose $\psi_{K}=\psi_{0} \circ \operatorname{Tr}_{K} / \mathbb{Q}_{p}$. On normalise la mesure $\mu_{K}$ en imposant $\mu_{K}\left(\mathcal{O}_{K}\right)=1$. Soit enfin $(\cdot, \cdot)_{K}: K^{\times} \times K^{\times} \rightarrow\{ \pm 1\}$ le symbole de Hilbert. Le lemme suivant est bien connu des experts. 
Lemme 2.10. Si $L / K$ est une extension finie, alors :

$$
\lambda\left(L / K, \psi_{K}, \mu_{L}, \mu_{K}\right)= \pm\left(-1, d_{L / K}\right)_{K}^{1 / 2}\left|d_{L / K}\right|_{p}^{\left[K: \mathbb{Q}_{p}\right] / 2},
$$

où $d_{L / K}$ est le discriminant de $L / K$.

Preuve. Montrons d'abord que pour tout $a \in K^{\times}$on a :

$$
\operatorname{det}\left(\operatorname{Ind}_{L / K}[1]\right)(a)=\left(a, d_{L / K}\right)_{K} .
$$

Comme $\left(a, d_{L / K}\right)_{K}=\left(\sqrt{d_{L / K}}\right)^{\theta_{K}(a)} / \sqrt{d_{L / K}}$, il suffit de montrer que l'application de Kummer $K^{\times} \rightarrow H^{1}(K,\{ \pm 1\})$ envoie $d_{L / K} \operatorname{sur} \operatorname{det}\left(\operatorname{Ind}_{L / K}[1]\right)$. Soit $G_{K} / G_{L}=$ $\left\{g_{i} G_{L} \mid i=1, \ldots, n\right\}$ une décomposition de $G_{K}$ en classes de $G_{L}$. Le groupe $G_{K}$ agit sur $G_{K} / G_{L}$ ce qui fournit un homomorphisme $\rho: G_{K} \rightarrow S_{n}$. Soit $\varepsilon_{n}: S_{n} \rightarrow\{ \pm 1\}$ la signature. Si $\alpha \in L$ est tel que $L=K(\alpha)$, alors :

$$
\sqrt{d_{L / K}}=\prod_{i<j}\left(g_{i}(\alpha)-g_{j}(\alpha)\right)
$$

et on voit que l'image de $d_{L / K}$ dans $H^{1}(K,\{ \pm 1\})$ coïncide avec $\varepsilon_{n} \circ \rho$. D'autre part, il résulte directement de la définition de $\operatorname{Ind}_{L / K}[1]$ que $\varepsilon_{n} \circ \rho=\operatorname{det}\left(\operatorname{Ind}_{L / K}[1]\right)$, d'où la formule voulue.

Passons maintenant à la démonstration du lemme. Les formules (3) et (5), appliquées à la représentation régulière $\operatorname{Ind}_{L / K}[1]$ donnent :

$$
\varepsilon\left(\operatorname{Ind}_{L / K}[1], \psi_{K}, \mu_{K}\right) \varepsilon\left(\operatorname{Ind}_{L / K}[1] \otimes \omega_{1}, \psi_{K} \circ m_{-1}, \mu_{K}\right)=\left|d_{K}\right|_{p}^{-[L: K]} .
$$

Comme par ailleurs $a\left(\operatorname{Ind}_{L / K}[1]\right)=v_{K}\left(d_{L / K}\right)$ (voir par exemple [Ser68, chapitre IV, proposition 4]) et $n\left(\psi_{K}\right)=v_{K}\left(\mathscr{D}_{K / \mathbb{Q}_{p}}\right)$, on a :

$$
\begin{aligned}
& \varepsilon\left(\operatorname{Ind}_{L / K}[1] \otimes \omega_{1}, \psi_{K} \circ m_{-1}, \mu_{K}\right) \\
& \quad=\left|d_{L}\right|_{p} \operatorname{det}\left(\operatorname{Ind}_{L / K}[1]\right)(-1) \varepsilon\left(\operatorname{Ind}_{L / K}[1], \psi_{K}, \mu_{K}\right) .
\end{aligned}
$$

On a $\left|d_{L}\right|_{p}=\left|d_{L / K}\right|_{p}^{\left[K: \mathbb{Q}_{p}\right]}\left|d_{K}\right|_{p}^{[L: K]}$ et $\operatorname{det}\left(\operatorname{Ind}_{L / K}[1]\right)(-1)=\left(-1, d_{L / K}\right)_{K}$, d'où :

$$
\varepsilon\left(\operatorname{Ind}_{L / K}[1], \psi_{K}, \mu_{K}\right)= \pm\left(-1, d_{L / K}\right)_{K}^{1 / 2}\left|d_{L / K}\right|_{p}^{\left[K: \mathbb{Q}_{p}\right] / 2}\left|d_{L}\right|_{p}^{-1} .
$$

Comme $\varepsilon\left([1], \psi_{L}, \mu_{L}\right)=\left|d_{L}\right|_{p}^{-1}$, on en déduit le lemme.

Remarque 2.11. Il est facile de voir que si $L / K$ est une extension non-ramifiée de degré $f$, alors $\lambda\left(L / K, \psi, \mu_{L}, \mu_{K}\right)=(-1)^{(f-1) n(\psi)}$. 
Supposons maintenant que $K$ est une extension non-ramifiée de $\mathbb{Q}_{p}$ de degré $f$, et notons $X\left(G_{n}\right)$ le groupe des caractères de $G_{n}=\operatorname{Gal}\left(K_{n} / K\right)$ à valeurs dans $E$. Rappelons que pour tout $\eta \in X\left(G_{n}\right)$, on note $e_{\eta}$ l'idempotent habituel. On définit la somme de Gauss $\tau(\eta)$ en posant $\tau(\eta)=\sum_{g \in G_{k}} \eta^{-1}(g) g\left(\zeta_{p^{k}}\right)=\sharp G_{k} e_{\eta}\left(\zeta_{p^{k}}\right)$, où $k=a(\eta)$ est le conducteur de $\eta$.

Lemme 2.12. Pour tout caractère $\eta \in X\left(G_{n}\right)$, on $a$ :

$$
\varepsilon\left(\eta, \psi_{K}, \mu_{K}\right)=(-1)^{(f-1) a(\eta)} \tau(\eta)^{f} .
$$

Preuve. Pour simplifier les notations, on note $\mu_{0}$ la mesure de Haar sur $\mathbb{Q}_{p}$. Comme $K / \mathbb{Q}_{p}$ est non-ramifiée, les groupes de Galois des extensions $\mathbb{Q}_{p}\left(\zeta_{p^{n}}\right) / \mathbb{Q}_{p}$ et $K_{n} / K$ sont isomorphes et $\eta$ peut être vu comme la restriction $\operatorname{Res}_{K} / \mathbb{Q}_{p} \tilde{\eta}$ d'un caractère $\tilde{\eta}: \operatorname{Gal}\left(\mathbb{Q}_{p}\left(\zeta_{p^{n}}\right) / \mathbb{Q}_{p}\right) \rightarrow E^{\times}$. Comme $\lambda\left(K / \mathbb{Q}_{p}, \psi_{0}, \mu_{K}, \mu_{0}\right)=1$, on a :

$$
\begin{aligned}
\varepsilon\left(\eta, \psi_{K}, \mu_{K}\right) & =\varepsilon\left(\operatorname{Ind}_{K / \mathbb{Q}_{p}} \eta, \psi_{0}, \mu_{0}\right) \varepsilon\left(\operatorname{Ind}_{K / \mathbb{Q}_{p}}[1] \otimes \tilde{\eta}, \psi_{0}, \mu_{0}\right) \\
& =(-1)^{(f-1) a(\eta)} \varepsilon\left(\tilde{\eta}, \psi_{0}, \mu_{0}\right)^{f} .
\end{aligned}
$$

Si on suppose que $n=a(\eta)$, alors l'application composée $\mathbb{Q}_{p}^{\times} \rightarrow \operatorname{Gal}\left(\mathbb{Q}_{p}^{\mathrm{ab}} / \mathbb{Q}_{p}\right) \rightarrow$ $G_{n} \simeq\left(\mathbb{Z} / p^{n} \mathbb{Z}\right)^{\times}$envoie $u \in \mathbb{Z}_{p}^{\times}$sur $u \bmod p^{n}$ et $p$ sur 1 , ce qui fait que :

$$
\begin{aligned}
\varepsilon\left(\tilde{\eta}, \psi_{0}, \mu_{0}\right) & =p^{n} \sum_{u \in \mathbb{Z}_{p}^{\times} / 1+p^{n} \mathbb{Z}_{p}} \mu_{0}\left(1+p^{k} \mathbb{Z}_{p}\right) \tilde{\eta}(u)^{-1} \zeta_{p^{n}}^{u} \\
& =\sum_{u \in \mathbb{Z}_{p}^{\times} / 1+p^{n} \mathbb{Z}_{p}} \tilde{\eta}(u)^{-1} \zeta_{p^{n}}^{u}=\tau(\eta) .
\end{aligned}
$$

Le cas général s'en déduit.

On appelle représentation du groupe de Weil-Deligne un couple $(\rho, N)$ formé d'une représentation $\rho: W_{K} \rightarrow \operatorname{Aut}_{E}(V)$ du groupe de Weil $W_{K}$ et d'un endomorphisme nilpotent $N: V \rightarrow V$ vérifiant $\rho(w)^{-1} N \rho(w)=q_{K}^{\nu(w)} N$ (voir [Del73, §8]). On pose alors :

$$
\varepsilon\left(V, \psi_{K}, \mu_{K}\right)=\varepsilon\left(\rho, \psi_{K}, \mu_{K}\right) \operatorname{det}\left(-\operatorname{Fr}_{K} \mid V^{I_{K}} /\left(V^{I_{K}}\right)^{N=0}\right) .
$$

2.4. Constantes locales des représentations potentiellement semi-stables. Pour plus de détails, voir [FP94, chapitre I, §1.3]. On garde les notations et les conventions des paragraphes précédents. En particulier, $K$ est toujours une extension finie de $\mathbb{Q}_{p}$ et $K_{0}$ est son sous-corps maximal non-ramifié, dont le degré sur $\mathbb{Q}_{p}$ est $f=\left[K_{0}: \mathbb{Q}_{p}\right]$. Rappelons que l'on a défini ci-dessus un caractère additif $\psi_{K}$ à valeurs dans $\mathbb{Q}_{p}\left(\zeta_{p^{\infty}}\right)=\bigcup_{n \geqslant 0} \mathbb{Q}_{p}\left(\zeta_{p^{n}}\right)$ en posant $\psi_{K}\left(a / p^{n}\right)=\zeta_{p^{n}}^{\operatorname{Tr}_{K} \mathbb{Q}_{p}(a)}$. On fixe une extension abélienne finie $L / K$ et on pose toujours $G=\operatorname{Gal}(L / K)$.

Le lemme suivant est laissé en exercice au lecteur. 
Lemme 2.13. Si $L / K$ est une extension finie et si $V$ est une représentation p-adique de $G_{L}$, alors $\boldsymbol{D}_{\mathrm{dR}}^{K}\left(\operatorname{Ind}_{L / K} V\right) \simeq \boldsymbol{D}_{\mathrm{dR}}^{L}(V)$ et $\boldsymbol{D}_{\mathrm{pst}}\left(\operatorname{Ind}_{L / K} V\right) \simeq \operatorname{Ind}_{L / K} \boldsymbol{D}_{\mathrm{pst}}(V)$.

Si $V$ est une représentation potentiellement semi-stable de $G_{K}$, alors la représentation $\operatorname{Ind}_{L / K} V \simeq\left(\mathbb{Q}_{p}[G] \otimes_{\mathbb{Q}_{p}} V\right)^{\iota}$ est bien-sûr elle aussi potentiellement semistable et $D=\boldsymbol{D}_{\mathrm{pst}}\left(\operatorname{Ind}_{L / K} V\right)$ est un $K_{0}^{\mathrm{nr}}[G]$-module muni d'une action naturelle $\mathrm{Fr}_{K}$-semi-linéaire de $W_{K}$. On munit $D$ d'une action linéaire de $W_{K}, \rho: W_{K} \rightarrow$ $\operatorname{Aut}_{K_{0}^{\mathrm{nr}}[G]}(D)$ en posant $(\rho(w))(d)=w \varphi^{f v(w)}(d)$ où l'application $v$ est celle définie au paragraphe 2.3. Le module $D$ est muni d'un opérateur de monodromie $N$ vérifiant $N \circ \varphi=p \varphi \circ N$ ce qui fait que $\rho(w)^{-1} N \rho(w)=q_{K}^{\nu(w)}$ et que $(\rho, N)$ est une représentation du groupe de Weil-Deligne. On pose alors :

$$
\varepsilon(L / K, V)=\varepsilon\left(D, \psi_{K}, \mu_{K}\right)=\varepsilon\left(\rho, \psi_{K}, \mu_{K}\right) \operatorname{det}\left(-\operatorname{Fr}_{K} \mid D^{I_{K}} /\left(D^{I_{K}}\right)^{N=0}\right) .
$$

Il est facile de voir (cf. [FP94, remarque 1.3.3]) que la représentation $\rho$ est $\mathbb{Q}_{p}$-rationnelle, d'où l'on tire que $\varepsilon(L / K, V) \in \mathbb{Q}_{p}\left(\zeta_{p^{\infty}}\right)[G]$.

Si $E$ est un corps contenant $K^{\mathrm{nr}}$ ainsi que les valeurs des caractères de $G$, alors on a :

$$
E[G]=\bigoplus_{\eta \in X(G)} E_{\eta}, \quad \text { où } \quad E_{\eta}=e_{\eta} E,
$$

et le module $D$ se décompose sur $E$ en produit de ses $\eta$-composantes : $D_{E}=$ $\bigoplus_{\eta \in X(G)} D_{\eta}$. On appelle $\eta_{0}$ le caractère trivial. On déduit de la décomposition cidessus que $\varepsilon(L / K, V)=\sum_{\eta \in X(G)} \varepsilon\left(D_{\eta}, \psi_{K, \eta}, \mu_{K}\right)$, avec $\varepsilon\left(D_{\eta}, \psi_{K, \eta}, \mu_{K}\right) \in E_{\eta}$.

Si $V$ est une représentation potentiellement semi-stable de $G_{K}$, alors par le lemme 2.13 ci-dessus, $\boldsymbol{D}_{\mathrm{dR}}^{\mathbb{Q}_{p}}\left(\operatorname{Ind}_{L / \mathbb{Q}_{p}}(V)\right) \simeq \boldsymbol{D}_{\mathrm{dR}}^{L}(V)$ et on a donc un isomorphisme canonique :

$$
\operatorname{comp}_{V, L / \mathbb{Q}_{p}}: \boldsymbol{B}_{\mathrm{dR}} \otimes_{\mathbb{Q}_{p}} \operatorname{Ind}_{L / \mathbb{Q}_{p}} V \simeq \boldsymbol{B}_{\mathrm{dR}} \otimes_{\mathbb{Q}_{p}} \boldsymbol{D}_{\mathrm{dR}}^{L}(V) .
$$

On en déduit un homomorphisme :

$$
\tilde{\alpha}_{V, L / K}: \operatorname{det}_{\mathbb{Q}_{p}[G]}^{-1}\left(D_{\mathrm{dR}}^{L}(V)\right) \otimes \operatorname{det}_{\mathbb{Q}_{p}[G]}\left(\operatorname{Ind}_{L / \mathbb{Q}_{p}} V\right) \rightarrow \mathbb{Q}_{p}[G] \otimes_{\mathbb{Q}_{p}} \boldsymbol{B}_{\mathrm{dR}}
$$

On voit que $\operatorname{det}_{\mathbb{Q}_{p}[G]}\left(\operatorname{Ind}_{L / \mathbb{Q}_{p}} V\right)$ est une $\mathbb{Q}_{p}[G]$-représentation de de Rham de rang 1 et de poids $r=-\left[K: \mathbb{Q}_{p}\right] t_{H}(V)$ et il existe donc une extension abélienne

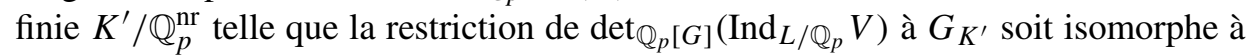
$\mathbb{Q}_{p}[G](r)$. On en déduit donc une application

$$
\alpha_{V, L / K}: \operatorname{det}_{\mathbb{Q}_{p}[G]}^{-1}\left(\boldsymbol{D}_{\mathrm{dR}}^{L}(V)\right) \otimes \operatorname{det}_{\mathbb{Q}_{p}[G]}\left(\operatorname{Ind}_{L / \mathbb{Q}_{p}} V\right) \rightarrow K^{\prime}[G],
$$

donnée par la formule $\alpha_{V, L / K}=t^{-r} \tilde{\alpha}_{V, L / K}$, où $t=\log [\varepsilon] \in \boldsymbol{B}_{\mathrm{dR}}$ est l'uniformisante de $\boldsymbol{B}_{\mathrm{dR}}^{+}$associée à $\varepsilon=\left(\zeta_{p^{n}}\right)_{n \geqslant 0}$.

Soit $\hat{\sigma}$ l'élément de $\operatorname{Gal}\left(\mathbb{Q}_{p}^{\mathrm{ab}} / \mathbb{Q}_{p}\right)$ qui opère trivialement sur les racines $p^{n}$-ièmes de l'unité et dont la restriction à $\mathbb{Q}_{p}^{\text {nr }}$ est égale à $\sigma$, et soit

$$
a_{V, L / K}=\operatorname{det}_{\mathbb{Q}_{p}[G]}\left(\operatorname{Ind}_{L / \mathbb{Q}_{p}} V\right)(\hat{\sigma}) \in \mathbb{Z}_{p}[G]^{\times} .
$$


Définition 2.14. On pose : $\mathbb{Z}_{p}[G]_{V, L / K}=\left\{x \in \widehat{\mathbb{Z}_{p}^{\mathrm{nr}}}[G] \mid \sigma(x)=a_{V, L / K} x\right\}$ et $\mathbb{Q}_{p}[G]_{V, L / K}=\mathbb{Q}_{p} \otimes_{\mathbb{Z}_{p}} \mathbb{Z}_{p}[G]_{V, L / K}$

Le module $\mathbb{Z}_{p}[G]_{V, L / K}$ est alors libre de rang 1 sur $\mathbb{Z}_{p}[G]$ (voir [Kat93b]). Posons

$$
\Gamma^{*}(i)= \begin{cases}(i-1) ! & \text { si } i>0, \\ \frac{(-1)^{i}}{(-i) !} & \text { si } i \leqslant 0,\end{cases}
$$

et $\Gamma^{*}(V)=\prod_{i \in \mathbb{Z}} \Gamma^{*}(-i)^{h_{i}(V)\left[K: \mathbb{Q}_{p}\right]}$. Soit aussi :

$$
\beta_{V, L / K}=\lambda\left(K / \mathbb{Q}_{p}\right)^{-\operatorname{dim} V} \Gamma^{*}(V) \varepsilon(L / K, V)^{-1} \alpha_{V, L / K},
$$

où $\lambda\left(K / \mathbb{Q}_{p}\right)=\lambda\left(K / \mathbb{Q}_{p}, \psi_{0}, \mu_{K}, \mu_{0}\right)$ est la constante définie dans le paragraphe 2.3.

Lemme 2.15. L'application $\beta_{V, L / K}$ induit un isomorphisme :

$$
\beta_{V, L / K}: \operatorname{det}_{\mathbb{Q}_{p}[G]}^{-1}\left(D_{\mathrm{dR}}^{L}(V)\right) \otimes \operatorname{det}_{\mathbb{Q}_{p}[G]}\left(\operatorname{Ind}_{L / \mathbb{Q}_{p}} V\right) \rightarrow \mathbb{Q}_{p}[G]_{V, L / K}
$$

Preuve. On note $\chi: G_{K} \rightarrow \mathbb{Z}_{p}^{\times}$le caractère cyclotomique. Si on pose

$$
D=\boldsymbol{D}_{\mathrm{pst}}\left(\operatorname{Ind}_{L / K}(V)\right)=\left(\mathbb{Q}_{p}[G] \otimes_{\mathbb{Q}_{p}} \boldsymbol{D}_{\mathrm{pst}}(V)\right)^{\iota},
$$

alors on a (voir le paragraphe 2.3) :

$$
\frac{\lambda\left(K / \mathbb{Q}_{p}\right)^{\operatorname{dim} V_{\varepsilon}\left(D, \psi_{K}, \mu_{K}\right)}}{\varepsilon\left(\operatorname{Ind}_{K / \mathbb{Q}_{p}} D, \psi_{0}, \mu_{0}\right)} \in \mathbb{Q}_{p}[G] .
$$

Pour tout $g \in G_{\mathbb{Q}_{p}}$, on a :

$$
\begin{aligned}
g\left(\varepsilon\left(\operatorname{Ind}_{K / \mathbb{Q}_{p}} D, \psi_{0}, \mu_{0}\right)\right) & =\varepsilon\left(\operatorname{Ind}_{K / \mathbb{Q}_{p}} D, \psi_{0} \circ m_{\chi}(g), \mu_{0}\right) \\
& =\operatorname{det}_{\mathbb{Q}_{p}[G]}\left(\operatorname{Ind}_{L / \mathbb{Q}_{p}} \boldsymbol{D}_{\mathrm{pst}}(V)\right)(\chi(g)) \varepsilon\left(\operatorname{Ind}_{K / \mathbb{Q}_{p}} D, \psi_{0}, \mu_{0}\right) .
\end{aligned}
$$

D'autre part, si $x \in \operatorname{det}_{\mathbb{Q}_{p}[G]}^{-1}\left(D_{\mathrm{dR}}^{L}(V)\right) \otimes \operatorname{det}_{\mathbb{Q}_{p}[G]}\left(\operatorname{Ind}_{L / \mathbb{Q}_{p}} V\right)$, alors :

$$
\begin{aligned}
g\left(\alpha_{V, L / K}(x)\right) & =\chi^{-r}(g) \operatorname{det}_{\mathbb{Q}_{p}[G]}\left(\operatorname{Ind}_{L / \mathbb{Q}_{p}} V\right)(g) \alpha_{V, L / K}(x) \\
& =\operatorname{det}_{\mathbb{Q}_{p}[G]}\left(\operatorname{Ind}_{L / \mathbb{Q}_{p}} \boldsymbol{D}_{\mathrm{pst}}(V)\right)(g) \alpha_{V, L / K}(x) .
\end{aligned}
$$

On en déduit le lemme.

On donne maintenant une formule explicite pour l'application $\beta_{V, K_{n} / K}$ pour les représentations absolument cristallines, formule qui est utilisée dans la suite. On suppose donc que $K$ est non-ramifiée, et on écrit comme ci-dessus $f=\left[K: \mathbb{Q}_{p}\right]$, $q_{K}=p^{f}$ et $d=\operatorname{dim} V$. 
Lemme 2.16. Si $V$ est une représentation cristalline de $G_{K}$, alors :

$$
\varepsilon\left(D_{\eta}, \psi_{K, \eta}, \mu_{K}\right)=\operatorname{det}\left(\varphi \mid \boldsymbol{D}_{\text {cris }}(V)\right)^{a(\eta)} \tau\left(\eta^{-1}\right)^{f d} \otimes e_{\eta}^{\iota} .
$$

Preuve. Comme $D=\boldsymbol{D}_{\mathrm{pst}}\left(\operatorname{Ind}_{K_{n} / K} V\right)=\left(K^{\mathrm{nr}}\left[G_{n}\right] \otimes_{K} \boldsymbol{D}_{\text {cris }}(V)\right)^{\iota}$, on a $D_{\eta}=$ $e_{\eta}^{\iota} \mathbb{Q}_{p}^{\text {ab }} \otimes_{K} \boldsymbol{D}_{\text {cris }}(V)$. L'action naturelle du groupe de Weil sur $\boldsymbol{D}_{\text {cris }}(V)$ est triviale ce qui fait que l'action linéarisée $\rho$ est non-ramifiée et est donnée par la formule $\rho\left(\operatorname{Fr}_{K}\right)=\varphi^{f}$. D'autre part, $G_{K}$ opère sur $e_{\eta}^{\iota}$ par le caractère $\eta^{-1}$. Comme $K / \mathbb{Q}_{p}$ est non-ramifiée, on a $n\left(\psi_{K}\right)=0$ et la formule (6) du paragraphe 2.3 appliquée à $V=e_{\eta}^{\imath} \mathbb{Q}_{p}^{\mathrm{ab}}$ et $W=\boldsymbol{D}_{\text {cris }}(V)$ nous donne :

$$
\varepsilon\left(D_{\eta}, \psi_{\eta}, \mu_{K}\right)=\varepsilon\left(\eta^{-1}, \psi_{K}, \mu_{K}\right)^{d} \operatorname{det}_{K}\left(\varphi^{f} \mid \boldsymbol{D}_{\text {cris }}(V)\right)^{a(\eta)} \otimes e_{\eta}^{\ell} .
$$

Par le lemme 2.5, on $\operatorname{adet}_{K}\left(\varphi^{f} \mid \boldsymbol{D}_{\text {cris }}(V)\right)=(-1)^{(f-1) d} \operatorname{det}\left(\varphi \mid \boldsymbol{D}_{\text {cris }}(V)\right)$ et par le lemme 2.12 , on a $\varepsilon\left(\eta^{-1}, \psi_{K}, \mu_{K}\right)=(-1)^{(f-1) a(\eta)} \tau\left(\eta^{-1}\right)^{f}$. On en déduit le lemme.

Soit $x_{n}=\zeta_{p}+\zeta_{p^{2}}+\cdots+\zeta_{p^{n}}$ et soit $R_{n}$ le $\mathcal{O}_{K}\left[G_{n}\right]$-réseau de $K_{n}$ engendré par $x_{n}$. On fixe un $\mathcal{O}_{K}$-réseau $M$ de $\boldsymbol{D}_{\text {cris }}(V)$ et $T$ un réseau de $V$ et on pose $M_{n}=R_{n} \otimes_{\mathcal{O}_{K}} M$. La restriction de $\operatorname{Ind}_{K / \mathbb{Q}_{p}}(V)$ à $G_{K}$ est manifestement cristalline ce qui implique que la restriction $\operatorname{de} \operatorname{det}_{\mathbb{Q}_{p}}\left(\operatorname{Ind}_{K / \mathbb{Q}_{p}}(V)\right)$ à $G_{K}$ est isomorphe à $\mathbb{Q}_{p}(r)$. Pour $L=K$, l'application $\alpha_{V, L / K}$ s'écrit donc :

$$
\alpha_{V, K}: \operatorname{det}_{\mathbb{Q}_{p}}^{-1} \boldsymbol{D}_{\mathrm{dR}}^{K}(V) \otimes \operatorname{det}_{\mathbb{Q}_{p}}\left(\operatorname{Ind}_{K / \mathbb{Q}_{p}}(V)\right) \rightarrow \mathbb{Q}_{p}^{\mathrm{nr}} .
$$

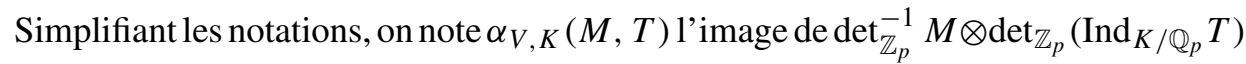
et on pose :

$$
\beta_{V, K_{n} / K}(M, T)=\beta_{V, K_{n} / K}\left(\operatorname{det}_{\mathbb{Z}_{p}\left[G_{n}\right]}^{-1}\left(M_{n}\right) \otimes \operatorname{det}_{\mathbb{Z}_{p}\left[G_{n}\right]}\left(\operatorname{Ind}_{K_{n} / K} T\right)\right) .
$$

Proposition 2.17. Si $V$ est une représentation cristalline de $G_{K}$ et $T$ un réseau de $V$, alors :

$$
\begin{aligned}
& \beta_{V, K_{n} / K}(M, T) \\
& \quad=\Gamma^{*}(V) q_{K}^{-n d}\left(\sum_{\eta \neq 1} \operatorname{det}\left(\varphi \mid \boldsymbol{D}_{\text {cris }}(V)\right)^{-a(\eta)} e_{\eta}+(-1)^{f d} q_{K}^{d} e_{1}\right) \alpha_{V, K}(M, T) .
\end{aligned}
$$

Preuve. Si $G$ est un groupe abélien fini et $M$ un $G$-module, alors on a un isomorphisme canonique $M \simeq\left(M \otimes \mathbb{Z}[G]^{\iota}\right)^{G}$ qui envoie $m \in M$ sur $\sum_{g \in G} g(m) \otimes g^{-1}$. En particulier, si $M$ est un $\mathbb{Q}_{p}[G]$-module et $E$ une extension finie de $\mathbb{Q}_{p}$ contenant les valeurs des caractères de $G$, alors après extension des scalaires on obtient un isomorphisme $M_{E} \simeq\left(M_{E} \otimes_{E} E[G]^{\iota}\right)^{G}$ qui envoie $e_{\eta}(m)$ sur $e_{\eta}(m) \otimes \sharp G e_{\eta}^{\iota}$ pour tous les $m \in M, \eta \in X(G)$. 
Si $V$ est une représentation cristalline, alors l'isomorphisme entre $\boldsymbol{D}_{\mathrm{dR}}^{K_{n}}(V)$ et $\boldsymbol{D}_{\mathrm{dR}}^{\mathbb{Q}_{p}}\left(\operatorname{Ind}_{K_{n} / \mathbb{Q}_{p}}(V)\right)$ peut être explicité comme suit. Le plongement canonique $\boldsymbol{B}_{\text {cris }} \otimes_{\mathbb{Q}_{p}} \mathbb{Q}_{p}\left(\zeta_{p^{n}}\right) \hookrightarrow \boldsymbol{B}_{\mathrm{dR}}$ induit un homomorphisme naturel :

$$
\begin{gathered}
\left(\operatorname{Ind}_{K / \mathbb{Q}_{p}}(V) \otimes_{\mathbb{Q}_{p}} \boldsymbol{B}_{\text {cris }}\right) \otimes_{\mathbb{Q}_{p}}\left(\mathbb{Q}_{p}\left(\zeta_{p^{n}}\right) \otimes_{\mathbb{Q}_{p}} \mathbb{Q}_{p}\left[G_{n}\right]^{l}\right) \\
\rightarrow\left(\left(\operatorname{Ind}_{K / \mathbb{Q}_{p}}(V) \otimes_{\mathbb{Q}_{p}} \mathbb{Q}_{p}\left[G_{n}\right]^{l}\right) \otimes_{\mathbb{Q}_{p}} \boldsymbol{B}_{\mathrm{dR}} .\right.
\end{gathered}
$$

Le théorème de Hilbert $90\left(H^{1}\left(\operatorname{Gal}\left(K / \mathbb{Q}_{p}\right), \mathrm{GL}_{d}(K)\right)=\{1\}\right)$ et le fait que $K / \mathbb{Q}_{p}$ est non ramifiée nous donnent $\boldsymbol{D}_{\text {cris }}\left(\operatorname{Ind}_{K / \mathbb{Q}_{p}}(V)\right) \simeq \boldsymbol{D}_{\text {cris }}(V)$. Comme $\operatorname{Ind}_{K / \mathbb{Q}_{p}}(V) \otimes_{\mathbb{Q}_{p}}$ $\mathbb{Q}_{p}\left[G_{n}\right]^{l} \simeq \operatorname{Ind}_{K_{n} / \mathbb{Q}_{p}}(V)$ et $\left(\mathbb{Q}_{p}\left(\zeta_{p^{n}}\right) \otimes_{\mathbb{Q}_{p}} \mathbb{Q}_{p}\left[G_{n}\right]^{\iota}\right)^{G_{n}} \simeq \mathbb{Q}_{p}\left(\zeta_{p^{n}}\right)$, on en déduit l'isomorphisme voulu :

$$
\boldsymbol{D}_{\mathrm{dR}}^{K_{n}}(V) \simeq \boldsymbol{D}_{\text {cris }}(V) \otimes_{\mathbb{Q}_{p}} \mathbb{Q}_{p}\left(\zeta_{p^{n}}\right) \simeq \boldsymbol{D}_{\mathrm{dR}}^{\mathbb{Q}_{p}}\left(\operatorname{Ind}_{K_{n} / \mathbb{Q}_{p}}(V)\right) .
$$

Soient comp cris $_{\text {i }}: \boldsymbol{B}_{\text {cris }} \otimes_{\mathbb{Q}_{p}} \operatorname{Ind}_{K / \mathbb{Q}_{p}}(V) \stackrel{\sim}{\rightarrow} \boldsymbol{B}_{\text {cris }} \otimes_{\mathbb{Q}_{p}} \boldsymbol{D}_{\text {cris }}(V)$ et $\operatorname{comp}_{V, K_{n} / \mathbb{Q}_{p}}:$ $\boldsymbol{B}_{\mathrm{dR}} \otimes_{\mathbb{Q}_{p}} \operatorname{Ind}_{K_{n} / K}(V) \stackrel{\sim}{\rightarrow} \boldsymbol{B}_{\mathrm{dR}} \otimes_{\mathbb{Q}_{p}} \boldsymbol{D}_{\mathrm{dR}}^{K_{n}}(V)$ les isomorphismes de comparaison. On a un isomorphisme :

$$
\begin{aligned}
&\left(\mathbb{Q}_{p}\left[G_{n}\right]^{l} \otimes_{\mathbb{Q}_{p}} \mathbb{Q}_{p}\left(\zeta_{p^{n}}\right)\right)^{G_{n}} \otimes_{\mathbb{Q}_{p}} \boldsymbol{B}_{\mathrm{dR}} \stackrel{\sim}{\rightarrow} \mathbb{Q}_{p}\left[G_{n}\right]^{l} \otimes_{\mathbb{Q}_{p}} \boldsymbol{B}_{\mathrm{dR}}, \\
&(x \otimes y) \otimes z \mapsto x \otimes(y z),
\end{aligned}
$$

et comp ${ }_{V, K_{n} / \mathbb{Q}_{p}}$ s'écrit comme le composé :

$$
\begin{aligned}
\boldsymbol{B}_{\mathrm{dR}} & \otimes_{\mathbb{Q}_{p}}\left(\operatorname{Ind}_{K / \mathbb{Q}_{p}}(V) \otimes_{\mathbb{Q}_{p}} \mathbb{Q}_{p}\left[G_{n}\right]^{l}\right) \\
& \stackrel{\sim}{\rightarrow} \boldsymbol{B}_{\mathrm{dR}} \otimes\left(\mathbb{Q}_{p}\left[G_{n}\right]^{l} \otimes_{\mathbb{Q}_{p}} \mathbb{Q}_{p}\left(\zeta_{p^{n}}\right)\right)^{G_{n}} \otimes \operatorname{Ind}_{K / \mathbb{Q}_{p}}(V) \\
& \stackrel{\sim}{\rightarrow} \boldsymbol{B}_{\mathrm{dR}} \otimes\left(\mathbb{Q}_{p}\left[G_{n}\right]^{l} \otimes_{\mathbb{Q}_{p}} \mathbb{Q}_{p}\left(\zeta_{p^{n}}\right)\right)^{G_{n}} \otimes_{\mathbb{Q}_{p}} \boldsymbol{D}_{\text {cris }}(V) \simeq \boldsymbol{B}_{\mathrm{dR}} \otimes_{\mathbb{Q}_{p}} \boldsymbol{D}_{\mathrm{dR}}^{K_{n}}(V) .
\end{aligned}
$$

L'isomorphisme $\left(\mathbb{Q}_{p}\left(\zeta_{p^{n}}\right) \otimes_{\mathbb{Q}_{p}} \mathbb{Q}_{p}\left[G_{n}\right]^{\iota}\right)^{G_{n}} \simeq \mathbb{Q}_{p}\left(\zeta_{p^{n}}\right)$ envoie $\sharp G_{n} e_{\eta}\left(x_{n}\right) \otimes e_{\eta}^{\iota}$ sur $e_{\eta}\left(x_{n}\right)$, et donc pour tous $v \in \operatorname{Ind}_{K / \mathbb{Q}_{p}}(V), \eta \in X\left(G_{n}\right)$ on a :

$$
\operatorname{comp}_{V, K_{n} / \mathbb{Q}_{p}}\left(v \otimes e_{\eta}^{\iota}\right)=\left(\operatorname{comp}_{\text {cris }}(v) e_{\eta}\left(x_{n}\right)\right) \otimes \frac{1}{\sharp G_{n} e_{\eta}\left(x_{n}\right)} .
$$

Si $\tilde{v}$ est une base de $\operatorname{det}_{\mathbb{Z}_{p}} \operatorname{Ind}_{K / \mathbb{Q}_{p}}(T)$, alors $\tilde{v}$ peut être vue comme une base de $\left.\operatorname{det}_{\mathbb{Z}_{p}\left[G_{n}\right]} \operatorname{Ind}_{K_{n} / \mathbb{Q}_{p}}(T)\right) \simeq \mathbb{Z}_{p}\left[G_{n}\right]^{l} \otimes_{\mathbb{Z}_{p}} \operatorname{det}_{\mathbb{Z}_{p}} \operatorname{Ind}_{K / \mathbb{Q}_{p}}(T)$ et pour tout $\eta \in X\left(G_{n}\right)$, on note $\tilde{v}_{\eta}=e_{\eta} \otimes \tilde{v}$ sa $\eta$-composante. Soit $\tilde{m}=\wedge m_{i}$ une base $\operatorname{de}_{\operatorname{det}} \mathbb{Z}_{p} M$. On pose

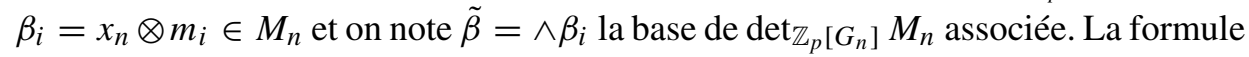
(2.1) nous donne alors :

$$
\alpha_{V, K_{n} / K, \eta}\left(\tilde{\beta}_{\eta}^{-1} \otimes \tilde{v}_{\eta}\right)=\alpha_{V, K}\left(\tilde{m}^{-1} \otimes \tilde{v}\right) \otimes\left(\frac{1}{\sharp G_{n} e_{\eta}\left(x_{n}\right)}\right)^{f d} .
$$


Si $k=a(\eta) \neq 0$, alors $e_{\eta}\left(x_{n}\right)=e_{\eta}\left(\zeta_{p^{k}}\right)$. On a une formule bien connue pour les sommes de Gauss : $\tau(\eta) \tau\left(\eta^{-1}\right)=p^{k} \eta(c)$, où $c$ est la conjugaison complexe $c: \zeta_{p^{n}} \mapsto \zeta_{p^{n}}^{-1}$ et donc $1 /\left(\sharp G_{n} e_{\eta}\left(x_{n}\right)\right)=p^{-n} \tau\left(\eta^{-1}\right) \eta(c)$.

Comme $K / \mathbb{Q}_{p}$ est non ramifiée, on a $\lambda\left(K, \mathbb{Q}_{p}\right)=1$ et la formule démontrée dans le lemme 2.16 donne :

$\beta_{V, K_{n} / K, \eta}\left(\tilde{\beta}_{\eta}^{-1} \otimes \tilde{v}_{\eta}\right)=\eta(c)^{f d} q_{K}^{-n d} \Gamma^{*}(V) \alpha_{V, K}\left(\tilde{m}^{-1} \otimes \tilde{v}\right) \operatorname{det}\left(\varphi \mid \boldsymbol{D}_{\text {cris }}(V)\right)^{-a(\eta)}$.

Si $\eta=\eta_{0}$ est le caractère trivial, alors $e_{\eta_{0}}\left(x_{n}\right)=(1-p)^{-1}$ d'où $1 /\left(\sharp G_{n} e_{\eta_{0}}\left(x_{n}\right)\right)=$ $-p^{1-n}$ et on obtient :

$$
\beta_{V, K_{n} / K, \eta_{0}}\left(\tilde{\beta}_{\eta_{0}}^{-1} \otimes \tilde{v}_{\eta_{0}}\right)=(-1)^{f d} \eta_{0}(c)^{f d} q_{K}^{(1-n) d} \Gamma^{*}(V) .
$$

Comme $\eta(c)^{f d} e_{\eta}=c^{f d} e_{\eta}$, le lemme 2.6 donne :

$$
\begin{aligned}
& \beta_{V, K_{n} / K}\left(\tilde{\beta}^{-1} \otimes \tilde{v}\right) \\
& =c^{f d} \Gamma^{*}(V) q_{K}^{-n d}\left(\sum_{\eta \neq \eta_{0}} \operatorname{det}\left(\varphi \mid \boldsymbol{D}_{\text {cris }}(V)\right)^{-a(\eta)} e_{\eta}+(-1)^{f d} q_{K}^{d} e_{\eta_{0}}\right) \alpha_{V, K}\left(\tilde{m}^{-1} \otimes \tilde{v}\right),
\end{aligned}
$$

et comme $c^{f d}$ est une unité de $\mathbb{Z}_{p}\left[G_{n}\right]$, la proposition est démontrée.

2.5. La conjecture $C_{\mathbf{E P}}(\boldsymbol{L} / K, V)$. On commence ce paragraphe par la définition de la droite d'Euler-Poincaré. Rappelons que $V$ est une représentation $p$-adique de $G_{K}$, que $L$ est une extension abélienne finie de $K$ et que l'on a posé $G=\operatorname{Gal}(L / K)$.

Définition 2.18. La droite d'Euler-Poincaré $\Delta_{\mathrm{EP}}(L / K, V)$ de $V$ est définie par la formule suivante :

$$
\begin{aligned}
\Delta_{\mathrm{EP}}(L / K, V) & =\operatorname{det}_{\mathbb{Q}_{p}[G]} \mathbb{R} \Gamma(L, V) \otimes \operatorname{det}_{\mathbb{Q}_{p}[G]}\left(\operatorname{Ind}_{L / \mathbb{Q}_{p}} V\right) \\
& \simeq \otimes_{i=0}^{2}\left(\operatorname{det}_{\mathbb{Q}_{p}[G]} H^{i}(L, V)\right)^{(-1)^{i}} \otimes \operatorname{det}_{\mathbb{Q}_{p}[G]}\left(\operatorname{Ind}_{L / \mathbb{Q}_{p}} V\right) .
\end{aligned}
$$

Si $T$ est un $\mathbb{Z}_{p}$-réseau de $V$, alors $\operatorname{Ind}_{L / \mathbb{Q}_{p}} T=\operatorname{Ind}_{K / \mathbb{Q}_{p}}\left(\mathbb{Z}_{p}[G] \otimes_{\mathbb{Z}_{p}} T\right)^{\iota}$ et $\mathbb{R} \Gamma(L, T)$ sont parfaits sur $\mathbb{Z}_{p}[G]$, et par la proposition 2.7 le sous- $\mathbb{Z}_{p}[G]$-module de $\Delta_{\mathrm{EP}}(L / K, V)$ :

$$
\Delta_{\mathrm{EP}}(L / K, T)=\operatorname{det}_{\mathbb{Z}_{p}[G]} \mathbb{R} \Gamma(L, T) \otimes \operatorname{det}_{\mathbb{Z}_{p}[G]}\left(\operatorname{Ind}_{L / \mathbb{Q}_{p}} T\right)
$$

ne dépend pas du choix de $T$ et définit donc un $\mathbb{Z}_{p}[G]$-réseau canonique de $\Delta_{\mathrm{EP}}(L / K, V)$.

Revenons aux constructions du paragraphe 1.4. Par le lemme 1.5, la suite duale de la suite (1.2) s'ecrit :

$$
\begin{aligned}
0 \rightarrow & H_{f}^{1}\left(L, V^{*}(1)\right)^{*} \rightarrow \boldsymbol{D}_{\mathrm{cris}}^{L}\left(V^{*}(1)\right)^{*} \oplus t_{V^{*}(1)}^{*}(L) \\
& \rightarrow \boldsymbol{D}_{\mathrm{cris}}^{L}\left(V^{*}(1)\right)^{*} \rightarrow H^{2}(L, V) \rightarrow 0
\end{aligned}
$$


et en composant cette suite avec la suite (1.2), on obtient une suite exacte de $\mathbb{Q}_{p}[G]$ modules :

$$
\begin{aligned}
0 \rightarrow & H^{0}(L, V) \rightarrow D_{\text {cris }}^{L}(V) \rightarrow D_{\text {cris }}^{L}(V) \oplus t_{V}(L) \rightarrow H^{1}(L, V) \\
& \rightarrow D_{\text {cris }}^{L}\left(V^{*}(1)\right)^{*} \oplus t_{V^{*}(1)}^{*}(L) \rightarrow D_{\text {cris }}^{L}\left(V^{*}(1)\right)^{*} \rightarrow H^{2}(L, V) \rightarrow 0 .
\end{aligned}
$$

En utilisant la suite exacte $0 \rightarrow t_{V^{*}(1)}^{*}(L) \rightarrow D_{\mathrm{dR}}^{L}(V) \rightarrow t_{V}(L) \rightarrow 0$, on en déduit des isomorphismes canoniques :

$$
\begin{aligned}
& \delta_{V, L / K}^{\prime}: \operatorname{det}_{\mathbb{Q}_{p}[G]} \boldsymbol{D}_{\mathrm{dR}^{L}}^{L}(V) \otimes \operatorname{det}_{\mathbb{Q}_{p}[G]} \mathbb{R} \Gamma(L, V) \stackrel{\sim}{\rightarrow} \mathbb{Q}_{p}[G], \\
& \Delta_{E P}(L / K, V) \stackrel{\sim}{\rightarrow} \operatorname{det}_{\mathbb{Q}_{p}[G]}^{-1}\left(\boldsymbol{D}_{\mathrm{dR}}^{L}(V)\right) \otimes \operatorname{det}_{\mathbb{Q}_{p}[G]}\left(\operatorname{Ind}_{L / \mathbb{Q}_{p}} V\right) .
\end{aligned}
$$

En composant le dernier isomorphisme avec l'application $\beta_{V, L / K}$, on obtient une trivialisation canonique de la droite d'Euler-Poincaré :

$$
\delta_{V, L / K}: \Delta_{E P}(L / K, V) \stackrel{\sim}{\rightarrow} \mathbb{Q}_{p}[G]_{V, L / K} .
$$

Nous pouvons maintenant enfin énoncer les conjectures $C_{\mathrm{EP}}(L / K, V)$ et $C_{\mathrm{EP}}(K, V)$ (voir [FP94], [Per95], [Kat93b]).

Conjecture $2.19\left(C_{\mathrm{EP}}(L / K, V)\right)$. Si $V=\mathbb{Q}_{p} \otimes_{\mathbb{Z}_{p}} T$ est une représentation potentiellement semi-stable de $G_{K}$ et si $L / K$ est une extension abélienne finie, alors l'application $\delta_{V, L / K}$ envoie $\Delta_{E P}(L / K, T)$ sur $\mathbb{Z}_{p}[G]_{V, L / K}$.

Si $L=K$, alors on peut reformuler cette conjecture en termes des nombres de Tamagawa locaux (voir [FP94] et la définition 1.6 ci-dessus). Soit $\omega \in \operatorname{det}_{\mathbb{Q}_{p}} \boldsymbol{D}_{\mathrm{dR}}^{K}(V)$ une base vérifiant $\omega \simeq \omega_{2}^{-1} \otimes \omega_{1}$ avec $\omega_{1} \in \operatorname{det}_{\mathbb{Q}_{p}} t_{V}(K)$ et $\omega_{2} \in \operatorname{det}_{\mathbb{Q}_{p}} t_{V^{*}(1)}(K)$. Soit $\omega_{T}$ une base de $\operatorname{Ind}_{K / \mathbb{Q}_{p}}(T)$ et soit $\alpha_{V, K}(\omega, T)=\alpha_{V, K}\left(\omega^{-1} \otimes \omega_{T}\right)$.

Conjecture 2.20 $\left(C_{\mathrm{EP}}(K, V)\right)$. Si $V=\mathbb{Q}_{p} \otimes_{\mathbb{Z}_{p}}$ T est une représentation potentiellement semi-stable de $G_{K}$, alors :

$$
\frac{\operatorname{Tam}_{K, \omega_{1}}^{0}(T)}{\operatorname{Tam}_{K, \omega_{2}}^{0}\left(T^{*}(1)\right)}=\left|d_{K}\right|_{p}^{\operatorname{dim} V / 2}\left|\Gamma^{*}(V) \frac{\alpha_{V, K}(\omega, T)}{\varepsilon(K, V)}\right|_{p} .
$$

La proposition suivante rassemble quelques propriétés fonctorielles de la conjecture $C_{\mathrm{EP}}(L / K, V)$.

Proposition 2.21. (1) Les conjectures $C_{\mathrm{EP}}(L / K, V)$ et $C_{\mathrm{EP}}\left(L / K, V^{*}(1)\right)$ sont équivalentes.

(2) Si $0 \rightarrow V^{\prime} \rightarrow V \rightarrow V^{\prime \prime} \rightarrow 0$ est une suite exacte de représentations potentiellement semi-stables et si la conjecture $C_{\mathrm{EP}}$ est vraie pour deux des représentations $V^{\prime}, V$ et $V^{\prime \prime}$, alors elle est vraie pour la troisième. 
(3) Si $M / K$ est une extension de $K$ contenue dans $L$ et si $C_{\mathrm{EP}}(L / K, V)$ est vraie, alors les conjectures $C_{\mathrm{EP}}(L / M, V)$ et $C_{\mathrm{EP}}(M / K, V)$ le sont aussi.

(4) Si la conjecture $C_{\mathrm{EP}}(L / K, V)$ est vraie, alors pour tout caractère $\eta \in X(G)$, la conjecture $C_{\mathrm{EP}}(K, V(\eta))$ est vraie.

Preuve. La démonstration se fait comme dans [Per95, C.2.9], en utilisant en plus les remarques suivantes :

(1) La dualité locale donne un isomorphisme $\operatorname{det}_{\mathbb{Z}_{p}[G]} \mathbb{R H o m}_{\mathbb{Z}_{p}}\left(\mathbb{R} \Gamma(L, T), \mathbb{Z}_{p}\right) \simeq$ $\operatorname{det}_{\mathbb{Z}_{p}[G]} \mathbb{R} \Gamma\left(L, T^{*}(1)\right)$.

(2) Pour le triangle exact $\mathbb{R} \Gamma\left(L, T^{\prime}\right) \rightarrow \mathbb{R} \Gamma(L, T) \rightarrow \mathbb{R} \Gamma\left(L, T^{\prime \prime}\right) \rightarrow \mathbb{R} \Gamma\left(L, T^{\prime}\right)[1]$, on a un isomorphisme fonctoriel $\operatorname{det}_{\mathbb{Z}_{p}[G]} \mathbb{R} \Gamma(L, T) \stackrel{\sim}{\rightarrow} \operatorname{det}_{\mathbb{Z}_{p}[G]} \mathbb{R} \Gamma\left(L, T^{\prime}\right) \otimes$ $\operatorname{det}_{\mathbb{Z}_{p}[G]} \mathbb{R} \Gamma\left(L, T^{\prime \prime}\right)$ (voir [KM76, proposition 7]).

(3) Si on pose

$$
H=\operatorname{Gal}(L / M), \quad D_{L / M}=\boldsymbol{D}_{\mathrm{pst}}\left(\operatorname{Ind}_{L / M} V\right), \quad D_{L / K}=\boldsymbol{D}_{\mathrm{pst}}\left(\operatorname{Ind}_{L / K} V\right),
$$

alors pour tout $\eta \in X(H)$ on a :

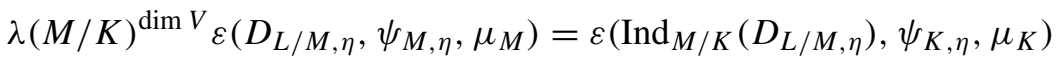

$$
\begin{aligned}
& =\prod_{\substack{\hat{\eta} \in X(G) \\
\hat{\eta} \mapsto \eta}} \varepsilon\left(D_{L / K, \hat{\eta}}, \psi_{\hat{\eta}}, \mu_{K}\right) .
\end{aligned}
$$

On en déduit que la restriction transforme $\beta_{V, L / K}$ en $\beta_{V, L / M}$ et le fait que $C_{\mathrm{EP}}(L / K, V)$ implique $C_{\mathrm{EP}}(L / M, V)$ résulte maintenant de la proposition 2.1. La deuxième implication est analogue : on voit facilement que la projection de $\mathbb{Q}_{p}[G] \operatorname{sur} \mathbb{Q}_{p}[G / H]$ transforme $\beta_{V, L / K}$ en $\beta_{V, M / K}$.

(4) Soit $E$ un corps contenant toutes les valeurs des caractères $\eta \in X(G)$ et soit $V(\eta)=E(\eta) \otimes_{\mathbb{Q}_{p}} V$. Si on note $\mathcal{A}(G)$ l'ordre maximal de $E[G]$, alors on a des isomorphismes canoniques :

$\mathcal{A}(G) \otimes_{\mathbb{Z}_{p}[G]}^{L} \mathbb{R} \Gamma(L, T) \stackrel{\sim}{\rightarrow} \mathbb{R} \Gamma\left(K, \mathcal{A}(G) \otimes_{\mathbb{Z}_{p}} T\right) \stackrel{\sim}{\rightarrow} \bigoplus_{\eta \in X(G)} \mathbb{R} \Gamma(K, T(\eta))$.

Si la conjecture $C_{\mathrm{EP}}(L / K, V)$ est vraie, alors l'application $\delta_{V, L / K}$ envoie $\Delta_{E P}\left(K, \mathcal{A}(G) \otimes_{\mathbb{Z}_{p}} T\right)$ sur $\mathcal{A}(G)_{V, L / K}=\mathcal{A}(G) \otimes_{\mathbb{Z}_{p}[G]} \mathbb{Z}_{p}[G]_{V, L / K}$. En décomposant cet isomorphisme caractère par caractère, on en déduit les conjectures $C_{\mathrm{EP}}(K, V(\eta))$ pour tous les caractères $\eta \in X(G)$. 


\section{L'exponentielle de Perrin-Riou}

Dans tout ce chapitre, on suppose que $K$ est une extension non-ramifiée de $\mathbb{Q}_{p}$. On commence par des rappels et des compléments sur l'exponentielle de Perrin-Riou, ce qui nous permet d'énoncer la conjecture $C_{\mathrm{Iw}}\left(K_{\infty} / K, V\right)$. Dans le chapitre suivant, on montre que $C_{\mathrm{Iw}}\left(K_{\infty} / K, V\right)$ est équivalente à $C_{\mathrm{EP}}\left(K_{n} / K, V\right)$ pour tout $n \geqslant 1$ et finalement, on démontre la conjecture $C_{\mathrm{Iw}}\left(K_{\infty} / K, V\right)$.

3.1. Rappels et compléments. L'objet de ce paragraphe est de rappeler la construction et certaines propriétés de l'exponentielle de Perrin-Riou, tout d'abord telle qu' elle a été définie par Perrin-Riou elle-même dans [Per94], puis ensuite (dans le paragraphe suivant) telle qu'elle a été faite par l'un d'entre nous dans [Ben00].

Rappelons que $K_{n}=K\left(\zeta_{p^{n}}\right)$, que $K_{\infty}=\bigcup_{n \geqslant 1} K_{n}$, que $\Gamma=\operatorname{Gal}\left(K_{\infty} / K\right)$ et que $G_{n}=\operatorname{Gal}\left(K_{n} / K\right) \simeq \Gamma / \Gamma_{n}$. On fixe un générateur topologique $\gamma_{1}$ de $\Gamma_{1}$ et on pose $\gamma_{n}=\gamma_{1}^{p^{n-1}}$ ce qui fait de $\gamma_{n}$ un générateur topologique de $\Gamma_{n}$. Si on note $\Delta_{K}$ le sous-groupe de torsion de $\Gamma$, alors on a $\Gamma \simeq \Delta_{K} \times \Gamma_{1}$ et $\Lambda=\mathbb{Z}_{p}\left[\Delta_{K}\right] \otimes_{\mathbb{Z}_{p}} \mathbb{Z}_{p}\left[\left[\Gamma_{1}\right]\right]$. On définit une action de $\Gamma$ sur $K[[X]]$ par la formule :

$$
g(X)=(1+X)^{\chi(g)}-1,
$$

où $\chi: \Gamma \rightarrow \mathbb{Z}_{p}^{\times}$est le caractère cyclotomique. On munit par ailleurs $K[[X]]$ d'un Frobenius $\varphi$ et d'un opérateur différentiel $\partial$ en posant :

$$
\begin{aligned}
\varphi\left(\sum_{i=0}^{+\infty} a_{i} X^{i}\right) & =\sum_{i=0}^{+\infty} a_{i}^{\sigma} \varphi(X)^{i}, \quad \text { où } \varphi(X)=(1+X)^{p}-1, \\
\partial & =(1+X) \frac{d}{d X} .
\end{aligned}
$$

On vérifie facilement que $\partial \circ \varphi=p \varphi \circ \partial$. Soit $\psi: K[[X]] \rightarrow K[[X]]$ l'opérateur défini par la formule :

$$
\psi(f(X))=\frac{1}{p} \varphi^{-1}\left(\sum_{\zeta^{p}=1} f(\zeta(1+X)-1)\right),
$$

qui est compatible avec la définition du paragraphe 1.3. Il est classique que $\mathcal{O}_{K}[[X]]^{\psi=0}=\left\{f \in \mathcal{O}_{K}[[X]] \mid \psi(f)=0\right\}$ est un $\mathcal{O}_{K}[[\Gamma]]$-module libre engendré par $1+X$.

On note $\mathscr{H}$ l'ensemble des séries formelles $f(X) \in \mathbb{Q}_{p}[[X]]$ qui convergent sur le disque unité ouvert, c'est-à-dire $\left\{x \in \mathbb{C}_{p},|x|_{p}<1\right\}$, et l'on pose $\mathscr{H}\left(\Gamma_{1}\right)=$ $\left\{f\left(\gamma_{1}-1\right), f \in \mathscr{H}\right\}$ et $\mathcal{H}(\Gamma)=\mathbb{Q}_{p}\left[\Delta_{K}\right] \otimes_{\mathbb{Q}_{p}} \mathcal{H}\left(\Gamma_{1}\right)$. Pour tout $\Lambda$-module $N$, l'homomorphisme naturel $N \rightarrow N_{\Gamma_{n}}$ se prolonge en une application $\mathscr{H}(\Gamma) \otimes_{\Lambda} N \rightarrow$ $\mathbb{Q}_{p} \otimes_{\mathbb{Z}_{p}} N_{\Gamma_{n}}$ 
Si $V$ est une représentation cristalline de $G_{K}$, alors on pose

$$
\mathscr{D}(V)=\mathcal{O}_{K}[[X]]^{\psi=0} \otimes_{\mathcal{O}_{K}} \boldsymbol{D}_{\text {cris }}(V) .
$$

Pour tout $k \in \mathbb{Z}$, on définit une application $\Delta_{k}: \mathscr{D}(V) \rightarrow \boldsymbol{D}_{\text {cris }}(V) /\left(1-p^{k} \varphi\right) \boldsymbol{D}_{\text {cris }}(V)$ par la formule $\Delta_{k}(f)=\left(\partial^{k} f\right)(0) \bmod \left(1-p^{k} \varphi\right) \boldsymbol{D}_{\text {cris }}(V)$. Si on écrit $\Delta=$ $\bigoplus_{k \in \mathbb{Z}} \Delta_{k}$, alors pour tout $f \in \mathscr{D}(V)^{\Delta=0}$, l'équation $(1-\varphi) F(X)=f(X)$ a une solution dans $\mathscr{H}(V)=\mathscr{H} \otimes_{\mathbb{Q}_{p}} \boldsymbol{D}_{\text {cris }}(V)$ et on en déduit une application :

$$
\begin{aligned}
\Xi_{V, n}^{\varepsilon}: \mathscr{D}(V)^{\Delta=0} & \rightarrow \boldsymbol{D}_{\mathrm{dR}}^{K_{n}}(V) / \boldsymbol{D}_{\text {cris }}(V)^{\varphi=1}, \\
f & \mapsto p^{-n}(\sigma \otimes \varphi)^{-n}(F)\left(\zeta_{p^{n}}-1\right) .
\end{aligned}
$$

Dans [Per94], Perrin-Riou a démontré le résultat suivant.

Théorème 3.1. Si h est un entier tel que $\mathrm{Fil}^{-h} \boldsymbol{D}_{\mathrm{dR}}^{K}(V)=D_{\mathrm{dR}}^{K}(V)$, alors pour tout $i \in \mathbb{Z}$ vérifiant $i+h \geqslant 1$, il existe un $\Lambda$-homomorphisme (appelé exponentielle élargie, ou exponentielle de Perrin-Riou) :

$$
\operatorname{Exp}_{V(i), h+i}^{\varepsilon}: \mathscr{D}(V(i))^{\Delta=0} \rightarrow \mathscr{H}(\Gamma) \otimes_{\Lambda}\left(H_{\mathrm{Iw}}^{1}(K, T(i)) / T(i)^{H_{K}}\right),
$$

vérifiant les propriétés suivantes:

(1) Le diagramme ci-dessous est commutatif:

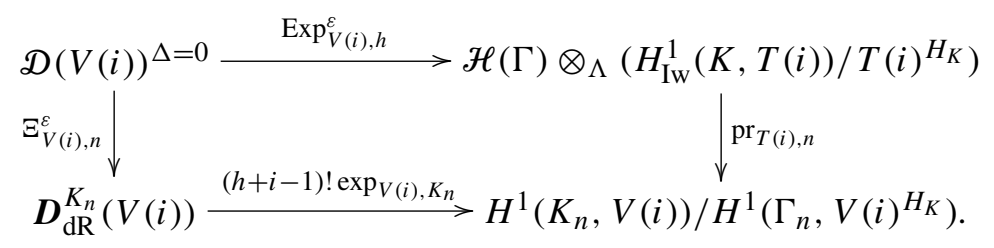

(2) Soit $e_{1}=\varepsilon^{-1} \otimes t$ le générateur de $\boldsymbol{D}_{\text {cris }}\left(\mathbb{Q}_{p}(-1)\right)$ associé au choix de $\varepsilon$ et soit :

$$
\mathrm{Tw}_{V(i), k}^{\varepsilon}: H_{\mathrm{Iw}}^{1}(K, V(i)) \rightarrow H_{\mathrm{Iw}}^{1}(K, V(i+k))
$$

l'application définie par $\operatorname{Tw}_{V(i), k}^{\varepsilon}(x)=x \otimes \varepsilon^{\otimes k}$. On a alors :

$$
\operatorname{Exp}_{V(i+1), h+1}^{\varepsilon}=-\operatorname{Tw}_{V(i), 1}^{\varepsilon} \circ \operatorname{Exp}_{V(i), h}^{\varepsilon} \circ\left(\partial \otimes e_{1}\right) .
$$

(3) Si :

$$
\ell_{m}=m-\frac{\log \left(\gamma_{1}\right)}{\log \chi\left(\gamma_{1}\right)},
$$

alors $\operatorname{Exp}_{V(i), h+1}^{\varepsilon}=\ell_{h} \operatorname{Exp}_{V(i), h}^{\varepsilon}$ 
On déduit de ce théorème plusieurs formules qui nous sont utiles. Tout d'abord, en itérant (2), on obtient :

$$
\operatorname{Exp}_{V(i), h+i}^{\varepsilon}=(-1)^{i} \mathrm{Tw}_{V, i}^{\varepsilon} \circ \operatorname{Exp}_{V, h}^{\varepsilon} \circ\left(\partial^{i} \otimes e_{i}\right) .
$$

Soit $\mathcal{K}(\Gamma)$ l'anneau total des fractions de $\mathscr{H}(\Gamma)$ (il suffit en fait d'inverser les $\ell_{i}$ ). Le (3) permet de définir pour tout $h \in \mathbb{Z}:$

$$
\operatorname{Exp}_{V, h}^{\varepsilon}: \mathscr{D}(V)^{\Delta=0} \rightarrow \mathcal{K}(\Gamma) \otimes_{\Lambda}\left(H_{\mathrm{Iw}}^{1}(K, T) / T^{H_{K}}\right) .
$$

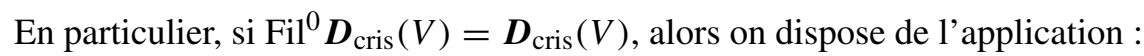

$$
\operatorname{Exp}_{V, 0}^{\varepsilon}: \mathscr{D}(V)^{\Delta=0} \rightarrow \mathscr{H}(\Gamma) \otimes_{\Lambda}\left(H_{\mathrm{IW}}^{1}(K, T) / T^{H_{K}}\right),
$$

qui est telle que pour tout $i \geqslant 1$, si l'on pose $\Xi_{V, n}^{(i), \varepsilon}=\Xi_{V(i), n}^{\varepsilon} \circ\left(\partial^{-i} \otimes e_{-i}\right)$, alors le diagramme ci-dessous commute :

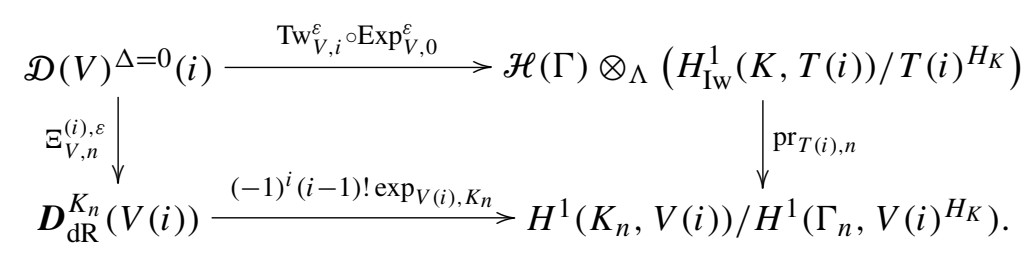

Rappelons à présent quelques résultats techniques concernant l'application $\Xi_{V, n}^{\varepsilon}$ et qui sont démontrés dans [Per94, §3.4]. L'homomorphisme $\Delta$ donne lieu à une suite exacte courte :

$$
0 \rightarrow \mathscr{D}(V)^{\Delta=0} \rightarrow \mathscr{D}(V) \stackrel{\Delta}{\rightarrow} \bigoplus_{j \in \mathbb{Z}}\left(\frac{\boldsymbol{D}_{\text {cris }}(V)}{\left(1-p^{j} \varphi\right) \boldsymbol{D}_{\text {cris }}(V)}\right)(j) \rightarrow 0,
$$

qui induit une suite exacte :

$$
0 \rightarrow \frac{\boldsymbol{D}_{\text {cris }}(V)}{(1-\varphi) \boldsymbol{D}_{\text {cris }}(V)} \rightarrow\left(\mathscr{D}(V)^{\Delta=0}\right)_{\Gamma_{n}} \rightarrow \mathscr{D}(V)_{\Gamma_{n}} \rightarrow \frac{\boldsymbol{D}_{\text {cris }}(V)}{(1-\varphi) \boldsymbol{D}_{\text {cris }}(V)} \rightarrow 0 .
$$

La deuxième flèche de cette suite est donnée par la formule $\tilde{d} \mapsto d \otimes\left(\gamma_{n}-1\right)(1+X)$ si $\tilde{d}=d \bmod (1-\varphi) \boldsymbol{D}_{\text {cris }}(V)$.

L'application $\Xi_{V, n}^{\varepsilon}$ se factorise par $\left(\gamma_{n}-1\right) \mathscr{D}(V)^{\Delta=0}$ et on note :

$$
\tilde{\Xi}_{V, n}^{\varepsilon}:\left(\mathcal{D}(V)^{\Delta=0}\right)_{\Gamma_{n}} \rightarrow \boldsymbol{D}_{\mathrm{dR}}^{K_{n}}(V) / \boldsymbol{D}_{\text {cris }}(V)^{\varphi=1}
$$

la flèche qui s'en déduit. Soit :

$$
\operatorname{Exp}_{V, h, n}^{\varepsilon}:\left(\mathcal{D}(V)^{\Delta=0}\right)_{\Gamma_{n}} \rightarrow\left(\mathbb{Q}_{p} \otimes_{\mathbb{Z}_{p}} H_{\mathrm{IW}}^{1}(K, T) / T^{H_{K}}\right)_{\Gamma_{n}}
$$

l'application déduite de $\operatorname{Exp}_{V, h}^{\varepsilon}$. 
Proposition 3.2. (1) La suite :

$$
0 \rightarrow \boldsymbol{D}_{\text {cris }}(V) /(1-\varphi) \boldsymbol{D}_{\text {cris }}(V) \rightarrow \operatorname{ker} \tilde{\boldsymbol{\Xi}}_{V, n}^{\varepsilon} \stackrel{f}{\rightarrow} \boldsymbol{D}_{\text {cris }}(V)^{\varphi=p^{-1}} \rightarrow 0,
$$

où $f(\alpha(X))=\alpha(0)$, est exacte.

(2) L'application $\alpha \mapsto \operatorname{Tr}_{K_{n} / K}(\alpha)$ induit un isomorphisme :

$$
\operatorname{coker}\left(\tilde{\Xi}_{V, n}^{\varepsilon}\right) \stackrel{\sim}{\rightarrow} \boldsymbol{D}_{\text {cris }}(V) /\left(1-p^{-1} \varphi^{-1}\right) \boldsymbol{D}_{\text {cris }}(V) .
$$

(3) On a une suite exacte :

$$
\begin{aligned}
0 \rightarrow & \operatorname{ker}\left(\tilde{\Xi}_{V, n}^{\varepsilon}\right) \rightarrow \operatorname{ker}\left(\operatorname{Exp}_{V, h, n}^{\varepsilon}\right) \stackrel{\tilde{\Xi}_{V, n}^{\varepsilon}}{\longrightarrow} \operatorname{Fil}^{0} \boldsymbol{D}_{\mathrm{dR}}^{K_{n}}(V) / V^{G_{K}} \\
& \rightarrow \boldsymbol{D}_{\text {cris }}(V) /\left(1-p^{-1} \varphi^{-1}\right) \boldsymbol{D}_{\text {cris }}(V) \rightarrow\left(V^{*}(1)^{G_{K}}\right)^{*} \rightarrow 0,
\end{aligned}
$$

dont les trois derniers termes sont obtenus en dualisant la suite exacte (1.1) (voir le lemme 1.5).

Preuve. Voir [Per94, 3.4.4-3.4.5]. Remarquons néanmoins que dans [Per94], PerrinRiou utilise une autre normalisation de l'isomorphisme (2), à savoir $\alpha \mapsto(1-\varphi)$ $\operatorname{Tr}_{K_{n} / K}(\alpha)\left(\bmod \left(1-p^{-1} \varphi^{-1}\right) \boldsymbol{D}_{\text {cris }}(V)\right)$. Comme l'opérateur $1-\varphi$ coïncide avec la multiplication par $1-1 / p$ sur $\left(1-p^{-1} \varphi^{-1}\right) \boldsymbol{D}_{\text {cris }}(V)$, cela ne change pas les énoncés. Le choix que nous faisons dans ce texte semble plus naturel (voir le paragraphe 4.3).

3.2. L'application exponentielle et les $(\varphi, \Gamma)$-modules. Nous rappelons maintenant la construction de l'exponentielle en termes de $(\varphi, \Gamma)$-modules qu' a donnée l'un d'entre nous (dans [Ben00]). On suppose désormais que $V$ est une représentation cristalline de $G_{K}$ qui est positive, c'est-à-dire que les opposés des poids de HodgeTate de $V$ sont $0=r_{1} \leqslant r_{2} \leqslant \cdots \leqslant r_{d}=h$. On fixe un $\mathbb{Z}_{p}$-réseau $T$ de $V$ stable par $G_{K}$, et on définit un $\mathcal{O}_{K}$-réseau $M$ de $\boldsymbol{D}_{\text {cris }}(V)$ par :

$$
M=\left\{f(X) \in\left(B_{\mathrm{rig}, K}^{+} \otimes_{A_{K}^{+}} N(T)\right)^{\Gamma} \mid f(0) \in N(T) / X N(T)\right\} .
$$

La proposition V.1 de [Ber04] nous dit que le déterminant de l'isomorphisme de comparaison $\boldsymbol{B}_{\mathrm{dR}} \otimes_{\mathbb{Q}_{p}} \operatorname{Ind}_{K / \mathbb{Q}_{p}} V \simeq \boldsymbol{B}_{\mathrm{dR}} \otimes_{\mathbb{Q}_{p}} \boldsymbol{D}_{\text {cris }}(V)$, calculé dans des bases de $T$ et de $M$, appartient à $\hat{\mathcal{O}}_{K^{\text {nr }}}^{\times} t^{r_{1}+\cdots+r_{d}}$, c'est-à-dire que dans les notations de la section 2.4, on a $\alpha_{V, K}(M, T) \in \hat{\mathcal{O}}_{K^{\mathrm{nr}}}^{\times}$.

L'anneau $\mathcal{O}_{K}[[X]]$ est muni comme ci-dessus des opérateurs $\psi$ et $\partial=(1+$ $X) d / d X$, et on pose $\mathscr{D}(T)=\mathcal{O}_{K}[[X]]^{\psi=0} \otimes_{\mathcal{O}_{K}} M$, où $M$ est le réseau de $\boldsymbol{D}_{\text {cris }}(V)$ que l'on vient de définir.

Pour des raisons techniques, on remplace le complexe $C_{\varphi, \gamma_{n}}\left(K_{n}, T\right)$ par le complexe $\varphi^{-n}\left(C_{\varphi, \gamma_{n}}\left(K_{n}, T\right)\right)$ de $(\varphi, \Gamma)$-modules sur $\boldsymbol{A}_{K_{n}}=\varphi^{-n}\left(\boldsymbol{A}_{K}\right)$, complexe qui est isomorphe à $C_{\varphi, \gamma_{n}}\left(K_{n}, T\right)$. On pose $X_{n}=\left[\varepsilon^{1 / p^{n}}\right]-1$. 
Supposons d'abord que $V^{H_{K}}=0$ et rappelons que dans cet article, $p \neq 2$. Soit $n_{1}, \ldots, n_{d}$ une base de $N(T)$ et soit $m=\sum_{i=1}^{d} a_{i}(X) \otimes n_{i}$, avec $a_{i}(X) \in$ $\boldsymbol{B}_{\text {rig, } K}^{+}$, un élément de $M$. Si $\gamma \in \Gamma$, alors un petit calcul qui utilise les congruences $\gamma\left(n_{i}\right) \equiv n_{i} \bmod X \boldsymbol{N}(T)$ montre que si l'on pose $c_{k}=\prod_{j=1}^{k}\left(\chi^{j}(\gamma)-1\right)$ pour un générateur topologique $\gamma$ de $\Gamma$ et pour tout $k \geqslant 1$, alors $a_{i}(X)$ appartiennent à l'anneau $\boldsymbol{A}_{K}^{\prime}=\boldsymbol{A}_{K}^{+}\left[\left[X^{k} / c_{k}, k \geqslant 0\right]\right]$. Si $\alpha=f(x) \otimes m \in \mathscr{D}(T)$, alors on pose $E_{k, n}(f) \otimes m=\sum_{i=1}^{d}\left(a_{i}(X) E_{k, n}(f)\right) \otimes n_{i}$, où :

$$
E_{k, n}(f)=\sum_{j=1}^{\infty} \frac{(1-k)(2-k) \ldots(j-k-1)}{t^{j}} p^{n(j-1)} \partial^{-j}\left(f\left(X_{n}\right)\right) .
$$

On tronque les séries $a_{i}(X) / t^{j}$ modulo $X$ et on note $\overline{a_{i} E_{k, n}(f)}$ les séries que l'on obtient ainsi. Soit :

$$
\mathcal{E}_{T, k, n}(\alpha)=\sum_{i=1}^{d} n_{i} \otimes \overline{a_{i} E_{k, n}(f)} \otimes \varepsilon^{\otimes k} .
$$

On vérifie que $\mathcal{E}_{T, k, n}(\alpha) \in \varphi^{-n}(\boldsymbol{D}(T(k)))$ et on définit $\mathcal{F}_{T, k, n}(\alpha) \in \varphi^{-n}(\boldsymbol{D}(T(k)))$ par :

$$
(1-\varphi) \mathcal{F}_{T, k, n}(\alpha)=\left(1-\gamma_{n}\right) \mathcal{E}_{T, k, n}(\alpha),
$$

ce qui fait que $\left(\mathcal{E}_{T, k, n}(\alpha), \mathcal{F}_{T, k, n}(\alpha)\right)$ définit une classe de cohomologie dans l'espace $H^{1}\left(\varphi^{-n}\left(C_{\varphi, \gamma_{n}}\left(K_{n}, T(k)\right)\right)\right)$. En composant avec l'isomorphisme :

$$
H^{1}\left(\varphi^{-n}\left(C_{\varphi, \gamma_{n}}\left(K_{n}, T(k)\right)\right)\right) \stackrel{\sim}{\rightarrow} H^{1}\left(K_{n}, T(k)\right),
$$

on obtient un homomorphisme :

$$
\Omega_{T, k, n}^{\varepsilon}: \mathscr{D}(T) \rightarrow H^{1}\left(K_{n}, T(k)\right) .
$$

Revenons maintenant au cas général (on ne suppose plus que $V^{H_{K}}=0$ ) et posons :

$$
\mathscr{H}(T)=\left\{\alpha \in \mathcal{O}_{K}[[X]] \otimes_{\mathcal{O}_{K}} M \mid \psi(\alpha)=\alpha\right\} .
$$

Rappelons que pour tout $j \in \mathbb{Z}$ on a défini un homomorphisme $\Delta_{j}: \mathscr{D}(V) \rightarrow$ $\boldsymbol{D}_{\text {cris }}(V) /\left(1-p^{j} \varphi\right) \boldsymbol{D}_{\text {cris }}(V)$ par la formule

$$
\Delta_{j}(f)=\partial^{j} f(0) \quad\left(\bmod \left(1-p^{j} \varphi\right) \boldsymbol{D}_{\text {cris }}(V)\right) .
$$

Un petit calcul montre qu'on a une suite exacte :

$$
0 \rightarrow M^{\varphi=1} \rightarrow \mathcal{H}(T) \stackrel{1-\varphi}{\longrightarrow} \mathscr{D}(T)^{\Delta_{0}=0} \rightarrow 0,
$$


(voir par exemple [Ben00, §§4.1.2 - 4.1.3]) et la même construction qu'avant fournit un homomorphisme :

$$
\Sigma_{T, k, n}^{\varepsilon}: \mathscr{H}(T) \rightarrow H^{1}\left(K_{n}, T(k)\right)
$$

qui s'inscrit dans un diagramme :

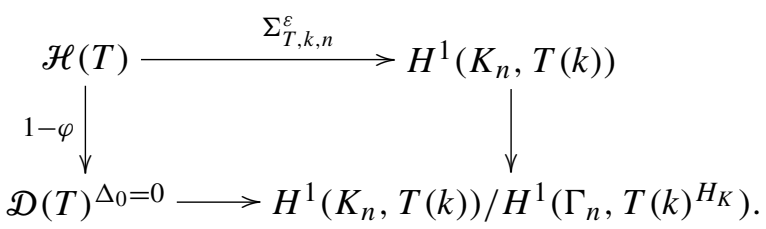

En particulier, si $V^{H_{K}}=0$, alors on a :

$$
\Sigma_{T, k, n}^{\varepsilon}(\alpha)=\Omega_{T, k, n}^{\varepsilon}((1-\varphi) \alpha) .
$$

Les résultats suivants sont démontrés dans [Ben00, théorèmes 4.3 et 5.1.2].

Proposition 3.3. Si $V$ est une représentation positive vérifiant $V^{H_{K}}=0$ et si $\alpha \in$ $\mathscr{D}(T)$, alors :

(1) Pour tous $k \geqslant 1$ et $n \geqslant 1$, on $a$ :

$$
\Omega_{T, k, n}^{\varepsilon}(\alpha)=(-1)^{k}(k-1) ! \exp _{V(k), K_{n}}\left(F_{k}\left(\zeta_{p^{n}}-1\right)\right)
$$

où $F_{k}(X)$ est une solution de l'équation $(1-\varphi) F_{k}=\left(\partial^{-k} \otimes e_{-k}\right)(\alpha)$ et $e_{-k}$ est le générateur de $\boldsymbol{D}_{\text {cris }}\left(\mathbb{Q}_{p}(k)\right)$ associé à $\varepsilon$.

(2) Plus généralement, pour tous $k \in \mathbb{Z}$ et $n \geqslant 1$, on $a:$

$$
\Omega_{T, k, n}^{\varepsilon}\left((\varphi \otimes \sigma)^{-n}(\alpha)\right)=\operatorname{pr}_{V(k), n} \circ \operatorname{Tw}_{V, k}^{\varepsilon} \circ \operatorname{Exp}_{V, 0}^{\varepsilon}(\alpha) .
$$

(3) Soit $(\cdot, \cdot)_{T(k), n}: H^{1}\left(K_{n}, T(k)\right) \times H^{1}\left(K_{n}, T^{*}(1-k)\right) \stackrel{\cup}{\rightarrow} \mathbb{Q}_{p}$ l'accouplement fourni par la dualité locale. On a alors

$$
\begin{aligned}
& \left(\Omega_{T, k, n}^{\varepsilon}(\alpha), \Omega_{T^{*}(-h), h-k+1, n}^{\varepsilon}(\beta)\right)_{T(k), n}= \\
& (-1)^{k} p^{n h} \prod_{m=1}^{h}(k-m) \operatorname{Tr}_{K / \mathbb{Q}_{p}} \operatorname{res}\left(\frac{1}{X}\left[\partial^{-k} \alpha\left(X_{n}\right),\left(\partial^{k-h-1} \otimes e_{-h}\right) \beta\left(X_{n}\right)\right] \frac{d X_{n}}{1+X_{n}}\right) .
\end{aligned}
$$

Remarque 3.4. (1) Cette proposition entraîne la loi de réciprocité de Perrin-Riou (le théorème 4.1 ci-dessous);

(2) rappelons qu'une représentation cristalline $W$ telle que $W=W^{H_{K}}$ est nécessairement de la forme $W=\bigoplus_{i \in \mathbb{Z}} \mathbb{Q}_{p}(i)^{d_{i}}$, voir [Per94, lemme 3.4.3]. 
Proposition 3.5. Si $V$ n'a pas de sous-quotient isomorphe à $\mathbb{Q}_{p}(m)$, avec $m \in \mathbb{Z}$, alors pour tout $k \notin[1, h]$, l'application $\Omega_{T, k, 1}^{\varepsilon}$ induit un isomorphisme

$$
\mathscr{D}(T)(k)_{\Gamma_{1}} \stackrel{\sim}{\rightarrow}\left(\varphi^{*} N(T)(k)\right)_{\Gamma_{1}}^{\psi=1} \hookrightarrow H_{\mathrm{IW}}^{1}(K, T(k))_{\Gamma_{1}} .
$$

La démonstration de cette proposition fait l'objet du reste de ce paragraphe. Pour simplifier la notation on pose $\Omega_{T, k}^{\varepsilon}=\Omega_{T, k, 1}^{\varepsilon}$.

Lemme 3.6. Le $\Lambda$-module $\left(\varphi^{*} \boldsymbol{N}(T)\right)^{\psi=1}$ est libre de rang $d=\operatorname{dim}(V)$ et pour tout $k \notin[1, h]$, l'application $\Omega_{T, k}^{\varepsilon}$ induit une injection :

$$
\mathscr{D}(T)(k)_{\Gamma_{1}} \stackrel{\Omega_{T, k}^{\varepsilon}}{\hookrightarrow}\left(\varphi^{*} N(T)(k)\right)_{\Gamma_{1}}^{\psi=1} \hookrightarrow H_{\mathrm{Iw}}^{1}(K, T(k))_{\Gamma_{1}} .
$$

Preuve. Rappelons que $\boldsymbol{A} \subset W(\boldsymbol{E})$ et soit $\boldsymbol{A}^{>0}$ l'ensemble des $x=\sum_{k=0}^{\infty} p^{k}\left[x_{k}\right] \epsilon$ $\boldsymbol{A}$ tels que $x_{k} \in \mathfrak{m}_{\boldsymbol{E}}$ pour tout $k \geqslant 0$. On a une suite exacte scindée $0 \rightarrow \boldsymbol{A}^{>0} \rightarrow$ $\boldsymbol{A}^{+} \leftrightarrows W\left(\bar{k}_{K}\right) \rightarrow 0$. Si $\boldsymbol{D}^{>0}(T)=\left(\boldsymbol{A}^{>0} \otimes_{\mathbb{Z}_{p}} T\right)^{H_{K}}$, alors on a une suite exacte :

$$
0 \rightarrow \boldsymbol{D}^{>0}(T) \rightarrow \boldsymbol{D}^{+}(T) \leftrightarrows\left(W\left(\bar{k}_{K}\right) \otimes_{\mathbb{Z}_{p}} T\right)^{H_{K}} \rightarrow 0
$$

Comme la restriction de $\psi$ à $W\left(\bar{k}_{K}\right)$ coïncide avec $\varphi^{-1}$, et que par hypothèse $T^{H_{K}}=0$, on a $\left(\left(W\left(\bar{k}_{K}\right) \otimes_{\mathbb{Z}_{p}} T\right)^{\psi=1}\right)^{H_{K}}=0$, d'où $\boldsymbol{D}^{+}(T)^{\psi=1}=\boldsymbol{D}^{>0}(T)^{\psi=1}$. Comme $T$ est positive, on a $\varphi^{*}(N(T)) \subset N(T) \subset D^{+}(T)$, d'où $\left(\varphi^{*} N(T)\right)^{\psi=1} \subset$ $\boldsymbol{D}^{>0}(T)^{\psi=1}$. L'opérateur $1-\varphi$ est inversible sur $\boldsymbol{D}^{>0}(T)$, d'inverse $\sum_{j \geqslant 0} \varphi^{j}$, et un petit calcul montre qu'il donne lieu à un isomorphisme :

$$
\left(\varphi^{*} \boldsymbol{N}(T)\right)^{\psi=1} \stackrel{\sim}{\rightarrow}\left(\varphi^{*} N(T)\right)^{\psi=0} .
$$

Pour montrer que le $\Lambda$-module $\left(\varphi^{*} N(T)\right)^{\psi=1}$ est libre de rang $d=\operatorname{dim} V$, il reste enfin à remarquer que $\left(\varphi^{*} \boldsymbol{N}(T)\right)^{\psi=0}$ est un $\Lambda$-module libre engendré par les éléments $\varphi\left(n_{i}\right) \otimes(1+X)$, où $n_{1}, \ldots, n_{d}$ est une base de $N(T)$.

Posons maintenant $\alpha=f \otimes m \in \mathscr{D}(T)$, où $m=\sum_{i=1}^{d} a_{i}(X) \otimes n_{i} \in\left(\boldsymbol{B}_{\text {rig, },}^{+} \otimes_{A_{K}^{+}}\right.$ $\boldsymbol{N}(T))^{\Gamma}$ et $f \in \mathcal{O}_{K}[[X]]^{\psi=0}$. La proposition 3.1.3 de [Ben00] montre que l'on a alors :

$$
\begin{aligned}
\left(1-\gamma_{1}\right) \mathcal{E}_{T, k}(\alpha) & \equiv\left(1-\gamma_{1}\right)\left(E_{k, 1}(f) \otimes m \otimes \varepsilon^{\otimes k}\right) \\
& \equiv \frac{1-\chi\left(\gamma_{1}\right)^{k}}{p^{k}} f\left(X_{1}\right) \otimes m \otimes \varepsilon^{\otimes k} \bmod X_{1} \mathbb{Q}_{p}\left[\left[X_{1}\right]\right] \otimes_{A_{K}^{+}} N(T)(k) \\
& \equiv \sum_{i=1}^{d} a_{i}(0) \frac{1-\chi\left(\gamma_{1}\right)^{k}}{p^{k}} f\left(X_{1}\right) \otimes n_{i} \otimes \varepsilon^{\otimes k} \bmod X_{1} N(T)(k)
\end{aligned}
$$


ce qui fait que $\left(1-\gamma_{1}\right) \mathcal{E}_{T, k}(\alpha) \in \boldsymbol{A}_{K_{1}} \otimes_{A_{K_{1}}^{+}} \boldsymbol{N}(T)(k)$. D'autre part, soit $\psi_{1}$ l'opérateur $\psi$ agissant sur $\boldsymbol{A}_{K_{1}}$. Comme $\psi_{1}\left(\partial^{-j} f\left(X_{1}\right)\right)=0$ et $\psi_{1}\left(X^{m} x\right)=X_{1}^{m} \psi_{1}(x)$, on a $\psi_{1}\left(\mathcal{E}_{T, k}(\alpha)\right)=0$ et donc $\left(1-\gamma_{1}\right) \mathcal{E}_{T, k}(\alpha) \in\left(\boldsymbol{A}_{K_{1}} \otimes_{A_{K_{1}}^{+}} \boldsymbol{N}(T)(k)\right)^{\psi_{1}=0}$, d'où :

$$
\mathcal{F}_{T, k}(\alpha)=(1-\varphi)^{-1}\left(1-\gamma_{1}\right) \mathcal{E}_{T, k}(\alpha) \in\left(\boldsymbol{A}_{K_{1}}^{+} \otimes_{\boldsymbol{A}_{K}^{+}} \boldsymbol{N}(T)(k)\right)^{\psi_{1}=1},
$$

et la formule $\alpha \mapsto \varphi\left(\mathcal{F}_{T, k}(\alpha)\right)$ définit un homomorphisme

$$
\mathscr{D}(T)(k) \rightarrow\left(\varphi^{*} N(T)(k)\right)^{\psi=1}
$$

qui induit un diagramme commutatif :

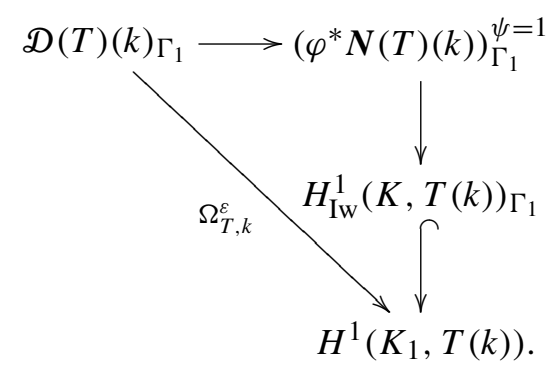

Le (3) de la proposition 3.3 entraîne l'injectivité de $\Omega_{T, k}^{\varepsilon}$ pour $k \notin[1, h]$ et l'application $\mathscr{D}(T)(k)_{\Gamma_{1}} \rightarrow\left(\varphi^{*} N(T)(k)\right)_{\Gamma_{1}}^{\psi=1}$ est donc injective. D' autre part, $\mathscr{D}(T)(k)$ et $\left(\varphi^{*} \boldsymbol{N}(T)(k)\right)^{\psi=1}$ sont des $\Lambda$-modules libres de même rang, donc $\mathscr{D}(T)(k)_{\Gamma_{1}}$ et $\left(\varphi^{*} \boldsymbol{N}(T)(k)\right)_{\Gamma_{1}}^{\psi=1}$ sont des $\mathbb{Z}_{p}$-modules libres de même rang et $\mathscr{D}(T)(k)_{\Gamma_{1}}$ est un réseau de $\left(\varphi^{*} N(T)(k)\right)_{\Gamma_{1}}^{\psi=1}$. On en déduit que l'application $\left(\varphi^{*} N(T)(k)\right)_{\Gamma_{1}}^{\psi=1} \rightarrow$ $H_{\mathrm{Iw}}^{1}(K, T(k))_{\Gamma_{1}}$ est injective et le lemme est démontré.

Remarque 3.7. On donnera plus bas une autre preuve de l'injectivité de l'application $\left(\varphi^{*} N(T)(k)\right)_{\Gamma_{1}}^{\psi=1} \rightarrow H_{\mathrm{Iw}}^{1}(K, T(k))_{\Gamma_{1}}$ (voir Proposition 4.20).

Lemme 3.8. (1) Si $f\left(X_{1}\right), g\left(X_{1}\right) \in A_{K_{1}}^{+}$, alors pour tout $k \in \mathbb{Z}$ et $n \geqslant 0$, on $a$ :

$$
\operatorname{res}\left(E_{k, 1}(f) t^{n} g\left(X_{1}\right) \frac{d X_{1}}{1+X_{1}}\right) \equiv 0 \bmod \left(p^{n} \frac{\Gamma^{*}(k)}{\Gamma^{*}(k-n)}\right) .
$$

(2) Si $m \otimes f \in \mathscr{D}(T)$ et $\beta \in\left(\varphi^{*} \boldsymbol{N}(T(-h))\right)^{\psi=1}(h-k-1)$, alors :

$$
\operatorname{res}\left(E_{k, 1}(f)\left[m, \varphi^{-1}(\beta)\right]_{V(k)} \frac{d X_{1}}{1+X_{1}}\right) \equiv 0 \bmod \left(p^{h} \frac{\Gamma^{*}(k)}{\Gamma^{*}(k-h)}\right),
$$

où $h$ est le dernier saut de la filtration de Hodge de $\boldsymbol{D}_{\mathrm{dR}}(V)$ et où on a $\Gamma^{*}(k) / \Gamma^{*}(k-n)=(k-1) \times \cdots \times(k-n)$, même si $k \leqslant 0$. 
Preuve. On commence par montrer le (1). Si $\partial_{1}=\left(1+X_{1}\right) d / d X_{1}$, alors $\partial_{1}=p \partial$ et on a :

$$
\partial_{1}\left(\frac{h\left(X_{1}\right)}{t^{n}}\right)=\frac{\partial_{1} h\left(X_{1}\right)}{t^{n}}-n p \frac{h\left(X_{1}\right)}{t^{n+1}} .
$$

On en déduit que :

$$
\operatorname{res}\left(\frac{h\left(X_{1}\right)}{t^{n+1}} \frac{d X_{1}}{1+X_{1}}\right)=\frac{1}{n p} \operatorname{res}\left(\frac{\partial_{1} h\left(X_{1}\right)}{t^{n}} \frac{d X_{1}}{1+X_{1}}\right)
$$

et par récurrence on obtient la formule :

$$
\operatorname{res}\left(\frac{h\left(X_{1}\right)}{t^{n}} \frac{d X_{1}}{1+X_{1}}\right)=\frac{1}{(n-1) ! p^{n-1}} \operatorname{res}\left(\frac{\partial_{1}^{n-1} h\left(X_{1}\right)}{X} \frac{d X_{1}}{1+X_{1}}\right) .
$$

On applique cette formule au calcul du résidu :

$$
\operatorname{res}\left(E_{k, 1}(f)\left[m, \varphi^{-1}(\beta)\right]_{V(k)} \frac{d X_{1}}{1+X_{1}}\right) .
$$

La série $E_{k, 1}$ est définie par :

$$
E_{k, 1}(f)=\sum_{j=1}^{\infty} \frac{(1-k)(2-k) \ldots(j-k-1)}{t^{j}} p^{j-1} \partial^{-j}\left(f\left(X_{1}\right)\right) .
$$

Si $n \geqslant j$, on a évidemment :

$$
\begin{aligned}
& \operatorname{res}\left(\frac{(1-k)(2-k) \ldots(j-k-1)}{t^{j}} p^{j-1} \partial^{-j} f\left(X_{1}\right) t^{n} g\left(X_{1}\right) \frac{d X_{1}}{1+X_{1}}\right) \\
& =p^{j-1}(1-k)(2-k) \ldots(j-k-1) \operatorname{res}\left(t^{n-j} \partial^{-j} f\left(X_{1}\right) g\left(X_{1}\right) \frac{d X_{1}}{1+X_{1}}\right)=0 .
\end{aligned}
$$

Si $n \leqslant j-1$, on a $\frac{(n+1-k) \times \cdots \times(j-1-k)}{(j-n-1) !} \in \mathbb{Z}$, d'où :

$$
\begin{aligned}
& \operatorname{res}\left(\frac{(1-k)(2-k) \ldots(j-k-1)}{t^{j}} p^{j-1} \partial^{-j} f\left(X_{1}\right) t^{n} g\left(X_{1}\right) \frac{d X_{1}}{1+X_{1}}\right) \\
& =\frac{(1-k)(2-k) \ldots(j-k-1) p^{j-1}}{(j-n-1) ! p^{j-n-1}} \operatorname{res}\left(\frac{\partial^{j-n-1}\left(\partial^{-j} f\left(X_{1}\right) g\left(X_{1}\right)\right)}{X} \frac{d X_{1}}{1+X_{1}}\right),
\end{aligned}
$$

et on en déduit la congruence (1) du lemme.

Montrons maintenant le (2); rappelons qu'on a posé $c_{j}=(\chi(\gamma)-1) \ldots$ $\left(\chi(\gamma)^{j}-1\right)$, où $\gamma$ est un générateur topologique de $\Gamma$. On a $X=\exp (t)-1=$ $t+t^{2} / 2 !+\cdots$, d'où :

$$
X^{j+h}=t^{j+h} \sum_{s_{1}, \ldots, s_{j+h} \geqslant 0} \frac{t^{s_{1}+\cdots+s_{j+h}}}{\left(s_{1}+1\right) ! \ldots\left(s_{j+h}+1\right) !} .
$$


Comme $m \in M$ et $\varphi^{-1}(\beta) \in A_{K_{1}}^{+} \otimes_{A_{K}^{+}} \boldsymbol{N}\left(T^{*}(-h)\right)$, on a :

$$
\left[m, \varphi^{-1}(\beta)\right]_{V}=X^{h} \sum_{j=0}^{\infty} \frac{b_{j}\left(X_{1}\right) X^{j}}{c_{j}},
$$

pour des éléments $b_{j}\left(X_{1}\right) \in \boldsymbol{A}_{K_{1}}^{+}$. Comme $p \neq 2$, en utilisant la congruence (1) du lemme et les congruences évidentes $p^{s} /(s+1) ! \equiv 0 \bmod p$ et $p^{s} / c_{s} \equiv 0 \bmod p$ pour tout $s \geqslant 1$, on montre que :

$\operatorname{res}\left(E_{k, 1}(f) b_{j}\left(X_{1}\right) \frac{t^{s_{1}+\ldots+s_{j+h}}}{\left(s_{1}+1\right) ! \ldots\left(s_{j+h}+1\right) ! c_{j}} \frac{d X_{1}}{1+X_{1}}\right) \equiv 0 \bmod \left(p^{h} \frac{\Gamma^{*}(k)}{\Gamma^{*}(k-h)}\right)$,

et la congruence (2) découle maintenant des formules (3.3) et (3.4).

Proposition 3.9. Soient $(\cdot, \cdot)_{T(k)}: H^{1}\left(K_{1}, T(k)\right) \times H^{1}\left(K_{1}, T^{*}(1-k)\right) \rightarrow \mathbb{Z}_{p}$ l'accouplement fourni par la dualité locale, $\alpha \in \mathscr{D}(T)(k), \beta \in\left(\varphi^{*} N\left(T^{*}(-h)\right)\right)^{\psi=1}$ $(h-k+1)$ et $\operatorname{cl}(\beta) \in H^{1}\left(K_{1}, T^{*}(1-k)\right)$ la classe de cohomologie associée à $\beta$ via l'injection $\left(\varphi^{*} N\left(T^{*}(-h)\right)\right)^{\psi=1}(h-k+1) \hookrightarrow H_{\mathrm{Iw}}^{1}\left(K, T^{*}(1-k)\right)$ suivie de la projection. On a alors :

$$
\left(\Omega_{T, k}^{\varepsilon}(\alpha), \operatorname{cl}(\beta)\right)_{T(k)} \equiv 0 \quad \bmod \left(p^{h} \frac{\Gamma^{*}(k)}{\Gamma^{*}(k-h)}\right) .
$$

Preuve. Soit $A \in \varphi^{-1}\left(\boldsymbol{D}\left(T^{*}(1-k)\right)^{\psi=0}\right)$ une solution de l'équation $\left(\gamma_{1}-1\right) A=$ $\varphi^{-1}(\varphi-1) \beta$. La formule du cup-produit en termes de $(\varphi, \Gamma)$-modules (voir [Her01, proposition 4.4]) s'écrit :

$$
\left(\Omega_{T, k}^{\varepsilon}(\alpha), \operatorname{cl}(\beta)\right)_{T(k)}=-\operatorname{cl}\left(\left[\gamma_{1} \mathcal{E}_{T, k}(\alpha), \varphi^{-1}(\beta)\right]_{V(k)}-\left[\varphi \mathcal{F}_{T, k}(\alpha), A\right]_{V(k)}\right) .
$$

Pour calculer cette classe, on reprend les arguments de la preuve du théorème 5.1.2 de [Ben00]. En termes de $(\varphi, \Gamma)$-modules, l'isomorphisme canonique $H^{2}\left(K_{n}, \mathbb{Z}_{p}(1)\right) \simeq$ $\mathbb{Z}_{p}$ est donné par la formule (voir [Ben00, théorème 2.2.6]) :

$$
\begin{aligned}
\operatorname{TR}_{n}: H^{2}\left(\varphi^{-n}\left(C_{\varphi, \gamma_{n}}\left(K_{n}, \mathbb{Z}_{p}(1)\right)\right)\right) & \stackrel{\sim}{\rightarrow} \mathbb{Z}_{p} \\
\left(\operatorname{cl}\left(h\left(X_{n}\right) \otimes \varepsilon\right)\right) & \mapsto-\frac{p^{n}}{\log \chi\left(\gamma_{n}\right)} \operatorname{Tr}_{K / \mathbb{Q}_{p}}\left(\operatorname{res} \frac{h\left(X_{n}\right) d X_{n}}{1+X_{n}}\right) .
\end{aligned}
$$

Si $\alpha=f \otimes m \in \mathcal{O}_{K}[[X]]^{\psi=0} \otimes_{\mathcal{O}_{K}} M$, alors il existe $y$ tel que

$$
\mathcal{E}_{T, k}(\alpha)=E_{k, 1}(f) \otimes m+(\varphi-1) y, \quad \mathcal{F}_{T, k}(\alpha)=F_{T, k}(\alpha)+\left(1-\gamma_{1}\right) y,
$$

et

$$
(\varphi-1) F_{T, k}(\alpha)=\left(\gamma_{1}-1\right)\left(E_{k, 1}(f) \otimes m\right) .
$$


On en déduit que :

$\left(\Omega_{T, k}^{\varepsilon}(\alpha), \operatorname{cl}(\beta)\right)_{T(k)}$

$=\frac{p}{\log \chi\left(\gamma_{1}\right)} \operatorname{Tr}_{K / \mathbb{Q}_{p}}\left(\operatorname{res}\left(\left[\gamma_{1} E_{T, k}(f) \otimes m, \varphi^{-1}(\beta)\right]_{V(k)}-\left[\varphi F_{T, k}(\alpha), A\right]_{V(k)}\right) \frac{d X_{1}}{1+X_{1}}\right)$.

Comme $\psi_{1}(A)=0$, on a $\psi_{1}\left(\left[\varphi F_{T, k}(\alpha), A\right]_{V(k)}\right)=0$, d'où (cf. [Ben00, lemme 2.2.2.1]) :

$$
\operatorname{res}\left(\left[\varphi F_{T, k}(\alpha), A\right]_{V(k)} \frac{d X_{1}}{1+X_{1}}\right)=0 .
$$

D'autre part, comme $\left(\gamma_{1}-1\right) E_{k, 1}(f) \in \mathbb{Q}_{p}\left[\left[X_{1}\right]\right]$ le (2) du lemme 3.8 nous donne :

$$
\begin{aligned}
& \operatorname{res}\left(\left[\gamma_{1} E_{T, k}(f) \otimes m, \varphi^{-1}(\beta)\right]_{V(k)} \frac{d X_{1}}{1+X_{1}}\right) \\
& \quad=\operatorname{res}\left(\left[E_{T, k}(f) \otimes m, \varphi^{-1}(\beta)\right]_{V(k)} \frac{d X_{1}}{1+X_{1}}\right) \equiv 0 \bmod \left(p^{h} \frac{\Gamma^{*}(k)}{\Gamma^{*}(k-h)}\right) .
\end{aligned}
$$

Preuve de la proposition 3.5. La (3) de la proposition 3.4 nous donne :

$$
\begin{aligned}
\operatorname{det}_{\mathbb{Z}_{p}}\left(\Omega_{T, k}^{\varepsilon}\left(\mathscr{D}(T)(k)_{\Gamma_{1}}\right), \Omega_{T^{*}(-h), 1+h-k}^{\varepsilon}\left(\mathscr{D}\left(T^{*}(-h)\right)(1+h-k)_{\Gamma_{1}}\right)\right)_{T(k)} \\
=\left(p^{h} \frac{\Gamma^{*}(k)}{\Gamma^{*}(k-h)}\right)^{\left[K_{1}: \mathbb{Q}_{p}\right] d} \mathbb{Z}_{p},
\end{aligned}
$$

où $d=\operatorname{dim}(V)$. Par ailleurs, la proposition 3.9 donne l'inclusion :

$$
\begin{aligned}
\operatorname{det}_{\mathbb{Z}_{p}}\left(\left(\varphi^{*} N(T)(k)\right)_{\Gamma_{1}}^{\psi=1}, \Omega_{T^{*}(-h), 1+h-k}^{\varepsilon}\left(D\left(T^{*}(-h)\right)(1+h-k)_{\Gamma_{1}}\right)\right)_{T(k)} & \\
& \subset\left(p^{h} \frac{\Gamma^{*}(k)}{\Gamma^{*}(k-h)}\right)^{\left[K_{1}: \mathbb{Q}_{p}\right] d} \mathbb{Z}_{p} .
\end{aligned}
$$

Comme l'inclusion $\mathscr{D}(T)(k)_{\Gamma_{1}} \hookrightarrow\left(\varphi^{*} N(T)(k)\right)_{\Gamma_{1}}^{\psi=1}$ est déjà établie, on en déduit que $\mathscr{D}(T)(k)_{\Gamma_{1}} \stackrel{\sim}{\rightarrow}\left(\varphi^{*} N(T)(k)\right)_{\Gamma_{1}}^{\psi=1}$ et la proposition 3.5 est démontrée.

Corollaire 3.10. Si $k \notin[1, h]$, alors :

$$
\begin{gathered}
{\left[\boldsymbol{D}(T(k))_{\Gamma_{1}}^{\psi=1}:\left(\varphi^{*} \boldsymbol{N}(T)(k)\right)_{\Gamma_{1}}^{\psi=1}\right]} \\
{\left[\boldsymbol{D}\left(T^{*}(1-k)\right)_{\Gamma_{1}}^{\psi=1}:\left(\varphi^{*} \boldsymbol{N}\left(T^{*}(-h)\right)(h+1-k)\right)_{\Gamma_{1}}^{\psi=1}\right]} \\
=\left(p^{h} \frac{\Gamma^{*}(k)}{\Gamma^{*}(k-h)}\right)^{\left[K_{1}: \mathbb{Q}_{p}\right] \operatorname{dim}(V)} .
\end{gathered}
$$


Preuve. Soit $\tilde{H}^{1}\left(K_{1}, T(k)\right)=H^{1}\left(K_{1}, T(k)\right) / H^{1}\left(K_{1}, T(k)\right)_{\text {tor. Comme par hypo- }}$ thèse $V(k)^{G_{K}}=0$, on a $H^{1}\left(K_{1}, T(k)\right)_{\text {tor }} \simeq H^{0}\left(K_{1}, V(k) / T(k)\right)$. Soit

$$
0 \rightarrow H_{\mathrm{IW}}^{1}(K, T(k))_{\Gamma_{1}} \rightarrow H^{1}(K, T(k)) \rightarrow H_{\mathrm{IW}}^{2}(K, T(k))^{\Gamma_{1}} \rightarrow 0
$$

la suite exacte de la proposition 2.8. Comme $V$ n'a pas de sous-quotient isomorphe à $\mathbb{Q}_{p}(m)$, on a $V^{*}(1-k)^{H_{K}}=0$ et donc $H_{\mathrm{IW}}^{2}(K, T(k))$ est fini et on a

$$
\sharp H_{\mathrm{IW}}^{2}(K, T(k))^{\Gamma_{1}}=\sharp H^{0}\left(K_{1}, V^{*}(1-k) / T^{*}(1-k)\right)
$$

(voir le (4) de la remarque 2.9). On en déduit :

$$
\begin{aligned}
& {\left[H_{\mathrm{IW}}^{1}(K, T(k))_{\Gamma_{1}}:\left(\varphi^{*} N(T)(k)\right)_{\Gamma_{1}}^{\psi=1}\right]} \\
& \quad=\frac{\sharp H^{0}\left(K_{1}, V(k) / T(k)\right)}{\sharp H^{0}\left(K_{1}, V^{*}(1-k) / T^{*}(1-k)\right)}\left[\tilde{H}^{1}\left(K_{1}, T(k)\right):\left(\varphi^{*} N(T)(k)\right)_{\Gamma_{1}}^{\psi=1}\right],
\end{aligned}
$$

d'où :

$$
\begin{aligned}
& {\left[H_{\mathrm{Iw}}^{1}(K, T(k))_{\Gamma_{1}}:\left(\varphi^{*} N(T)(k)\right)_{\Gamma_{1}}^{\psi=1}\right]} \\
& {\left[H_{\mathrm{IW}}^{1}\left(K, T^{*}(1-k)\right)_{\Gamma_{1}}:\left(\varphi^{*} N\left(T^{*}(-h)\right)(h+1-k)\right)_{\Gamma_{1}}^{\psi=1}\right]} \\
& =\left[\tilde{H}^{1}\left(K_{1}, T(k)\right):\left(\varphi^{*} N(T)(k)\right)_{\Gamma_{1}}^{\psi=1}\right] \\
& {\left[\tilde{H}^{1}\left(K_{1}, T^{*}(1-k)\right):\left(\varphi^{*} N\left(T^{*}(-h)\right)(h+1-k)\right)_{\Gamma_{1}}^{\psi=1}\right] .}
\end{aligned}
$$

Par la proposition 3.5, ce produit est égal au déterminant :

$$
\operatorname{det}_{\mathbb{Z}_{p}}\left(\Omega_{T, k}^{\varepsilon}\left(\mathscr{D}(T)(k)_{\Gamma_{1}}\right), \Omega_{T^{*}(-h), 1+h-k}^{\varepsilon}\left(\mathscr{D}\left(T^{*}(-h)\right)(1+h-k)_{\Gamma_{1}}\right)\right)_{T(k)}
$$

qui est égal à $\left(p^{h} \Gamma^{*}(k) / \Gamma^{*}(k-h)\right)^{\left[K_{1}: \mathbb{Q}_{p}\right] \operatorname{dim}(V)}$. Le corollaire résulte alors du fait que $\boldsymbol{D}(T(k))^{\psi=1} \simeq H_{\mathrm{IW}}^{1}(K, T(k))$.

\section{La conjecture $C_{\mathrm{Iw}}\left(K_{\infty} / K, V\right)$}

Dans ce chapitre, on énonce la conjecture $C_{\mathrm{Iw}}\left(K_{\infty} / K, V\right)$ puis on montre qu'elle est équivalente $C_{\mathrm{EP}}\left(K_{n} / K, V\right)$ pour tout $n \geqslant 1$ et finalement, on démontre la conjecture $C_{\mathrm{Iw}}\left(K_{\infty} / K, V\right)$.

4.1. Enoncé de la conjecture. Dans ce paragraphe, on énonce la conjecture $C_{\text {Iw }}\left(K_{\infty} / K, V\right)$ (c'est la conjecture que Perrin-Riou appelle $\delta_{\mathbb{Z}_{p}}(V)$ ). On commence par des rappels et des compléments sur la loi de réciprocité explicite. 
Soit $(\cdot, \cdot)_{T, n}: H^{1}\left(K_{n}, T\right) \times H^{1}\left(K_{n}, T^{*}(1)\right) \rightarrow \mathbb{Z}_{p}$ l'accouplement fourni par la dualité locale. On définit une application bilinéaire :

$$
\langle\cdot, \cdot\rangle_{T}: H_{\mathrm{Iw}}^{1}(K, T) \times H_{\mathrm{Iw}}^{1}\left(K, T^{*}(1)\right)^{\iota} \rightarrow \Lambda,
$$

en imposant que pour tout $n \geqslant 1$, on ait :

$$
\langle x, y\rangle_{T} \equiv \sum_{\tau \in G_{n}}\left(\tau^{-1} x_{n}, y_{n}\right)_{T, n} \tau \bmod \left(\gamma_{n}-1\right) .
$$

Par linéarité, on obtient un accouplement :

$$
\langle\cdot, \cdot\rangle_{V}: \mathcal{K}(\Gamma) \otimes_{\Lambda} H_{\mathrm{Iw}}^{1}(K, T) \times \mathcal{K}(\Gamma) \otimes_{\Lambda} H_{\mathrm{Iw}}^{1}\left(K, T^{*}(1)\right)^{\iota} \rightarrow \mathcal{K}(\Gamma) .
$$

D'autre part, en posant $(1+X) \star(1+X)=1+X$, on étend la dualité canonique $\boldsymbol{D}_{\mathrm{dR}}^{K}(V) \times \boldsymbol{D}_{\mathrm{dR}}^{K}\left(V^{*}(1)\right) \rightarrow K$ en une forme $\Lambda$-bilinéaire :

$$
\star_{\mathscr{D}(V)}: \mathscr{D}(V) \times \mathscr{D}\left(V^{*}(1)\right) \rightarrow K \otimes_{\mathcal{O}_{K}} \mathcal{O}_{K}[[X]]^{\psi=0} .
$$

Le théorème suivant est la loi de réciprocité de Perrin-Riou (la conjecture $\operatorname{Rec}(V)$ de [Per94]).

Théorème 4.1. Si V est une représentation cristalline de $G_{K}$, alors pour tout h on a :

$$
\left\langle\operatorname{Exp}_{V, h}^{\varepsilon}(f), \operatorname{Exp}_{V^{*}(1), 1-h}^{\varepsilon^{-1}}\left(g^{l}\right)\right\rangle_{V}(1+X)=(-1)^{h-1} \operatorname{Tr}_{K / \mathbb{Q}_{p}}(f \star \mathscr{D}(V) g) .
$$

On dispose de plusieurs démonstrations de ce résultat : voir [Col98], [KKT96], [Ben00], [Ber03]. On note $\Lambda_{\mathbb{Q}_{p}}$ l'anneau $\mathbb{Q}_{p} \otimes_{\mathbb{Z}_{p}} \Lambda$, et on pose :

$$
\begin{aligned}
\Delta_{\mathrm{PR}}\left(K_{\infty} / K, V\right) & =\operatorname{det}_{\Lambda_{\mathbb{Q} p}} \mathbb{R} \Gamma_{\mathrm{IW}}(K, V) \otimes \operatorname{det}_{\Lambda_{\mathbb{Q} p}} \mathscr{D}(V) \\
& \simeq \otimes_{i=1}^{2}\left(\operatorname{det}_{\Lambda_{\mathbb{Q}_{p}}} H_{\mathrm{Iw}}^{i}(K, V)\right)^{(-1)^{i}} \otimes \operatorname{det}_{\Lambda_{\mathbb{Q}_{p}}} \mathscr{D}(V) .
\end{aligned}
$$

Comme $H_{\mathrm{Iw}}^{1}(K, T) / T^{H_{K}}$ est un $\Lambda$-module sans torsion, de $\operatorname{rang}\left[K: \mathbb{Q}_{p}\right] \operatorname{dim}(V)$ (voir le (5) de la remarque 2.9), $H_{\mathrm{Iw}}^{1}(K, V) / V^{H_{K}}$ est un $\Lambda_{\mathbb{Q}_{p}}$-module libre du même rang. Comme $H_{\mathrm{IW}}^{2}(K, V)$ est un $\Lambda_{\mathbb{Q}_{p}}$-module de type fini de torsion, le déterminant de l'application exponentielle élargie induit une application :

$$
\delta_{V, K_{\infty} / K, h}^{\prime}: \Delta_{\mathrm{PR}}\left(K_{\infty} / K, V\right) \rightarrow \mathscr{H}(\Gamma) .
$$

Soient $\boldsymbol{\Gamma}_{h}(V)=\prod_{j>-h}\left(\ell_{-j}\right)^{\operatorname{dim}_{\mathbb{Q}_{p}} \operatorname{Fil}^{j} \boldsymbol{D}_{\text {cris }}(V)}$ et $\delta_{V, K_{\infty} / K}^{\prime}=\boldsymbol{\Gamma}_{h}(V)^{-1} \delta_{V, K_{\infty} / K, h}^{\prime}$. Un petit calcul montre que l'application $\delta_{V, K_{\infty} / K}^{\prime}: \Delta_{\mathrm{PR}}\left(K_{\infty} / K, V\right) \rightarrow \mathcal{K}(\Gamma)$ ne dépend pas de $h$, et le théorème 4.1 entraîne le résultat suivant (c'est l'ancienne conjecture $\delta_{\mathbb{Q}_{p}}(V)$ de [Per94]). 
Théorème 4.2. On a $\delta_{V, K_{\infty} / K}^{\prime}\left(\Delta_{\mathrm{PR}}\left(K_{\infty} / K, V\right)\right)=\Lambda_{\mathbb{Q}_{p}}$.

Preuve. Voir le théorème 3.4.2 et la proposition 3.6.6 de [Per94].

Nous allons maintenant donner une version entière de la conjecture $\delta_{\mathbb{Q}_{p}}(V)$ cidessus, c'est la conjecture $\delta_{\mathbb{Z}_{p}}(V)$ de Perrin-Riou. Soient :

$$
\begin{aligned}
\Delta_{\mathrm{Iw}}\left(K_{\infty} / K, T\right) & =\operatorname{det}_{\Lambda} \mathbb{R} \Gamma_{\mathrm{Iw}}(K, T) \otimes_{\Lambda} \operatorname{det}_{\Lambda}\left(\operatorname{Ind}_{K_{\infty} / \mathbb{Q}_{p}} T\right) \\
& \simeq \otimes_{i=1}^{2}\left(\operatorname{det}_{\Lambda} H_{\mathrm{Iw}}^{i}(K, T)\right)^{(-1)^{i}} \otimes_{\Lambda} \operatorname{det}_{\Lambda}\left(\operatorname{Ind}_{K_{\infty} / \mathbb{Q}_{p}} T\right),
\end{aligned}
$$

et $\Delta_{\text {Iw }}\left(K_{\infty} / K, V\right)=\Delta_{\text {Iw }}\left(K_{\infty} / K, T\right) \otimes_{\mathbb{Z}_{p}} \mathbb{Q}_{p}$.

Soit $a_{V, K} \in \mathbb{Z}_{p}^{\times}$l'élément défini au paragraphe 2.4 et soit :

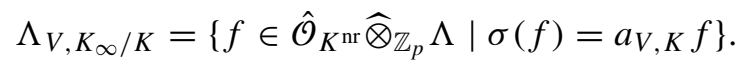

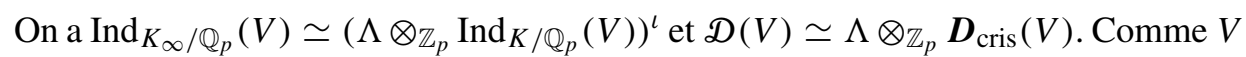
est cristalline, on a $\boldsymbol{D}_{\text {pst }}(V)=K^{\mathrm{nr}} \otimes_{K} \boldsymbol{D}_{\text {cris }}(V)$ et le lemme 2.16 donne $\varepsilon(K, V)=1$. L'application $\alpha_{V, K}$ induit donc par linéarité un homomorphisme :

$$
\alpha_{V, K_{\infty} / K}: \operatorname{det}_{\Lambda_{\mathbb{Q} p}}^{-1} \mathcal{D}(V) \otimes \operatorname{det}_{\Lambda_{\mathbb{Q}_{p}}}\left(\operatorname{Ind}_{K_{\infty} / \mathbb{Q}_{p}}(V)\right) \rightarrow \Lambda_{V, K_{\infty} / K} \otimes_{\mathbb{Z}_{p}} \mathbb{Q}_{p}
$$

En le composant avec $\delta_{V, K_{\infty} / K}^{\prime}$, on obtient une trivialisation canonique :

$$
\delta_{V, K_{\infty} / K}: \Delta_{\mathrm{Iw}}\left(K_{\infty} / K, V\right) \stackrel{\sim}{\rightarrow} \Lambda_{V, K_{\infty} / K} \otimes_{\mathbb{Z}_{p}} \mathbb{Q}_{p} .
$$

Conjecture 4.3 $\left(C_{\mathrm{Iw}}\left(K_{\infty} / K, V\right)\right)$. On a $\delta_{V, K_{\infty} / K}\left(\Delta_{\mathrm{Iw}}\left(K_{\infty} / K, T\right)\right)=\Lambda_{V, K_{\infty} / K}$.

Cette conjecture est démontrée dans le paragraphe 4.4, c'est le théorème 4.22.

4.2. Équivalence de $\boldsymbol{C}_{\mathrm{Iw}}$ et de $\boldsymbol{C}_{\mathrm{EP}}$ : étude de $\boldsymbol{\Xi}_{\boldsymbol{V}, \boldsymbol{n}}^{\boldsymbol{\varepsilon}}$. Ce paragraphe et le suivant sont consacrés à la démonstration du théorème suivant.

Théorème 4.4. Pour tout $n \geqslant 1$, la conjecture $C_{\mathrm{Iw}}\left(K_{\infty} / K, V\right)$ est équivalente à la conjecture $C_{\mathrm{EP}}\left(K_{n} / K, V\right)$.

Afin de montrer le théorème ci-dessus, nous avons besoin de résultats de descente. La technique générale de descente des complexes a été développée par Nekovàr (voir [Nek02, §11.6] ainsi que [BG03, lemme 8.1]). Nous avons besoin d'un cas très particulier de cette théorie, qui est sans doute bien connu, et qui en tout cas se démontre facilement.

Dans cette section, on pose $\mathscr{H}=\mathscr{H}(\Gamma)$ pour alléger la notation. Si $M$ et $N$ sont deux $\mathscr{H}$-modules libres de même rang et si $f: M \rightarrow N$ est un homomorphisme injectif, on note :

$$
\operatorname{det}(f): \operatorname{det}_{\mathscr{H}} M \otimes \operatorname{det}_{\mathscr{H}}^{-1} N \rightarrow \mathscr{H}
$$


l'homomorphisme qui s'en déduit. Pour tout $n \geqslant 0$, on a une projection naturelle $\mathscr{H} \rightarrow \mathbb{Q}_{p}\left[G_{n}\right]$. L'algèbre $\mathbb{Q}_{p}\left[G_{n}\right]$ se décompose en produit de corps $\mathbb{Q}_{p}\left[G_{n}\right] \simeq$ $\bigoplus_{\lambda} E_{\lambda}$, et on note $\mathfrak{p}(\lambda)$ le noyau de la projection $\mathcal{H} \rightarrow E_{\lambda}$. On pose $M_{\lambda}=E_{\lambda} \otimes \mathscr{H} M$, et on note $f_{\lambda}: M_{\lambda} \rightarrow N_{\lambda}$ l'homomorphisme de $E_{\lambda}$-modules qui se déduit de $f$. Le diagramme commutatif :

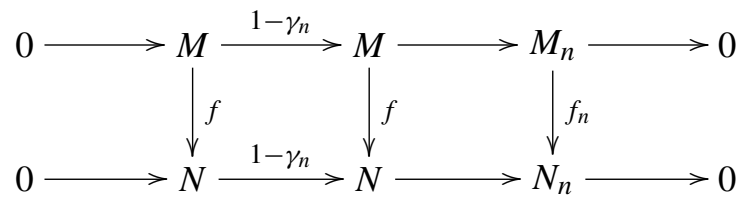

donne lieu à des isomorphismes canoniques :

$$
\begin{aligned}
\operatorname{ker}\left(f_{n}\right) & \simeq \operatorname{coker}(f)^{\Gamma_{n}}, \\
\operatorname{coker}\left(f_{n}\right) & \simeq \operatorname{coker}(f)_{\Gamma_{n}} .
\end{aligned}
$$

On dit que $f$ est $\lambda$-semi-simple si la $\lambda$-composante $B_{f, \lambda}$ de l'application :

$$
B_{f, n}: \operatorname{ker}\left(f_{n}\right) \hookrightarrow \operatorname{coker}(f) \rightarrow \operatorname{coker}\left(f_{n}\right)
$$

est un isomorphisme. Dans ce cas on a un isomorphisme canonique :

$$
i_{f, \lambda}: \operatorname{det}_{E_{\lambda}} M_{\lambda} \otimes \operatorname{det}_{E_{\lambda}}^{-1} N_{\lambda} \simeq \operatorname{det}_{E_{\lambda}} \operatorname{ker}\left(f_{\lambda}\right) \otimes \operatorname{det}_{E_{\lambda}}^{-1} \operatorname{coker}\left(f_{\lambda}\right) \simeq E_{\lambda},
$$

le deuxieme isomorphisme étant induit par $B_{f, \lambda}$.

On dit que $f$ est $\lambda$-admissible si l'image $\operatorname{de} \operatorname{det}(f)$ s'écrit sous la forme $\left(1-\gamma_{n}\right)^{r_{\lambda}} h \mathscr{H}$, où $h$ est une unité de $\mathscr{H}_{\mathfrak{p}(\lambda)}$ et $r_{\lambda}=\operatorname{dim}_{E_{\lambda}}\left(\right.$ ker $\left.f_{\lambda}\right)$. On dit que $f$ est admissible si elle est $\lambda$-admissible pour tout $\lambda$ et on pose $\operatorname{det}^{*}(f)_{\lambda}=\left(1-\gamma_{n}\right)^{-r_{\lambda}} \operatorname{det}(f)$.

Lemme 4.5. On conserve les hypothèses concernant $f: M \rightarrow N$. Si f est $\lambda$-admissible, alors $f$ est $\lambda$-semi-simple et le diagramme suivant est commutatif:

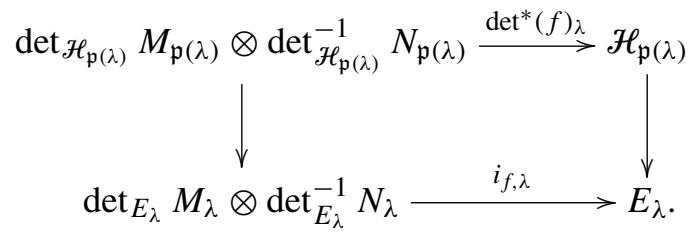

Preuve. Soit $X_{1}, \ldots, X_{r_{\lambda}} \in M_{\mathfrak{p}(\lambda)}$ un relèvement d'une base $x_{1}, \ldots x_{r_{\lambda}}$ de ker $f_{\lambda}$. Comme $M_{\lambda}$ est un facteur direct de $M_{n}$ on peut choisir $X_{i}$ de telle façon que $f\left(X_{i}\right)=$ $\left(1-\gamma_{n}\right) A_{i}$ où $A_{i} \in N_{\mathfrak{p}(\lambda)}$. On fixe un complément $X_{r_{\lambda}+1}, \ldots, X_{m} \operatorname{de} X_{1}, \ldots, X_{r_{\lambda}}$ à une base de $M_{\mathfrak{p}(\lambda)}$. Soit $Y_{1}, \ldots, Y_{r_{\lambda}} \in N_{\mathfrak{p}(\lambda)}$ un relèvement d'une base $y_{1}, \ldots, y_{r_{\lambda}}$ de 
$\operatorname{coker}\left(f_{\lambda}\right)$. Les éléments $Y_{1}, \ldots, Y_{r_{\lambda}}, Y_{r_{\lambda}+1}=f\left(X_{r_{\lambda}+1}\right), \ldots, Y_{m}=f\left(X_{m}\right)$ forment alors une base de $N_{\mathfrak{p}(\lambda)}$ et on a :

$$
\begin{aligned}
\operatorname{det}(f) & \left(\wedge_{i=1}^{m} X_{i} \otimes \wedge_{j=1}^{m} Y_{j}^{*}\right) \\
= & \operatorname{det}\left(\wedge_{i=1}^{r_{\lambda}} X_{i} \otimes \wedge_{j=1}^{r_{\lambda}} Y_{j}^{*}\right)=\left(1-\gamma_{n}\right)^{r_{\lambda}} \operatorname{det}_{1 \leqslant i, j \leqslant r_{\lambda}}\left(Y_{j}^{*}\left(A_{i}\right)\right),
\end{aligned}
$$

ce qui montre le lemme.

En particulier, soit $N$ un $\Lambda_{\mathbb{Q}_{p}}$-module de torsion et de type fini; il admet une résolution projective $0 \rightarrow P_{1} \stackrel{f}{\rightarrow} P_{0} \rightarrow N \rightarrow 0$, où $\operatorname{rg}\left(P_{0}\right)=\operatorname{rg}\left(P_{1}\right)$. Pour tout $\lambda$, on note $\Lambda_{\mathfrak{p}(\lambda)}$ la localisation de $\Lambda_{\mathbb{Q}_{p}}$ en $\mathfrak{p}(\lambda)$. On dit que $N$ est $\lambda$-admissible si $f: P_{1} \rightarrow P_{0}$ l'est. Dans ce cas, le lemme 4.5 fournit un diagramme commutatif :

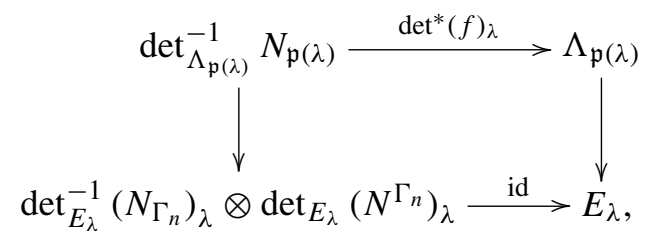

où la deuxième ligne est induite par la projection $N^{\Gamma_{n}} \rightarrow N_{\Gamma_{n}}$.

On fixe un isomorphisme $\mathbb{Z}_{p}\left[\left[\Gamma_{1}\right]\right] \simeq \mathbb{Z}_{p}[[T]]$ en envoyant $\gamma_{1}$ sur $1+T$. On pose :

$$
\delta_{i}=\frac{1}{\sharp \Delta_{K}} \sum_{g \in \Delta_{K}} \chi^{i}\left(g^{-1}\right) g,
$$

ce qui fait que $\Lambda=\bigoplus_{i=0}^{p-2} \Lambda_{i}$, où $\Lambda_{i}=\delta_{i} \mathbb{Z}_{p}\left[\left[\Gamma_{1}\right]\right]$.

Il est clair que $\delta_{i}\left(\mathbb{Z}_{p}(j)\right)=0$ si $i \neq j \bmod (p-1)$. Sinon, on a une suite exacte $0 \rightarrow \mathbb{Z}_{p}[[T]] \stackrel{f_{j}}{\rightarrow} \mathbb{Z}_{p}[[T]] \rightarrow \mathbb{Z}_{p}(j) \rightarrow 0$, où $f_{j}$ est la multiplication par $\left(\chi\left(\gamma_{1}\right)^{j}-1\right)-T$, ce qui fait que $\operatorname{det}_{\Lambda_{i}}^{-1} \mathbb{Z}_{p}(j)=\left(\left(\chi\left(\gamma_{1}\right)^{j}-1\right)-T\right) \Lambda_{i}$ si $i=j$ $\bmod (p-1)$. En particulier, $\mathbb{Q}_{p}(j)$ est admissible.

Proposition 4.6. Si $h \geqslant 1$ est un entier tel que $\mathrm{Fil}^{-h} \boldsymbol{D}_{\mathrm{dR}}^{K}(V)=\boldsymbol{D}_{\mathrm{dR}}^{K}(V)$, alors l'application $\operatorname{Exp}_{V, h}^{\varepsilon}$ est admissible.

Nous allons déduire cette proposition du théorème 4.2. Pour alléger les notations, posons $a_{j}=\operatorname{dim}_{\mathbb{Q}_{p}} \operatorname{Fil}^{j} \boldsymbol{D}_{\mathrm{dR}}^{K}(V), b_{j}=\operatorname{dim}_{\mathbb{Q}_{p}} \boldsymbol{D}_{\text {cris }}(V)^{\varphi=p^{-j}}$ et $\omega_{k}(T)=$ $(1+T)^{p^{k}}-1$. On commence par un lemme purement technique.

Lemme 4.7. Pour tout $n \geqslant 1$, on $a \boldsymbol{\Gamma}_{h}(V) \equiv \Gamma_{h}^{*}(V) \omega_{n-1}(T)^{a_{0}} \bmod \omega_{n-1}(T)^{a_{0}+1}$, où $\boldsymbol{\Gamma}_{h}^{*}(V)= \pm(h-1) !^{\operatorname{dim}_{\mathbb{Q}_{p}} \boldsymbol{D}_{\text {cris }}(V)}\left(\log \chi\left(\gamma_{n}\right)\right)^{-a_{0}} \Gamma^{*}(V)^{-1}$. 
Preuve. Comme :

on a :

$$
\frac{\log (\gamma)}{\log \chi(\gamma)}=\frac{\log \left(\gamma_{n}\right)}{\log \chi\left(\gamma_{n}\right)} \equiv \log ^{-1} \chi\left(\gamma_{n}\right) \omega_{n-1}(T) \bmod \omega_{n-1}(T)^{2},
$$

$$
\boldsymbol{\Gamma}_{h}(V) \equiv \pm\left(\log \chi\left(\gamma_{n}\right)\right)^{-a_{0}}\left(\prod_{\substack{j>-h \\ j \neq 0}} j^{a_{j}}\right) \omega_{n-1}(T)^{a_{0}} \bmod \omega_{n-1}(T)^{a_{0}+1} .
$$

Un calcul facile montre alors que :

$$
\begin{aligned}
(h-1) !^{\operatorname{dim}_{\mathbb{Q}_{p}} \boldsymbol{D}_{\text {cris }}(V)} & = \pm\left(\prod_{\substack{j>-h \\
j \neq 0}} j^{a_{j}}\right) \prod_{i \geqslant 0} \Gamma^{*}(h-i)^{\left[K: \mathbb{Q}_{p}\right] h_{i-h}(V)} \\
& = \pm\left(\prod_{\substack{j>-h \\
j \neq 0}} j^{a_{j}}\right) \Gamma^{*}(V),
\end{aligned}
$$

d'où le lemme.

Preuve de la proposition 4.6. Il résulte de la suite exacte (3.1) que l'image de :

$$
\operatorname{det}_{\Lambda_{\mathbb{Q} p}} \mathscr{D}(V)^{\Delta=0} \otimes \operatorname{det}_{\Lambda_{\mathbb{Q}_{p}}}^{-1}\left(\frac{H_{\mathrm{IW}}^{1}(K, V)}{V^{H_{K}}}\right)
$$

dans $\mathscr{H}(\Gamma)$ est égale à :

$$
v=\Gamma_{h}(V) \operatorname{det}_{\Lambda_{\mathbb{Q}_{p}}}\left(V^{H_{K}}\right) \operatorname{det}_{\Lambda_{\mathbb{Q}_{p}}}^{-1}\left(V^{*}(1)^{H_{K}}\right)^{*} \prod_{j \in \mathbb{Z}}\left(\operatorname{det}_{\Lambda_{\mathbb{Q}_{p}}}^{-1} \mathbb{Q}_{p}(j)\right)^{b_{j}} .
$$

Fixons un $\lambda$ et notons $n$ le plus petit entier tel que $E_{\lambda} \subset \mathbb{Q}_{p}\left[G_{n}\right]$.

Supposons d'abord que $\lambda \neq \lambda_{0}\left(\lambda_{0}\right.$ correspond à l'inclusion de $\mathbb{Q}_{p}$ dans $\left.\mathbb{Q}_{p}\left[G_{n}\right]\right)$.

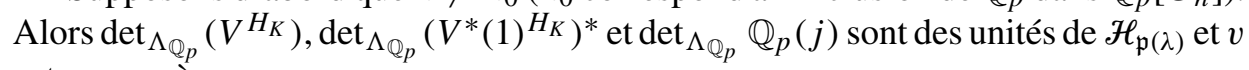
est congru à :

$$
\begin{array}{r}
\Gamma_{h}^{*}(V) \operatorname{det}_{\Lambda_{\mathbb{Q} p}}\left(V^{H_{K}}\right) \operatorname{det}_{\Lambda_{\mathbb{Q}_{p}}}^{-1}\left(V^{*}(1)^{H_{K}}\right)^{*} \prod_{j \in \mathbb{Z}} \operatorname{det}_{\Lambda_{\mathbb{Q}_{p}}} \mathbb{Q}_{p}(j)^{-b_{j}} \omega_{n-1}(T)^{a_{0}} \\
\bmod \omega_{n-1}(T)^{a_{0}+1} .
\end{array}
$$

D'autre part, la suite exacte (3.2) nous donne $\operatorname{ker}\left(\operatorname{Exp}_{V, h}^{\varepsilon}\right)_{\lambda} \simeq \operatorname{Fil}^{0} D_{\mathrm{dR}}^{K_{n}}(V)_{\lambda}$, ce qui fait que $\operatorname{dim}_{E_{\lambda}}\left(\operatorname{ker}\left(\operatorname{Exp}_{V, h}^{\varepsilon}\right) \lambda\right)=a_{0} \operatorname{et}^{\operatorname{Exp}_{V, h}^{\varepsilon}}$ est bien $\lambda$-admissible.

Supposons maintenant que $\lambda=\lambda_{0}$. Le même calcul montre alors que $v$ est congru à :

$$
\begin{array}{r}
\Gamma_{h}^{*}(V) \operatorname{det}_{\Lambda_{\mathbb{Q}_{p}}}\left(V^{H_{K}} / V^{G_{K}}\right) \operatorname{det}_{\Lambda_{\mathbb{Q}_{p}}}^{-1}\left(V^{*}(1)^{H_{K}} / V^{*}(1)^{G_{K}}\right)^{*} \prod_{j \in \mathbb{Z}} \operatorname{det}_{\Lambda_{\mathbb{Q}_{p}}} \mathbb{Q}_{p}(j)^{-b_{j}} T^{r} \\
\bmod T^{r+1},
\end{array}
$$


où :

$$
\begin{aligned}
r= & \operatorname{dim}_{\mathbb{Q}_{p}}\left(\operatorname{ker}\left(\operatorname{Exp}_{V, h, n}^{\varepsilon}\right)_{\lambda_{0}}\right) \\
= & \operatorname{dim}_{\mathbb{Q}_{p}}\left(\boldsymbol{D}_{\text {cris }}(V)^{\varphi=1}\right)+\operatorname{dim}_{\mathbb{Q}_{p}}\left(\operatorname{Fil}^{0} \boldsymbol{D}_{\text {cris }}(V)\right. \\
& -\operatorname{dim}_{\mathbb{Q}_{p}}\left(V^{G_{K}}\right)+\operatorname{dim}_{\mathbb{Q}_{p}}\left(V^{*}(1)^{G_{K}}\right),
\end{aligned}
$$

et $\operatorname{Exp}_{V, h}^{\varepsilon}$ est bien $\lambda$-admissible dans ce cas aussi.

Nous allons maintenant calculer le déterminant de l'application $\Xi_{V, n}^{\varepsilon}$; ces calculs généralisent (et corrigent ...) ceux de [Per94, lemme 3.5.7]. Si :

$$
\Delta_{0}: \mathscr{D}(V) \rightarrow \boldsymbol{D}_{\text {cris }}(V) /(1-\varphi) \boldsymbol{D}_{\text {cris }}(V)
$$

est l'application définie ci-dessus par $\Delta_{0}(\alpha(X))=\alpha(0)\left(\bmod (1-\varphi) \boldsymbol{D}_{\text {cris }}(V)\right)$, alors la suite exacte :

$$
0 \rightarrow \mathscr{D}(V)^{\Delta_{0}=0} \rightarrow \mathscr{D}(V) \stackrel{\Delta_{0}}{\rightarrow} \frac{\boldsymbol{D}_{\text {cris }}(V)}{(1-\varphi) \boldsymbol{D}_{\text {cris }}(V)} \rightarrow 0
$$

induit une suite exacte :

$$
0 \rightarrow \frac{\boldsymbol{D}_{\text {cris }}(V)}{(1-\varphi) \boldsymbol{D}_{\text {cris }}(V)} \rightarrow\left(\mathscr{D}(V)^{\Delta=0}\right)_{\Gamma_{n}} \rightarrow \mathscr{D}(V)_{\Gamma_{n}} \rightarrow \frac{\boldsymbol{D}_{\text {cris }}(V)}{(1-\varphi) \boldsymbol{D}_{\text {cris }}(V)} \rightarrow 0
$$

En comparant cette suite avec (3.1), on voit facilement que $\mathscr{D}(V) \underset{\Gamma_{n}}{\Delta_{0}=0}=\mathscr{D}(V) \stackrel{\text { (4.1) }}{\Gamma_{n}=0}$. Rappelons que pour $0 \leqslant i \leqslant p-2$, on note $\delta_{i}$ les idempotents de $\Delta_{K}=\operatorname{Gal}\left(K_{1} / K^{n}\right)$.

Lemme 4.8. (1) Le $\Lambda$-module $\left(X \mathbb{Z}_{p}[[X]]\right)^{\psi=0}$ est libre de rang 1 engendré par l'élément :

$$
z=\left(T \delta_{0}+\sum_{i=1}^{p-2} \delta_{i}\right)(1+X), \quad T=\gamma_{1}-1
$$

(2) Si $\boldsymbol{D}_{\text {cris }}(V) \simeq(1-\varphi) \boldsymbol{D}_{\text {cris }}(V) \oplus D^{\prime}$ est une décomposition de $\boldsymbol{D}_{\text {cris }}(V)$ en somme directe, alors :

$$
\mathscr{D}(V)^{\Delta_{0}=0}=\left(\mathbb{Z}_{p}[[X]]^{\psi=0} \otimes_{\mathbb{Z}_{p}}(1-\varphi) \boldsymbol{D}_{\text {cris }}(V)\right) \oplus\left(\left(X \mathbb{Z}_{p}[[X]]\right)^{\psi=0} \otimes_{\mathbb{Z}_{p}} D^{\prime}\right) .
$$

Preuve. Comme $\mathbb{Z}_{p}[[X]]^{\psi=0}$ est un $\Lambda$-module libre engendré par $(1+X)$, tout $f(X) \in \mathbb{Z}_{p}[[X]]^{\psi=0}$ s'écrit sous la forme $f(X)=\sum_{i=0}^{p-2} \delta_{i} g_{i}(T)(1+X)$, où $g_{i}(T) \in$ $\mathbb{Z}_{p}[[T]] \simeq \mathbb{Z}_{p}\left[\left[\Gamma_{1}\right]\right]$. Alors $f(0)=g_{0}(0)$, d'où on obtient que $f \in\left(X \mathbb{Z}_{p}[[X]]\right)^{\psi=0}$ si et seulement si $T$ divise $g_{0}(T)$. La première assertion s'en déduit.

Passons à la deuxième. Il est clair que pour tout $\alpha(X) \in(1-\varphi) \boldsymbol{D}_{\text {cris }}(V) \otimes_{\mathbb{Z}_{p}}$ $\mathbb{Z}_{p}[[X]]^{\psi=0}$ on a $\alpha(0) \in(1-\varphi) \boldsymbol{D}_{\text {cris }}(V)$, d'où $\Delta_{0}(\alpha)=0$. Si $\beta=d^{\prime} \otimes f(X) \in$ $D^{\prime} \otimes_{\mathbb{Z}_{p}} \mathbb{Z}_{p}[[X]]^{\psi=0}$, alors $\beta(0)=d^{\prime} f(0) \in D^{\prime}$ et donc $\Delta_{0}(\beta)=0$ si et seulement si $f(0)=0$ d'où $\left(\mathbb{Z}_{p}[[X]]^{\psi=0} \otimes_{\mathbb{Z}_{p}} D^{\prime}\right)^{\Delta_{0}=0}=\left(X \mathbb{Z}_{p}[[X]]\right)^{\psi=0} \otimes_{\mathbb{Z}_{p}} D^{\prime}$ et le lemme est démontré. 
Nous pouvons considérer $\tilde{\Xi}_{V, n}^{\varepsilon}$ comme un homomorphisme de $\mathscr{D}(V)^{\Delta_{0}=0}$ vers l'espace $\boldsymbol{D}_{\mathrm{dR}}^{K_{n}}(V) / \boldsymbol{D}_{\text {cris }}(V)^{\varphi=1}$.

Lemme 4.9. Pour tout $\alpha(X) \in \mathscr{D}(V)^{\Delta_{0}=0}$, on $a$ :

$\tilde{\Xi}_{V, n}^{\varepsilon}(\alpha(X))=p^{-n}\left(\sum_{k=1}^{n}(\sigma \otimes \varphi)^{-k} \alpha\left(\zeta_{p^{k}}-1\right)+(1-\varphi)^{-1} \alpha(0)\right)\left(\bmod \boldsymbol{D}_{\text {cris }}(V)^{\varphi=1}\right)$.

Preuve. Supposons d'abord que $\alpha(X) \in \mathscr{D}(V)^{\Delta=0}$. Dans ce cas, $\alpha(0) \in(1-$ $\varphi) \boldsymbol{D}_{\text {cris }}(V)$ et l'élément $(1-\varphi)^{-1} \alpha(0) \in \boldsymbol{D}_{\text {cris }}(V) / \boldsymbol{D}_{\text {cris }}(V)^{\varphi=1}$ est bien défini. Soit $F(X) \in \mathscr{H} \otimes_{K} \boldsymbol{D}_{\text {cris }}(V)$ telle que $(1-\varphi) F(X)=\alpha(X)$. Pour tout $0 \leqslant k \leqslant n-1$ on a :

$$
(\varphi \otimes \sigma)^{k} F\left(\zeta_{p^{n-k}}-1\right)-(\varphi \otimes \sigma)^{k+1} F\left(\zeta_{p^{n-k-1}}-1\right)=(\varphi \otimes \sigma)^{k} \alpha\left(\zeta_{p^{n-k}}-1\right)
$$

ainsi que $(1-\varphi) F(0)=\alpha(0)$, ce qui fait que :

$$
\begin{aligned}
\tilde{\Xi}_{V, n}^{\varepsilon}(\alpha(X)) & =p^{-n}(\varphi \otimes \sigma)^{-n} F\left(\zeta_{p^{n}}-1\right) \\
& =p^{-n}\left(\sum_{k=1}^{n}(\varphi \otimes \sigma)^{-k} \alpha\left(\zeta_{p^{k}}-1\right)+(1-\varphi)^{-1} \alpha(0)\right)
\end{aligned}
$$

$\left(\bmod \boldsymbol{D}_{\text {cris }}(V)^{\varphi=1}\right)$.

Passons maintenant au cas général. Si $\alpha(X) \in \mathscr{D}(V)^{\Delta_{0}=0}$, alors comme $\mathscr{D}(V)_{\Gamma_{n}}^{\Delta_{0}=0}=\mathscr{D}(V)_{\Gamma_{n}}^{\Delta=0}$, il existe $\beta(X) \in \mathscr{D}(V)^{\Delta=0}$ tel que $f(X)=\alpha(X)-\beta(X) \in$ $\left(\gamma_{n}-1\right) \mathscr{D}(V)^{\Delta_{0}=0}$.

Comme $\sum_{k=1}^{n}(\sigma \otimes \varphi)^{-k} f\left(\zeta_{p^{k}}-1\right)+(1-\varphi)^{-1} \alpha(0) \equiv 0\left(\bmod \boldsymbol{D}_{\text {cris }}(V)^{\varphi=1}\right)$, on obtient :

$$
\begin{aligned}
& \tilde{\Xi}_{V, n}^{\varepsilon}(\alpha(X))=\tilde{\Xi}_{V, n}^{\varepsilon}(\beta(X)) \\
& \quad=p^{-n}\left(\sum_{k=1}^{n}(\sigma \otimes \varphi)^{-k} \alpha\left(\zeta_{p^{k}}-1\right)+(1-\varphi)^{-1} \alpha(0)\right) \quad\left(\bmod \boldsymbol{D}_{\text {cris }}(V)^{\varphi=1}\right) .
\end{aligned}
$$

Pour tout caractère $\eta \in X\left(G_{n}\right)$ on note

$$
\tilde{\boldsymbol{\Xi}}_{V, \eta}^{\varepsilon}: \mathscr{D}(V)_{\eta}^{\Delta_{0}=0} \rightarrow \boldsymbol{D}_{\mathrm{dR}}^{K_{n}}(V)_{\eta} / \boldsymbol{D}_{\text {cris }}(V)_{\eta}^{\varphi=1}
$$

la $\eta$-composante de $\tilde{\Xi}_{V, n}^{\varepsilon}: \mathscr{D}(V)_{\Gamma_{n}}^{\Delta_{0}} \rightarrow \boldsymbol{D}_{\mathrm{dR}}^{K_{n}}(V) / \boldsymbol{D}_{\text {cris }}(V)^{\varphi=1}$. Bien sûr, on a $\boldsymbol{D}_{\text {cris }}(V)_{\eta}^{\varphi=1}=0$ si $\eta \neq \eta_{0}$. 
Corollaire 4.10. Si $\alpha=(1+X) \otimes d \in \mathbb{Z}_{p}[[X]]^{\psi=0} \otimes_{\mathbb{Z}_{p}}\left((1-\varphi) \boldsymbol{D}_{\text {cris }}(V)\right)$ et si $\eta \in X\left(G_{n}\right)$ est un caractère de conducteur $p^{k}$, alors :

$$
\tilde{\Xi}_{V, \eta}^{\varepsilon}(\alpha)= \begin{cases}p^{-n} \varphi^{-k}(d) e_{\eta}\left(\zeta_{p^{k}}\right), & \text { si } \eta \neq \eta_{0}, \\ \frac{1}{\left[K_{n}: K\right]}\left(1-p^{-1} \varphi^{-1}\right)(1-\varphi)^{-1}(d), & \text { si } \eta=\eta_{0} .\end{cases}
$$

Parailleurs, si $\beta=z \otimes d^{\prime} \in\left(X \mathbb{Z}_{p}[[X]]\right)^{\psi=0} \otimes D^{\prime}$ et si $X\left(G_{n}\right) \simeq X\left(\Delta_{K}\right) \times X\left(\Gamma_{1} / \Gamma_{n}\right)$ est la décomposition du groupe des caractères de $G_{n}$ qui correspond à l'isomorphisme canonique $G_{n} \simeq \Delta_{K} \times\left(\Gamma_{1} / \Gamma_{n}\right)$, alors :

$$
\tilde{\Xi}_{V, \eta}^{\varepsilon}(\beta)= \begin{cases}p^{-n} \varphi^{-k}\left(d^{\prime}\right) e_{\eta}\left(\zeta_{p^{k}}\right) & \text { si } \eta \notin X\left(\Gamma_{1} / \Gamma_{n}\right), \\ p^{-n}\left(\eta\left(\gamma_{1}\right)-1\right) \varphi^{-k}\left(d^{\prime}\right) e_{\eta}\left(\zeta_{p^{k}}\right) & \text { si } \eta \in X\left(\Gamma_{1} / \Gamma_{n}\right), \eta \neq \eta_{0}, \\ 0 & \text { si } \eta=\eta_{0} .\end{cases}
$$

Preuve. Si $\eta \neq \eta_{0}$ est un caractère de conducteur $p^{k}$, alors $e_{\eta}\left(\zeta_{p^{m}}\right)=0$ si $m \neq k$, d'où $\tilde{\Xi}_{V, \eta}^{\varepsilon}(\alpha)=p^{-n} \varphi^{-k}(d) e_{\eta}\left(\zeta_{p^{k}}\right)$. D'autre part, on a :

$$
e_{\eta_{0}}\left(\zeta_{p^{m}}\right)= \begin{cases}0 & \text { si } 2 \leqslant m \leqslant n \\ \frac{1}{1-p} & \text { si } m=1\end{cases}
$$

d'où :

$$
\begin{aligned}
\tilde{\Xi}_{V, \eta_{0}}^{\varepsilon}(\alpha) & =p^{-n}\left(\varphi^{-1}(d)(1-p)^{-1}+(1-\varphi)^{-1}(d)\right) \\
& =\frac{(1-\varphi)^{-1}}{p^{n}(1-p)}\left(\varphi^{-1}-p\right)(d) \\
& =\frac{1}{p^{n-1}(p-1)}(1-\varphi)^{-1}\left(1-p^{-1} \varphi^{-1}\right)(d) \quad\left(\bmod \boldsymbol{D}_{\text {cris }}(V)^{\varphi=1}\right) .
\end{aligned}
$$

Ceci montre la première assertion; passons à la seconde. Soit $\eta=\delta_{i} \eta^{\prime}$, où $\eta^{\prime} \in X\left(\Gamma_{1} / \Gamma_{n}\right)$. Si $\eta \notin X\left(\Gamma_{1} / \Gamma_{n}\right)$, alors $i \neq 0, e_{\eta}(z)=e_{\eta}(1+X)$ et le calcul déjà fait ci-dessus donne $\tilde{\Xi}_{V, \eta}^{\varepsilon}(\beta)=p^{-n} \varphi^{-k}\left(d^{\prime}\right) e_{\eta}\left(\zeta_{p^{k}}\right)$. Si $\eta \in X\left(\Gamma_{1} / \Gamma_{n}\right)$, alors $e_{\eta}(z)=e_{\eta}\left(\left(\gamma_{1}-1\right)(1+X)\right)=\left(\eta\left(\gamma_{1}\right)-1\right) e_{\eta}(1+X)$, d'où $\tilde{\Xi}_{V, \eta}^{\varepsilon}(\beta)=$ $p^{-n}\left(\eta\left(\gamma_{1}\right)-1\right) \varphi^{-k}\left(d^{\prime}\right) e_{\eta}\left(\zeta_{p^{k}}\right)$. En particulier, si $\eta=\eta_{0}$ cette formule donne $\tilde{\Xi}_{V, \eta_{0}}^{\varepsilon}(\beta)=0$ et le corollaire est démontré.

Le (2) de la proposition 3.2 donne une suite exacte :

$0 \rightarrow \operatorname{ker} \tilde{\Xi}_{V, n}^{\varepsilon} \rightarrow \mathscr{D}(V)_{\Gamma_{n}}^{\Delta_{0}=0} \stackrel{\hat{\Xi}_{V, n}^{\varepsilon}}{\longrightarrow} \frac{\boldsymbol{D}_{\mathrm{dR}}^{K_{n}}(V)}{\boldsymbol{D}_{\text {cris }}(V)^{\varphi=1}} \rightarrow \frac{\boldsymbol{D}_{\text {cris }}(V)}{\left(1-p^{-1} \varphi^{-1}\right) \boldsymbol{D}_{\text {cris }}(V)} \rightarrow 0$. 
En composant cette suite avec les suites tautologiques :

$$
\begin{aligned}
& 0 \rightarrow \boldsymbol{D}_{\text {cris }}(V)^{\varphi=1} \rightarrow \boldsymbol{D}_{\text {cris }}(V) \stackrel{1-\varphi}{\longrightarrow} \boldsymbol{D}_{\text {cris }}(V) \rightarrow \frac{\boldsymbol{D}_{\text {cris }}(V)}{(1-\varphi) \boldsymbol{D}_{\text {cris }}(V)} \rightarrow 0, \\
& 0 \rightarrow \boldsymbol{D}_{\text {cris }}(V)^{\varphi=p^{-1}} \rightarrow \boldsymbol{D}_{\text {cris }}(V) \\
& \stackrel{1-p^{-1} \varphi^{-1}}{\longrightarrow} \boldsymbol{D}_{\text {cris }}(V) \rightarrow \frac{\boldsymbol{D}_{\text {cris }}(V)}{\left(1-p^{-1} \varphi^{-1}\right) \boldsymbol{D}_{\text {cris }}(V)} \rightarrow 0
\end{aligned}
$$

et en utilisant le (1) de la proposition 3.2, on obtient un isomorphisme canonique :

$$
\begin{aligned}
\kappa_{V, n}: \operatorname{det}_{\mathbb{Q}_{p}\left[G_{n}\right]} \mathcal{D}(V)_{\Gamma_{n}}^{\Delta=0} \otimes_{\mathbb{Q}_{p}\left[G_{n}\right]} \operatorname{det}_{\mathbb{Q}_{p}\left[G_{n}\right]}^{-1} D_{\mathrm{dR}}^{K_{n}}(V) \\
\simeq\left(\operatorname{det}_{\mathbb{Q}_{p}\left[G_{n}\right]}^{-1} \boldsymbol{D}_{\text {cris }}(V)^{\varphi=1} \operatorname{det}_{\mathbb{Q}_{p}\left[G_{n}\right]} \frac{\boldsymbol{D}_{\text {cris }}(V)}{(1-\varphi) \boldsymbol{D}_{\text {cris }}(V)}\right) \\
\quad \otimes\left(\operatorname{det}_{\mathbb{Q}_{p}\left[G_{n}\right]} \boldsymbol{D}_{\text {cris }}(V)^{\varphi=p^{-1}} \otimes \operatorname{det}_{\mathbb{Q}_{p}\left[G_{n}\right]}^{-1} \frac{\boldsymbol{D}_{\text {cris }}(V)}{\left(1-p^{-1} \varphi^{-1}\right) \boldsymbol{D}_{\text {cris }}(V)}\right) \\
\simeq \mathbb{Q}_{p}\left[G_{n}\right]
\end{aligned}
$$

Rappelons que l'on note $R_{n}$ le $\mathcal{O}_{K}\left[G_{n}\right]$-module libre engendré par $x_{n}=\zeta_{p^{n}}+$ $\zeta_{p^{n-1}}+\cdots+\zeta_{p}$. On fixe un réseau $M$ de $\boldsymbol{D}_{\text {cris }}(V)$ et l'on pose $\mathscr{D}_{M}(V)=\mathcal{O}_{K}[[X]]_{\psi} \otimes$ $M$ et $M_{n}=R_{n} \otimes_{\mathbb{Z}_{p}} M$.

Proposition 4.11. L'isomorphisme $\kappa_{V, n}$ envoie :

$$
\operatorname{det}_{\mathbb{Z}_{p}\left[G_{n}\right]} \mathcal{D}_{M}(V)_{\Gamma_{n}}^{\Delta_{0}=0} \otimes_{\mathbb{Z}_{p}\left[G_{n}\right]} \operatorname{det}_{\mathbb{Z}_{p}\left[G_{n}\right]}^{-1} M_{n}
$$

sur le réseau engendré par:

$$
\begin{aligned}
q_{K}^{-n d} \sum_{\eta \neq \eta_{0}}\left(\eta\left(\gamma_{1}\right)-1\right)^{\delta(\eta) \operatorname{dim}_{\mathbb{Q}_{p}} \boldsymbol{D}_{\text {cris }}(V)^{\varphi=1}} \operatorname{det}\left(\varphi \mid \boldsymbol{D}_{\text {cris }}(V)\right)^{-a(\eta)} e_{\eta}+ \\
(-1)^{f d}\left(1-\frac{1}{p}\right)^{\operatorname{dim}_{\mathbb{Q}_{p}} \boldsymbol{D}_{\text {cris }}(V)^{\varphi=p^{-1}}} p^{(1-n) f d+\operatorname{dim}_{\mathbb{Q}_{p}} \boldsymbol{D}_{\text {cris }}(V)^{\varphi=1}} e_{\eta_{0}},
\end{aligned}
$$

où $\delta(\eta)=1$ si $\eta \in X\left(\Gamma_{1}\right)$ et $\delta(\eta)=0$ sinon.

Preuve. Si $N=(1-\varphi) \boldsymbol{D}_{\text {cris }}(V) \cap M$, alors $M / N$ est sans torsion et il existe $N^{\prime} \subset M$ tel que $M=N \oplus N^{\prime}$. Par le lemme 4.8, on a :

$$
\left.\mathscr{D}_{M}(V)^{\Delta_{0}=0}=\left(\mathbb{Z}_{p}[[X]]^{\psi=0} \otimes_{\mathbb{Z}_{p}} N\right) \oplus\left(X \mathbb{Z}_{p}[[X]]\right)^{\psi=0} \otimes_{\mathbb{Z}_{p}} N^{\prime}\right) .
$$

En particulier $\mathscr{D}_{M}(V)^{\Delta_{0}=0}$ est $\Lambda$-libre et par le lemme 2.6, il suffit de démontrer la proposition caractère par caractère. 
On fixe une base $\left(n_{i}\right)$ (resp. $\left.\left(n_{j}^{\prime}\right)\right)$ de $N$ (resp. $\left.N^{\prime}\right)$. Les éléments $\alpha_{i}=(1+X) \otimes n_{i}$ forment une base de $\mathbb{Z}_{p}[[X]]^{\psi=0} \otimes_{\mathbb{Z}_{p}} N$ et les éléments $\alpha_{j}^{\prime}=z \otimes n_{j}^{\prime}$ forment une base de $\left(X \mathbb{Z}_{p}[[X]]\right)^{\psi=0} \otimes_{\mathbb{Z}_{p}} N^{\prime}$. On pose :

$$
\begin{aligned}
\tilde{\alpha} & =\wedge_{i} \alpha_{i} \in \operatorname{det}_{\Lambda}\left(\mathbb{Z}_{p}[[X]]^{\psi=0} \otimes_{\mathbb{Z}_{p}} N\right), \\
\tilde{\alpha}^{\prime} & =\wedge_{j} \alpha_{j}^{\prime} \in \operatorname{det}_{\Lambda}\left(\left(X \mathbb{Z}_{p}[[X]]\right)^{\psi=0} \otimes_{\mathbb{Z}_{p}} N^{\prime}\right), \\
\tilde{a} & =\tilde{\alpha} \wedge \tilde{\alpha}^{\prime} \in \operatorname{det}_{\Lambda} \mathcal{D}_{M}(V)^{\Delta_{0}=0} .
\end{aligned}
$$

On pose aussi $\beta_{i}=x_{n} \otimes n_{i}$ et $\beta_{j}^{\prime}=x_{n} \otimes n_{j}^{\prime}$. Alors $\left(\beta_{i}\right)$ (resp. $\left.\left(\beta_{j}^{\prime}\right)\right)$ est une base de $R_{n} \otimes_{\mathbb{Z}_{p}} N$ (resp. $R_{n} \otimes_{\mathbb{Z}_{p}} N^{\prime}$ ). On pose :

$$
\begin{aligned}
\tilde{\beta} & =\wedge_{i} \beta_{i} \in \operatorname{det}_{\Lambda}\left(R_{n} \otimes_{\mathbb{Z}_{p}} N\right), \\
\tilde{\beta}^{\prime} & =\wedge_{j} \beta_{j}^{\prime} \in \operatorname{det}_{\Lambda}\left(R_{n} \otimes_{\mathbb{Z}_{p}} N^{\prime}\right), \\
\tilde{b} & =\tilde{\beta} \wedge \tilde{\beta}^{\prime} \in \operatorname{det}_{\Lambda}\left(R_{n} \otimes_{\mathbb{Z}_{p}} M\right) .
\end{aligned}
$$

Soit $\eta$ un caractère non-trivial de $G_{n}$ de conducteur $p^{k}$. Les suites exactes (1) et (3) de la proposition 3.2 impliquent que la $\eta$-composante de $\tilde{\Xi}_{V, n}^{\varepsilon}$ est un isomorphisme $\tilde{\Xi}_{V, \eta}^{\varepsilon}: \mathscr{D}(V)_{\eta}^{\Delta_{0}=0} \stackrel{\sim}{\rightarrow} D_{\mathrm{dR}}^{K_{n}}(V)_{\eta}$ et que $\kappa_{V, \eta}=\operatorname{det}\left(\tilde{\Xi}_{V, \eta}^{\varepsilon}\right)$. On a $e_{\eta}\left(\beta_{i}\right)=e_{\eta}\left(\zeta_{p^{k}}\right) n_{i}$, $e_{\eta}\left(\beta_{j}^{\prime}\right)=e_{\eta}\left(\zeta_{p^{k}}\right) n_{j}^{\prime}$. Si $\eta \notin X\left(\Gamma_{1} / \Gamma_{n}\right)$, alors le corollaire 4.10 donne :

$$
\begin{aligned}
& \tilde{\Xi}_{V, \eta}^{\varepsilon}\left(\alpha_{i}\right)=p^{-n} \varphi^{-k}\left(n_{i}\right) e_{\eta}\left(\zeta_{p^{k}}\right), \\
& \tilde{\Xi}_{V, \eta}^{\varepsilon}\left(\alpha_{j}^{\prime}\right)=p^{-n} \varphi^{-k}\left(n_{j}^{\prime}\right) e_{\eta}\left(\zeta_{p^{k}}\right) .
\end{aligned}
$$

On en déduit que :

$$
\kappa_{V, \eta}\left(\tilde{a}_{\eta} \otimes \tilde{b}_{\eta}^{-1}\right)=p^{-n \operatorname{dim}_{\mathbb{Q}_{p}} \boldsymbol{D}_{\text {cris }}(V)} \operatorname{det}_{\mathbb{Q}_{p}}\left(\varphi^{-k} \mid \boldsymbol{D}_{\text {cris }}(V)\right) .
$$

Si $\eta \in X\left(\Gamma_{1} / \Gamma_{n}\right)$ est un caractère non-trivial, alors encore par le corollaire 4.10 on a $\tilde{\Xi}_{V, n, \eta}^{\varepsilon}\left(\alpha_{j}^{\prime}\right)=p^{-n}\left(\eta\left(\gamma_{1}\right)-1\right) \varphi^{-k}\left(n_{j}^{\prime}\right) e_{\eta}\left(\zeta_{p^{k}}\right)$, d'où :

$\kappa_{V, \eta}\left(\tilde{a}_{\eta} \otimes \tilde{b}_{\eta}^{-1}\right)=p^{-n \operatorname{dim}_{\mathbb{Q}_{p}} \boldsymbol{D}_{\text {cris }}(V)}\left(\eta\left(\gamma_{1}\right)-1\right)^{\operatorname{dim}_{\mathbb{Q}_{p}} \boldsymbol{D}_{\text {cris }}(V)^{\varphi=1}} \operatorname{det}_{\mathbb{Q}_{p}}\left(\varphi^{-k} \mid \boldsymbol{D}_{\text {cris }}(V)\right)$.

Pour terminer la preuve il reste à étudier le cas $\eta=\eta_{0}$. Dans ce cas, le corollaire 4.10 donne :

$$
\begin{aligned}
& \tilde{\Xi}_{V, \eta_{0}}^{\varepsilon}\left(\alpha_{i}\right)=\frac{1}{\left[K_{n}: K\right]}\left(1-p^{-1} \varphi^{-1}\right)(1-\varphi)^{-1} n_{i}, \\
& \tilde{\Xi}_{V, \eta_{0}}^{\varepsilon}\left(\alpha_{j}^{\prime}\right)=0 .
\end{aligned}
$$

Pour simplifier les formules, nous identifions $\mathscr{D}(V)$ avec $\Lambda \otimes \boldsymbol{D}_{\text {cris }}(V)$ via l'isomorphisme canonique $\mathbb{Z}_{p}[[X]]^{\psi=0} \simeq \Lambda$ qui envoie $(1+X)$ sur 1 . On décompose 
$\Lambda$ en somme directe $: \Lambda \simeq \bigoplus_{i=0}^{p-2} \delta_{i} \mathbb{Z}_{p}\left[\left[\Gamma_{1}\right]\right], \mathbb{Z}_{p}\left[\left[\Gamma_{1}\right]\right] \simeq \mathbb{Z}_{p}[[T]]$. On utilise l'isomorphisme $\eta_{0}\left(\mathbb{Q}_{p}\left[G_{n}\right]\right)=\mathbb{Q}_{p} \eta_{0} \simeq \mathbb{Q}_{p}$ pour identifier $\mathcal{D}(V)_{\eta_{0}}$ avec $\boldsymbol{D}_{\text {cris }}(V)$. En prenant la $\eta_{0}$-composante de la suite (4.1), on obtient une suite exacte :

$$
0 \rightarrow \frac{\boldsymbol{D}_{\text {cris }}(V)}{(1-\varphi) \boldsymbol{D}_{\text {cris }}(V)} \rightarrow \mathscr{D}(V)_{\eta_{0}}^{\Delta_{0}=0} \rightarrow(1-\varphi) \boldsymbol{D}_{\text {cris }}(V) \rightarrow 0 .
$$

Si $\lambda=T \delta_{0}+\sum_{i=1}^{p-2} \delta_{i} \in \Lambda$, alors $\mathscr{D}(V)^{\Delta_{0}=0} \simeq\left(\Lambda \otimes_{\mathbb{Z}_{p}}(1-\varphi) \boldsymbol{D}_{\text {cris }}(V)\right) \oplus$ $\left(\lambda \Lambda \otimes_{\mathbb{Z}_{p}} D^{\prime}\right)$, ce qui permet d'identifier $\mathscr{D}(V)_{\eta_{0}}^{\Delta_{0}=0}$ avec $(1-\varphi) \boldsymbol{D}_{\text {cris }}(V) \oplus D^{\prime}$. La deuxième flèche de la suite (4.5) est donnée par la formule $d^{\prime} \mapsto e_{\eta_{0}}\left(\gamma_{n}-1\right) \otimes d^{\prime}$ $(\operatorname{voir}(3.1))$. Posons $g_{n}(T)=\left((1+T)^{p^{n-1}}-1\right) / T$. Alors $\gamma_{n}-1=\delta_{0} T g_{n}(T)$ et comme $\delta_{0} T=\delta_{0} \lambda$, on obtient :

$$
e_{\eta_{0}}\left(\gamma_{n}-1\right)=\lambda e_{\eta_{0}} g_{n}(T)=\lambda g_{n}(0) e_{\eta_{0}}=p^{n-1} \lambda e_{\eta_{0}} .
$$

On en déduit que la suite (4.5) est isomorphe à la suite :

$$
0 \rightarrow D^{\prime} \rightarrow(1-\varphi) \boldsymbol{D}_{\text {cris }}(V) \oplus D^{\prime} \rightarrow(1-\varphi) \boldsymbol{D}_{\text {cris }}(V) \rightarrow 0,
$$

dont les flèches sont données par les formules $d^{\prime} \mapsto\left(0, p^{n-1} d^{\prime}\right)$ et $(a, b) \mapsto a$.

Les suites exactes (4.2), (4.5) et la suite (1) de la proposition 3.2 s'insèrent dans un diagramme :

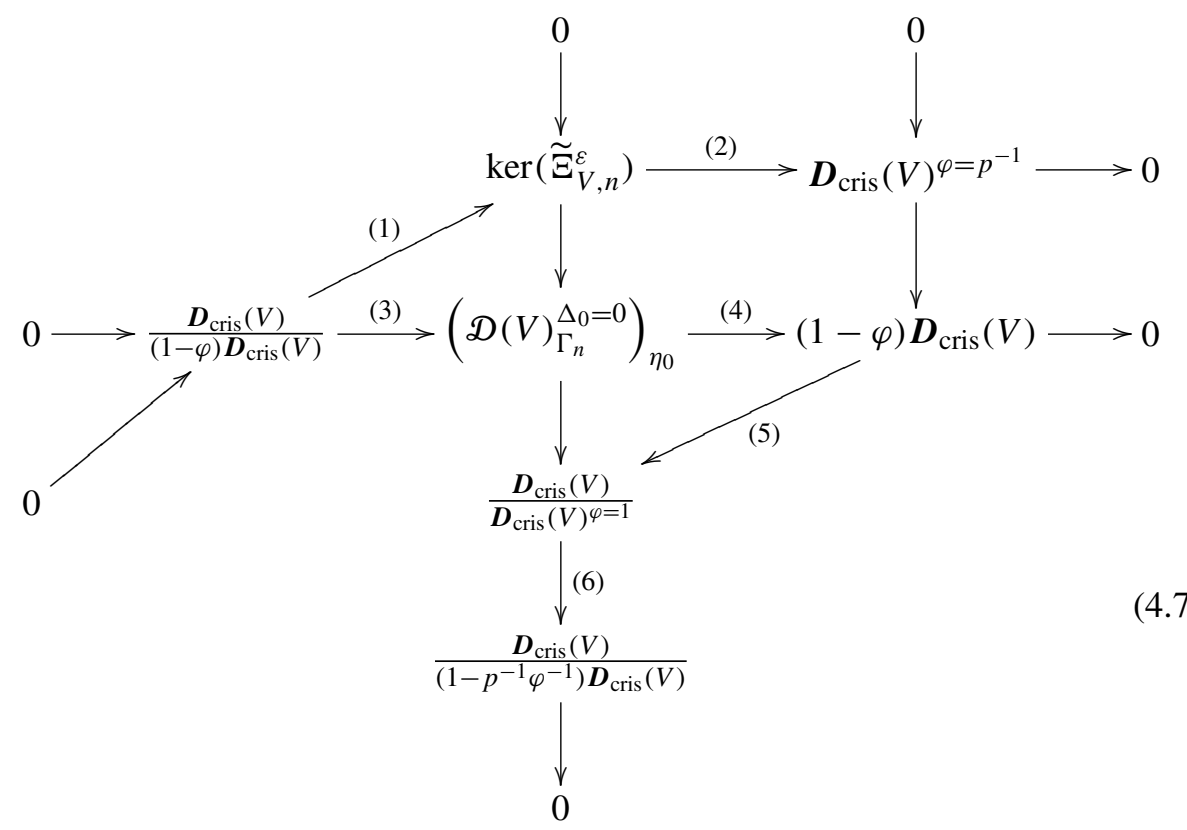


dont les flèches sont données par les formules suivantes :

(1) et (3) : $\quad d \mapsto d \otimes\left(\gamma_{n}-1\right)(1+X)$,

(2) et (4) : $\quad \alpha(X) \mapsto \alpha(0)$,

(5) : $\quad d \mapsto \frac{1}{\left[K_{n}: K\right]}\left(1-p^{-1} \varphi^{-1}\right)(1-\varphi)^{-1}(d)\left(\bmod \boldsymbol{D}_{\text {cris }}(V)^{\varphi=1}\right)$,

(6) : $\quad d \mapsto\left[K_{n}: K\right] d$.

La commutativité de ce diagramme est immédiate.

Posons $\tilde{n}=\wedge_{i} n_{i} \in \operatorname{det}_{\mathbb{Z}_{p}} N$ et $\tilde{n}^{\prime}=\wedge_{j} n_{j}^{\prime} \in \operatorname{det}_{\mathbb{Z}_{p}} N^{\prime}$, ce qui fait que $\tilde{n} \otimes \tilde{n}^{\prime}$ est une base de $\operatorname{det}_{\mathbb{Z}_{p}} M$. Comme $e_{\eta_{0}}\left(x_{n}\right)=e_{\eta_{0}}\left(\zeta_{p}\right)=(1-p)^{-1}$, on a $\tilde{b}_{\eta_{0}}=$ $(1-p)^{-\operatorname{dim}_{\mathbb{Q}_{p}} \boldsymbol{D}_{\text {cris }}(V)}\left(\tilde{n} \otimes \tilde{n}^{\prime}\right)$ et la $\eta_{0}$-composante de la formule à montrer s'écrit :

$$
\begin{aligned}
\kappa_{V, \eta_{0}} & \left(\tilde{\alpha}_{\eta_{0}} \otimes\left(\tilde{n} \otimes \tilde{n}^{\prime}\right)^{-1}\right) \\
& =(p-1)^{-f d}\left(1-\frac{1}{p}\right)^{\operatorname{dim}_{\mathbb{Q}_{p}} D_{\text {cris }}(V)^{\varphi=p^{-1}}} p^{(1-n) f d+\operatorname{dim}_{\mathbb{Q}_{p}} D_{\text {cris }}(V)^{\varphi=1}} .
\end{aligned}
$$

Fixons des bases $\tilde{m}_{1} \in \operatorname{det}_{\mathbb{Z}_{p}} M^{\varphi=1}$ et $\tilde{m}_{2} \in \operatorname{det}_{\mathbb{Z}_{p}}\left(M / M^{\varphi=1}\right)$ telles que $\tilde{m}_{1} \otimes \tilde{m}_{2} \simeq$ $\tilde{n} \otimes \tilde{n}^{\prime}$. Rappelons que l'isomorphisme $\mathscr{D}(V)_{\eta_{0}}^{\Delta_{0}=0}$ identifie $\tilde{\alpha}_{\eta_{0}}$ avec $\tilde{n} \otimes \tilde{n}^{\prime}$. Considérons les isomorphismes canoniques :

$$
\begin{aligned}
i_{1}: \operatorname{det}_{\mathbb{Q}_{p}}\left(\frac{\boldsymbol{D}_{\text {cris }}(V)}{(1-\varphi) \boldsymbol{D}_{\text {cris }}(V)}\right) \otimes \operatorname{det}_{\mathbb{Q}_{p}}^{-1} \boldsymbol{D}_{\text {cris }}(V)^{\varphi=1} \\
\stackrel{\text { via }(4.4)}{\longrightarrow} \operatorname{det}_{\mathbb{Q}_{p}} \boldsymbol{D}_{\text {cris }}(V) \otimes \operatorname{det}_{\mathbb{Q}_{p}}^{-1} \boldsymbol{D}_{\text {cris }}(V) \stackrel{\text { id }}{\rightarrow} \mathbb{Q}_{p}
\end{aligned}
$$

et

$$
\begin{aligned}
i_{2}: \operatorname{det}_{\mathbb{Q}_{p}}\left((1-\varphi) \boldsymbol{D}_{\text {cris }}(V)\right) \otimes \operatorname{det}_{\mathbb{Q}_{p}}^{-1}\left(\frac{\boldsymbol{D}_{\text {cris }}(V)}{\boldsymbol{D}_{\text {cris }}(V)^{\varphi=1}}\right) \\
\stackrel{\text { via (4.7) }}{\longrightarrow} \operatorname{det}_{\mathbb{Q}_{p}} \boldsymbol{D}_{\text {cris }}(V)^{\varphi=p^{-1}} \otimes \operatorname{det}_{\mathbb{Q}_{p}^{-1}}^{-1}\left(\frac{\boldsymbol{D}_{\text {cris }}(V)}{\left(1-p^{-1} \varphi^{-1}\right) \boldsymbol{D}_{\text {cris }}(V)}\right) \\
\stackrel{\text { via }(4.4)}{\longrightarrow} \operatorname{det}_{\mathbb{Q}_{p}} \boldsymbol{D}_{\text {cris }}(V) \otimes \operatorname{det}_{\mathbb{Q}_{p}}^{-1} \boldsymbol{D}_{\text {cris }}(V) \stackrel{\text { id }}{\rightarrow} \mathbb{Q}_{p} .
\end{aligned}
$$

Par définition, l'application $\kappa_{V, \eta_{0}}$ s'obtient en trivialisant la suite exacte verticale du diagramme (4.7) via la suite exacte (1) de la proposition 3.2 et les suites exactes tautologiques (4.4). En utilisant la commutativité du diagramme (4.7) et la suite (4.6) on obtient, grâce à la fonctorialité des déterminants :

$$
\kappa_{V, \eta_{0}}\left(\tilde{\alpha}_{\eta_{0}} \otimes\left(\tilde{n} \otimes \tilde{n}^{\prime}\right)^{-1}\right)=p^{(1-n) \operatorname{dim}_{\mathbb{Q}_{p}} \boldsymbol{D}_{\text {cris }}(V)^{\varphi=1}} i_{1}\left(\tilde{n}^{\prime} \otimes \tilde{m}_{1}^{-1}\right) i_{2}\left(\tilde{n} \otimes \tilde{m}_{2}^{-1}\right) .
$$


L'isomorphisme $1-\varphi: \boldsymbol{D}_{\text {cris }}(V) / \boldsymbol{D}_{\text {cris }}(V)^{\varphi=1} \simeq(1-\varphi) \boldsymbol{D}_{\text {cris }}(V)$ induit un isomorphisme $\operatorname{det}_{\mathbb{Q}_{p}}\left(\frac{\boldsymbol{D}_{\text {cris }}(V)}{\boldsymbol{D}_{\text {cris }}(V)^{\varphi=1}}\right) \simeq \operatorname{det}_{\mathbb{Q}_{p}}(1-\varphi) \boldsymbol{D}_{\text {cris }}(V)$ et on note $\left[\tilde{n}:(1-\varphi) \tilde{m}_{2}\right]$ l'élément de $\mathbb{Q}_{p}$ défini par $(1-\varphi) \tilde{m}_{2}=\left[\tilde{n}:(1-\varphi) \tilde{m}_{2}\right] \tilde{n}$. La définition de $i_{1}$ implique alors directement :

$$
i_{1}\left(\tilde{n}^{\prime} \otimes \tilde{m}_{1}^{-1}\right)=\left[\tilde{n}:(1-\varphi) \tilde{m}_{2}\right] .
$$

Pour calculer $i_{2}\left(\tilde{n} \otimes \tilde{m}_{2}^{-1}\right)$, considérons le diagramme tautologique suivant :

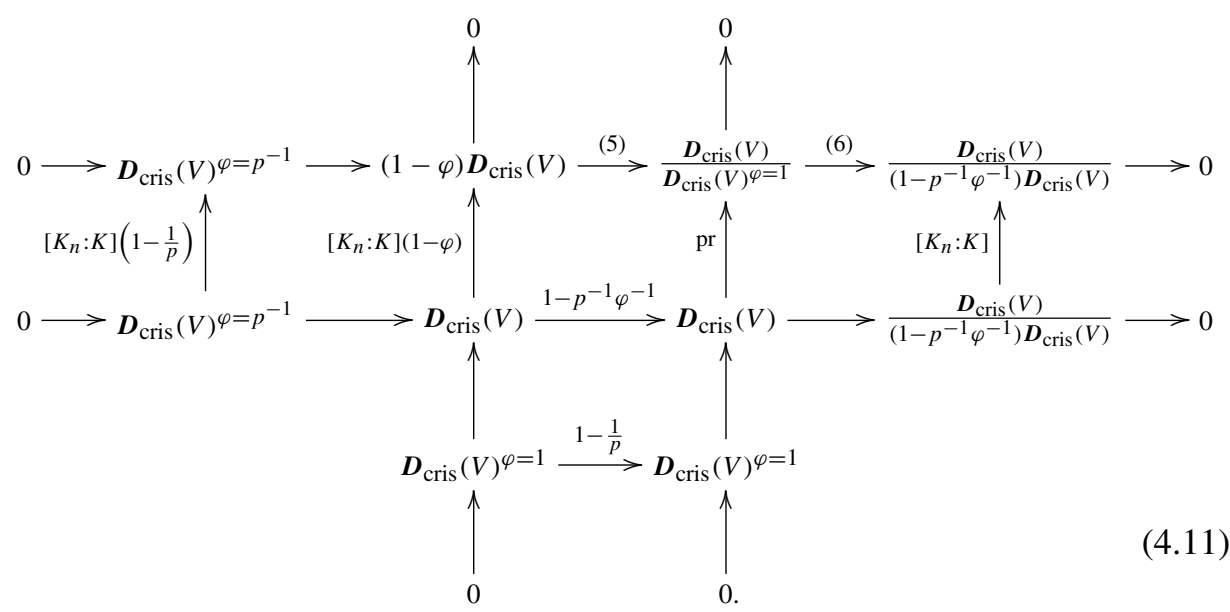

Ce diagramme fournit un diagramme commutatif :

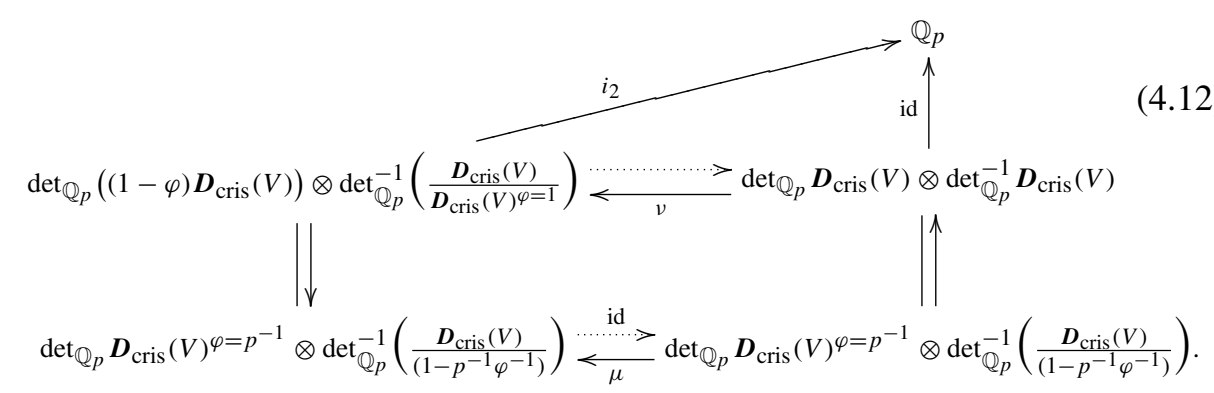

Les isomorphismes verticaux de ce diagramme sont induits par les lignes exactes du diagramme (4.11). Par définition, $i_{2}$ est le composé de ces isomorphismes avec la flèche inférieure pointillée et l'isomorphisme id. Les isomorphismes $v$ et $\mu$ sont induits par les flèches verticales de (4.11) qui envoient la deuxième ligne de ce diagramme sur la première. En particulier, on voit tout de suite que $\mu$ coïncide avec la multiplication par $(1-1 / p)^{\operatorname{dim} \boldsymbol{D}_{\text {cris }}(V)^{\varphi=p^{-1}}}$. D'autre part, $\left[K_{n}: K\right]=p^{n-1}(p-1)$ 
et on a :

$$
\begin{aligned}
& v\left(\left(\tilde{m}_{1} \otimes \tilde{m}_{2}\right) \otimes\left(\tilde{m}_{1} \otimes \tilde{m}_{2}\right)^{-1}\right) \\
& =\left(p^{n-1}(p-1)\right)^{\operatorname{dim}_{\mathbb{Q}_{p}}\left(\boldsymbol{D}_{\text {cris }}(V) / \boldsymbol{D}_{\text {cris }}(V)^{\varphi=1}\right.} \\
& \quad \operatorname{det}\left(1-1 / p \mid \boldsymbol{D}_{\text {cris }}(V)^{\varphi=1}\right)(1-\varphi) \tilde{m}_{2} \otimes \tilde{m}_{2}^{-1} .
\end{aligned}
$$

En utilisant la commutativité de (4.12) on en déduit que :

$$
\begin{aligned}
& i_{2}\left((1-\varphi) \tilde{m}_{2} \otimes \tilde{m}_{2}^{-1}\right) \\
& \quad=\left(1-\frac{1}{p}\right)^{\operatorname{dim}_{\mathbb{Q}_{p}} \boldsymbol{D}_{\text {cris }}(V)^{\varphi=p^{-1}}-\operatorname{dim}_{\mathbb{Q}_{p}} \boldsymbol{D}_{\text {cris }}(V)^{\varphi=1}}\left(\frac{p^{1-n}}{p-1}\right)^{\operatorname{dim}_{\mathbb{Q}_{p}}\left(\boldsymbol{D}_{\text {cris }}(V) / \boldsymbol{D}_{\text {cris }}(V)^{\varphi=1}\right.} .
\end{aligned}
$$

Comme $i_{2}\left(\tilde{n} \otimes \tilde{m}_{2}^{-1}\right)=\left[\tilde{n}:(1-\varphi) \tilde{m}_{2}\right]^{-1} i_{2}\left((1-\varphi) \tilde{m}_{2} \otimes \tilde{m}_{2}^{-1}\right)$, en mettant ensemble (4.13), (4.10) et (4.9) on obtient (4.8) ce qui termine la démonstration de la proposition 4.11 .

Proposition 4.12. L'image de $\operatorname{det}_{\mathbb{Z}_{p}\left[G_{n}\right]} \mathcal{D}_{M}(V)_{\Gamma_{n}} \otimes_{\mathbb{Z}_{p}\left[G_{n}\right]} \operatorname{det}_{\mathbb{Z}_{p}\left[G_{n}\right]}^{-1} M_{n}$ dans $\mathbb{Q}_{p}\left[G_{n}\right]$ est engendrée par

$$
\begin{aligned}
& q_{K}^{-n d} \sum_{\eta \neq \eta_{0}} \operatorname{det}\left(\varphi \mid \boldsymbol{D}_{\text {cris }}(V)\right)^{-a(\eta)} e_{\eta} \\
& \quad+(-1)^{f d}\left(1-\frac{1}{p}\right)^{\operatorname{dim}_{\mathbb{Q}_{p}} \boldsymbol{D}_{\text {cris }}(V)^{\varphi=p^{-1}}} p^{(1-n) f d+n \operatorname{dim}_{\mathbb{Q}_{p}} \boldsymbol{D}_{\text {cris }}(V)^{\varphi=1}} e_{\eta_{0}} .
\end{aligned}
$$

Preuve. Il suffit de calculer l'image de $\operatorname{det}_{\mathbb{Z}_{p}\left[G_{n}\right]} \mathcal{D}_{M}(V)_{\Gamma_{n}}^{\Delta_{0}=0} \otimes \operatorname{det}_{\mathbb{Z}_{p}\left[G_{n}\right]}^{-1} \mathscr{D}_{M}(V)_{\Gamma_{n}}$ dans $\mathbb{Q}_{p}\left[G_{n}\right]$ caractère par caractère et d'utiliser la proposition 4.11 .

Si $\eta \in X\left(\Gamma_{1}\right)$ est un caractère non trivial, alors on a $\mathscr{D}(V)_{\eta}^{\Delta_{0}=0} \simeq \mathscr{D}(V)_{\eta}^{\Delta_{0}=0}$ et la formule $e_{\eta} \lambda=\left(\eta\left(\gamma_{1}\right)-1\right) e_{\eta}$ montre que l'image $\operatorname{de} \operatorname{det} \mathscr{D}_{M}(V)_{\eta}^{\Delta_{0}=0} \otimes \operatorname{det}^{-1} \mathscr{D}_{M}(V)_{\eta}$ est engendrée par $\left(\eta\left(\gamma_{1}\right)-1\right)^{\operatorname{dim} \boldsymbol{D}_{\text {cris }}(V)^{\varphi=1}} e_{\eta}$.

Supposons maintenant que $\eta=\eta_{0}$. Dans la preuve de la proposition 4.11, on a vu que la suite exacte

$$
0 \rightarrow \frac{\boldsymbol{D}_{\text {cris }}(V)}{(1-\varphi) \boldsymbol{D}_{\text {cris }}(V)} \rightarrow \mathscr{D}(V)_{\eta_{0}}^{\Delta_{0}=0} \rightarrow \mathscr{D}(V)_{\eta_{0}} \rightarrow \frac{\boldsymbol{D}_{\text {cris }}(V)}{(1-\varphi) \boldsymbol{D}_{\text {cris }}(V)} \rightarrow 0
$$

est isomorphe à la suite :

$$
0 \rightarrow D^{\prime} \rightarrow(1-\varphi) \boldsymbol{D}_{\text {cris }}(V) \oplus D^{\prime} \rightarrow(1-\varphi) \boldsymbol{D}_{\text {cris }}(V) \oplus D^{\prime} \rightarrow D^{\prime} \rightarrow 0,
$$

dont les fleches sont données par $d^{\prime} \mapsto\left(0, p^{n-1} d^{\prime}\right),(a, b) \mapsto(a, 0)$ et $(x, y) \mapsto y$ (voir (4.6)). On en déduit que l'image de $\mathscr{D}_{M}(V)_{\eta_{0}}^{\Delta_{0}=0} \otimes \operatorname{det}^{-1} \mathscr{D}_{M}(V)_{\eta_{0}}$ est engendrée $\operatorname{par} p^{(1-n) \operatorname{dim}_{\mathbb{Q}_{p}} \boldsymbol{D}_{\text {cris }}(V)^{\varphi=1}}$, d'où la proposition. 
Remarque 4.13. On peut remarquer que les facteurs qui sortent dans cette preuve (par exemple $p^{(1-n) \operatorname{dim}_{\mathbb{Q}_{p}} D_{\text {cris }}(V)^{\varphi=1}}$ pour le caractère trivial) compensent des facteurs qui apparaissent dans la preuve de la proposition 4.11 (voir (4.9)) et qu'on n'a pas besoin, donc, de les expliciter pour démontrer la proposition 4.12 .

4.3. Équivalence de $C_{\mathrm{Iw}}$ et de $C_{\mathrm{EP}}$ : étude de $\operatorname{Exp}_{V, \boldsymbol{h}, \boldsymbol{n}}^{\varepsilon}$. Nous passons maintenant à l'étude de l'application :

$$
\operatorname{Exp}_{V, h, n}^{\varepsilon}:\left(D(V)^{\Delta=0}\right)_{\Gamma_{n}} \rightarrow \mathbb{Q}_{p} \otimes_{\mathbb{Z}_{p}}\left(H_{\mathrm{Iw}}^{1}(K, T) / T^{H_{K}}\right)_{\Gamma_{n}}
$$

déduite de $\operatorname{Exp}_{V, h}^{\varepsilon}$. On suppose partout que $h \geqslant 1$ est un entier tel que $\mathrm{Fil}^{-h} \boldsymbol{D}_{\text {cris }}(V)=$ $\boldsymbol{D}_{\text {cris }}(V)$.

Lemme 4.14. Si $V$ est une représentation cristalline, alors :

(1) L'application naturelle de $V^{G_{K}}$ dans $H^{1}\left(\Gamma_{n}, V^{H_{K}}\right)$ est un isomorphisme;

(2) L'application composée :

$$
V^{G_{K}} \rightarrow H^{1}\left(K_{n}, V\right) \stackrel{\exp _{V, K_{n}}^{*}}{\longrightarrow} D_{\mathrm{dR}}^{K_{n}}(V)
$$

cö̈ncide avec l'injection $V^{G_{K}} \stackrel{\log ^{-1} \chi\left(\gamma_{n}\right)}{\longrightarrow} \operatorname{Fil}^{0} D_{\mathrm{dR}}^{K_{n}}(V)$;

(3) On a $V^{G_{K}} \cap H_{g}^{1}\left(K_{n}, V\right)=\{0\}$.

Preuve. Comme $V$ est cristalline, on a un isomorphisme $V^{H_{K}} \simeq \bigoplus_{i \in \mathbb{Z}} \mathbb{Q}_{p}(i)^{d_{i}}$ (voir [Per94, lemme 3.4.3]). La première assertion s'en déduit.

Pour montrer la deuxième, on remarque que l'application $\cup \log \chi: \operatorname{Fil}^{0} D_{\mathrm{dR}}^{K_{n}}(V) \rightarrow$ $H^{1}\left(K_{n}, \mathrm{Fil}^{0} \boldsymbol{B}_{\mathrm{dR}} \otimes V\right)$ est un isomorphisme et que $\exp _{V, K_{n}}^{*}$ coïncide avec l'application composée :

$$
H^{1}\left(K_{n}, V\right) \rightarrow H^{1}\left(K_{n}, \mathrm{Fil}^{0} \boldsymbol{B}_{\mathrm{dR}} \otimes V\right) \stackrel{\sim}{\rightarrow} \mathrm{Fil}^{0} \boldsymbol{D}_{\mathrm{dR}}^{K_{n}}(V)
$$

(c'est la formule de Kato, voir [Kat93a, §1.2-1.4]).

Enfin, comme ker $\left(\exp _{V, K_{n}}^{*}\right)=H_{g}^{1}\left(K_{n}, V\right)$, on en déduit le (3).

Considérons le diagramme commutatif :

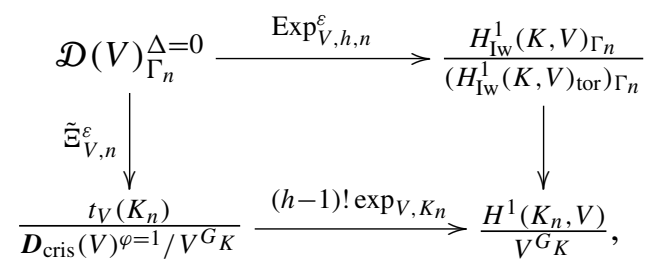


où $H_{\mathrm{Iw}}^{1}(K, V)=H_{\mathrm{Iw}}^{1}(K, T) \otimes_{\mathbb{Z}_{p}} \mathbb{Q}_{p}$ et $H_{\mathrm{Iw}}^{1}(K, V)_{\text {tor }} \simeq V^{H_{K}}$. Par la proposition 2.8, la flèche de droite est injective. Comme la flèche inférieure est injective par définition de l'application exponentielle, on a un isomorphisme :

$$
\operatorname{ker}\left(\operatorname{Exp}_{V, h, n}^{\varepsilon}\right) \simeq \operatorname{ker}\left(\tilde{\Xi}_{V, n}^{\varepsilon}: \mathscr{D}(V)_{\Gamma_{n}}^{\Delta=0} \rightarrow \frac{t_{V}\left(K_{n}\right)}{D_{\text {cris }}(V)^{\varphi=1} / V^{G_{K}}}\right)
$$

La deuxième suite exacte du lemme 1.5 donne un isomorphisme :

$$
\frac{\boldsymbol{D}_{\text {cris }}(V)}{\operatorname{Fil}^{0} \boldsymbol{D}_{\text {cris }}(V)+\left(1-p^{-1} \varphi^{-1}\right) \boldsymbol{D}_{\text {cris }}(V)} \simeq\left(V^{*}(1)^{G_{K}}\right)^{*}
$$

et en quotientant l'isomorphisme (2) de la proposition 3.2 par Fil $^{0}$, on obtient un isomorphisme :

$$
\operatorname{coker}\left(\tilde{\Xi}_{V, n}^{\varepsilon}: \mathcal{D}(V) \Gamma_{\Gamma_{n}}^{\Delta=0} \rightarrow \frac{t_{V}\left(K_{n}\right)}{\boldsymbol{D}_{\text {cris }}(V)^{\varphi=1} / V^{G_{K}}}\right) \stackrel{\operatorname{Tr}_{K_{n} / K}}{\simeq}\left(V^{*}(1)^{G_{K}}\right)^{*}
$$

d'où la suite exacte courte :

$$
0 \rightarrow \frac{\mathscr{D}(V)_{\Gamma_{n}}^{\Delta=0}}{\operatorname{ker}\left(\operatorname{Exp}_{V, h, n}^{\varepsilon}\right)} \stackrel{\tilde{\Xi}_{V, n}^{\varepsilon}}{\longrightarrow} \frac{t_{V}\left(K_{n}\right)}{\boldsymbol{D}_{\text {cris }}(V)^{\varphi=1} / V^{G_{K}}} \stackrel{\operatorname{Tr}_{K_{n} / K}}{\longrightarrow}\left(V^{*}(1)^{G_{K}}\right)^{*} \rightarrow 0
$$

D'autre part, pour toute représentation $p$-adique, on a une suite exacte :

$$
0 \rightarrow\left(\frac{H_{\mathrm{Iw}}^{1}(K, V)}{V^{H_{K}}}\right)_{\Gamma_{n}} \rightarrow \frac{H^{1}\left(K_{n}, V\right)}{H^{1}\left(\Gamma_{n}, V^{H_{K}}\right)} \rightarrow H_{\mathrm{IW}}^{2}(K, V)^{\Gamma_{n}} \rightarrow 0
$$

(voir la proposition 2.8 ou bien [Per94, proposition 3.2.1]). On a

$$
V^{G_{K}} \simeq H^{1}\left(\Gamma_{n}, V^{H_{K}}\right) \quad \text { et } \quad H_{\mathrm{Iw}}^{2}(K, V)^{\Gamma_{n}} \simeq\left(\left(V^{*}(1)^{H_{K}}\right)^{*}\right)^{\Gamma_{n}} \simeq\left(V^{*}(1)^{G_{K}}\right)^{*} .
$$

La flèche $H^{1}\left(K_{n}, V\right) \rightarrow H_{\mathrm{IW}}^{2}(K, V)^{\Gamma_{n}}$ est duale de l'application d'inflation $V^{*}(1)^{G_{K}} \rightarrow H^{1}\left(K_{n}, V^{*}(1)\right)$ (voir le (3) de la remarque 2.9).

Proposition 4.15. On a un diagramme commutatif dont les flèches horizontales sont 
des isomorphismes :

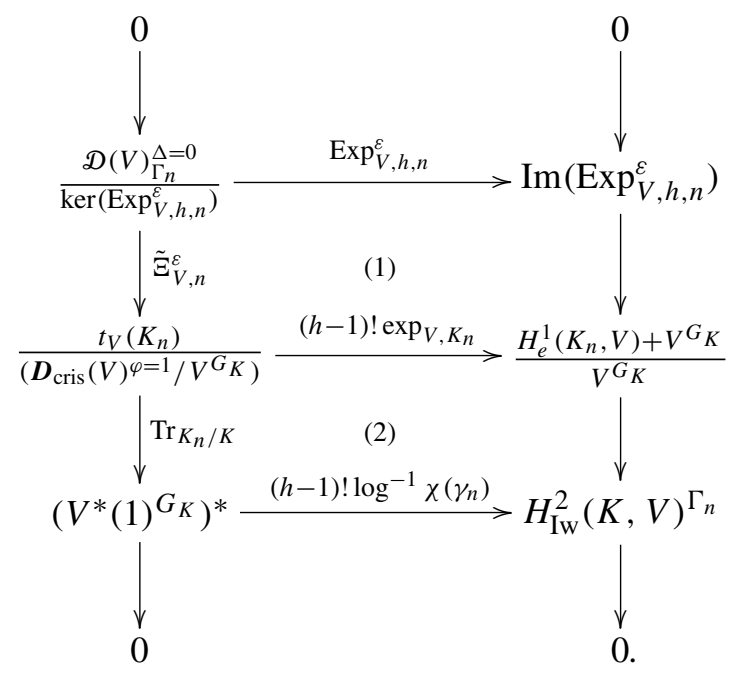

Preuve. Il résulte du théorème 3.1 que le carré (1) du diagramme est commutatif. D'après le lemme 4.14, le diagramme :

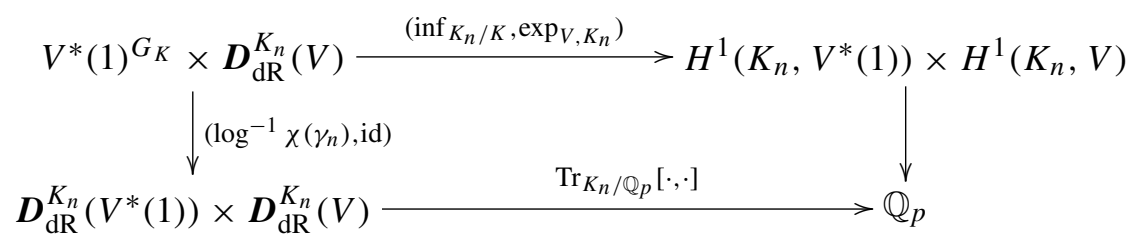

est commutatif, ce qui fait que l'application composée :

$$
D_{\mathrm{dR}}^{K_{n}}(V) \stackrel{\exp _{V, K_{n}}}{\longrightarrow} H^{1}\left(K_{n}, V\right) \rightarrow\left(V^{*}(1)^{G_{K}}\right)^{*}
$$

coïncide avec l'application :

$$
\boldsymbol{D}_{\mathrm{dR}}^{K_{n}}(V) \stackrel{\log ^{-1} \chi\left(\gamma_{n}\right) \operatorname{Tr}_{K_{n} / K}}{\longrightarrow} \boldsymbol{D}_{\text {cris }}(V) \rightarrow\left(V^{*}(1)^{G_{K}}\right)^{*} .
$$

On en déduit que le carré (2) du diagramme commute. Il est clair que toutes les flèches horizontales sont des isomorphismes. Comme la colonne de gauche est exacte, la colonne de droite l'est aussi.

Corollaire 4.16. On a un isomorphisme canonique :

$$
\operatorname{coker}\left(\operatorname{Exp}_{V, h, n}^{\varepsilon}\right) \simeq \frac{H^{1}\left(K_{n}, V\right)}{H_{e}^{1}\left(K_{n}, V\right)+V^{G_{K}}} .
$$


Preuve. Il suffit d'appliquer le lemme du serpent au diagramme :

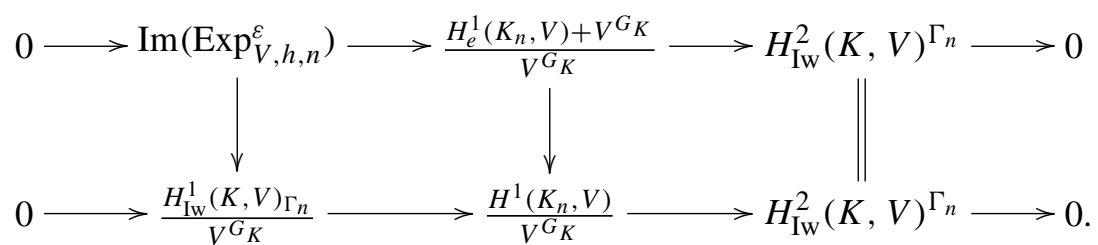

On note $B_{V, h, n}^{\varepsilon}: \operatorname{ker}\left(\operatorname{Exp}_{V, h, n}^{\varepsilon}\right) \rightarrow \operatorname{coker}\left(\operatorname{Exp}_{V, h, n}^{\varepsilon}\right)$ l'application déduite de $\operatorname{Exp}_{V, h, n}^{\varepsilon}$.

Proposition 4.17. On a un diagramme commutatif dont les flèches horizontales sont des isomorphismes :

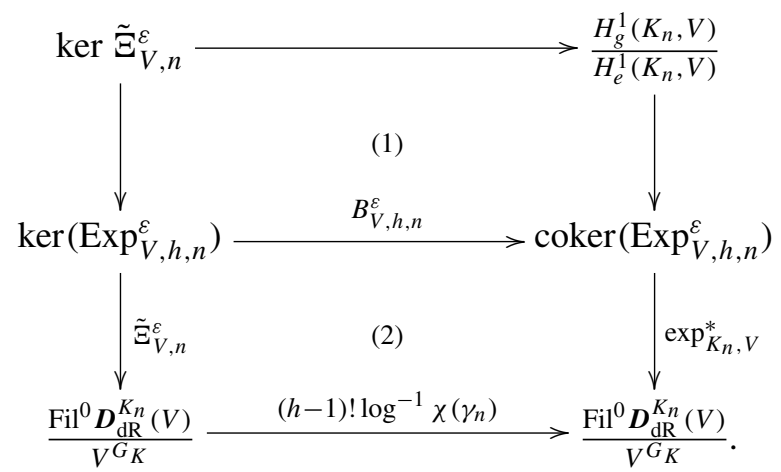

Preuve. La commutativité du deuxième carré est démontrée dans [Per94, lemme 3.5.9] en utilisant la loi de réciprocité explicite. Remarquons que Perrin-Riou utilise une autre normalisation pour la flèche au milieu ( $\operatorname{son} t_{\gamma}$ est égal à notre $-B_{V, h, n}^{\varepsilon}$ ) ce qui fait apparaître le signe dans sa formule. Le reste est une conséquence immédiate de la proposition 4.18 ci-dessous.

Rappelons que dans le lemme 1.3, on a construit des isomorphismes :

$$
\begin{aligned}
& \exp _{V, f / e}: \frac{\boldsymbol{D}_{\text {cris }}(V)}{(1-\varphi) \boldsymbol{D}_{\text {cris }}(V)} \stackrel{\sim}{\rightarrow} \frac{H_{f}^{1}\left(K_{n}, V\right)}{H_{e}^{1}\left(K_{n}, V\right)}, \\
& \exp _{V, g / f}^{*}: \frac{H_{g}^{1}\left(K_{n}, V\right)}{H_{f}^{1}\left(K_{n}, V\right)} \stackrel{\sim}{\rightarrow} \boldsymbol{D}_{\text {cris }}(V)^{\varphi=p^{-1}} .
\end{aligned}
$$


Proposition 4.18. Le diagramme :

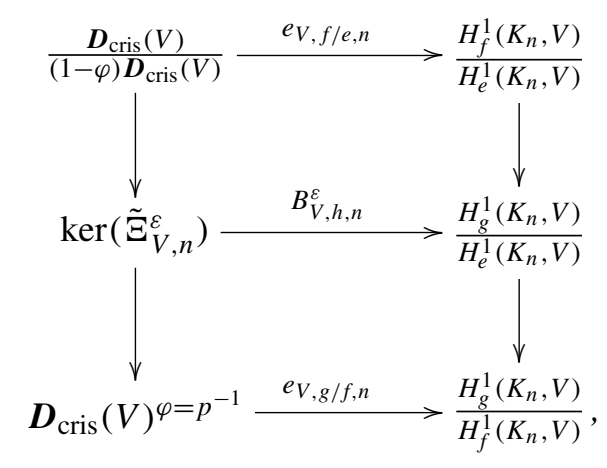

où

$$
\begin{aligned}
& e_{V, f / e, n}(a)=(h-1) ! p^{-n} \exp _{V, f / e}(a) \\
& e_{V, g / f, n}(b)=\left(1-\frac{1}{p}\right)^{-1}(h-1) ! \log ^{-1} \chi\left(\gamma_{n}\right)\left(\exp _{V, g / f}^{*}\right)^{-1}(b),
\end{aligned}
$$

est commutatif et où la colonne de gauche est donnée par le (1) de la proposition 3.2.

Preuve. Si $a \in \boldsymbol{D}_{\text {cris }}(V) /(1-\varphi) \boldsymbol{D}_{\text {cris }}(V)$, on choisit un élément $f(X) \in \mathscr{D}(V)$ vérifiant $\Delta f(X)=a$ et l'on pose $g(X)=\left(\gamma_{n}-1\right) f(X)$ ce qui fait que $g(X)$ est l'image de $a$ dans $\operatorname{ker}\left(\tilde{\Xi}_{V, n}^{\varepsilon}\right) \subset \mathscr{D}(V)_{\Gamma_{n}}^{\Delta=0}$. Soit $F(X) \in \mathscr{H}(V)$ un élément vérifiant l'équation $(1-\varphi) F(X)=f(X)-a$. Si on pose $\alpha(X)=\left(\partial^{h} \otimes e_{h}\right) f(X)$ et $A(X)=\left(\partial^{h} \otimes e_{h}\right) F(X)$, alors $A(X)$ vérifie $(1-\varphi) A(X)=\alpha(X)$.

On a $\mathrm{Fil}^{0} \boldsymbol{D}_{\text {cris }}(V(-h))=\boldsymbol{D}_{\text {cris }}(V(-h))$; soit :

$$
\Sigma_{V(-h), h+k, m}^{\varepsilon}: \mathscr{H}(V(-h)) \rightarrow H^{1}\left(K_{m}, V(k)\right)
$$

le système d'applications construit dans [Ben00, §4.2-4.3] et dont la construction a été rappelée au paragraphe 3.2. Posons :

$$
z_{k, m}=(-1)^{h} \Sigma_{V(-h), h+k, m}^{\varepsilon}\left((\sigma \otimes \varphi)^{-m} A(X)\right),
$$

et notons $\bar{z}_{k, m}$ son image dans $H^{1}\left(K_{m}, V(k)\right) / H^{1}\left(\Gamma_{m}, V(k)^{H_{K}}\right)$. Le théorème $4.3 \mathrm{de}$ [Ben00] montre que $\operatorname{cor}_{K_{n+1} / K_{n}}\left(\bar{z}_{k, n+1}\right)=\bar{z}_{k, n}$ pour tout $n \geqslant 1$ et qu'il existe $s \geqslant 0$ tel que la suite :

$$
p^{(s-j) m} \sum_{k=0}^{j}(-1)^{k}\left(\begin{array}{l}
j \\
k
\end{array}\right) \operatorname{Tw}_{-k}^{\varepsilon} \circ \operatorname{res}_{K_{\infty} / K_{n}}\left(z_{m, k}\right)
$$


converge vers 0 quand $m \rightarrow \infty$. On vérifie que $\bar{z}_{k, m} \in\left(H_{\mathrm{Iw}}^{1}(K, V(k)) / V(k)^{H_{K}}\right)_{\Gamma_{m}}$ (par exemple, on peut utiliser les arguments de [Ben00], pour montrer que le cupproduit de $\bar{z}_{k, m}$ avec les éléments de $V^{*}(1-k)^{G_{K}}$ est nul) et il existe donc un unique élément $z \in \mathcal{H}(\Gamma) \otimes_{\Lambda}\left(H_{\mathrm{IW}}^{1}(K, V(k)) / V(k)^{H_{K}}\right)$ tel que $\operatorname{pr}_{V(k), m}\left(\operatorname{Tw}_{k}^{\varepsilon}(z)\right)=\bar{z}_{k, m}$ pour tous $k \in \mathbb{Z}$ et $m \geqslant 1$.

L'élément $B(X)=\left(\gamma_{n}-1\right) A(X)$ vérifie $(1-\varphi) B(X)=\left(\partial^{h} \otimes e_{h}\right) g(X)$ et on a :

$$
\operatorname{pr}_{V(k), m}\left(\operatorname{Tw}_{V, k}^{\varepsilon} \circ \operatorname{Exp}_{V, h}^{\varepsilon}(g)\right)=(-1)^{h} \Sigma_{V(-h), h+k, m}^{\varepsilon}\left((\sigma \otimes \varphi)^{-m} B(X)\right),
$$

et donc $\left(\gamma_{n}-1\right) z=\operatorname{Exp}_{V, h}^{\varepsilon}(g)$ et $B_{V, h, n}^{\varepsilon}(a)=-z_{0, n} \bmod H_{e}^{1}\left(K_{n}, V\right)$. D'autre part, le même argument que dans [Ben00, §4.4.5] montre que :

$$
z_{0, n}=(h-1) ! p^{-n} \exp _{V, K_{n}}\left(-\varphi^{-n}(a),(\sigma \otimes \varphi)^{-n} F\left(\zeta_{p^{n}}-1\right)\right),
$$

où on note encore $\exp _{V, K_{n}}$ l'application de connexion dans (1.2) :

$$
\exp _{V, K_{n}}: \boldsymbol{D}_{\text {cris }}(V) \oplus t_{V}\left(K_{n}\right) \rightarrow H^{1}\left(K_{n}, V\right) .
$$

Comme l'opérateur $\varphi$ agit trivialement $\operatorname{sur} \boldsymbol{D}_{\text {cris }}(V) /(1-\varphi) \boldsymbol{D}_{\text {cris }}(V)$, on en déduit que $(h-1) ! p^{-n} \exp _{V, f / e}(a)=-z_{0, n} \bmod H_{e}^{1}\left(K_{n}, V\right)$, d'où on obtient $B_{V, h, n}^{\varepsilon}(a)=$ $(h-1) ! p^{-n} \exp _{V, f / e}(a)$ ce qui montre la commutativité du premier carré du diagramme.

Démontrons la commutativité du deuxième carré. Fixons un entier $k \geqslant 1$ supérieur à la longueur de la filtration de Hodge de $V^{*}(1)$ et tel que Fil ${ }^{-k} \boldsymbol{D}_{\text {cris }}\left(V^{*}(1)\right)=$ $\boldsymbol{D}_{\text {cris }}\left(V^{*}(1)\right)$. Comme $\operatorname{Exp}_{V^{*}(1), j}^{\varepsilon^{-1}}=\ell_{j-1} \operatorname{Exp}_{V^{*}(1), j-1}^{\varepsilon^{-1}}$ et $\ell_{j}^{l}=-\ell_{-j}$, la loi de réciprocité s'écrit :

$$
\left\langle\operatorname{Exp}_{V, h}^{\varepsilon}(f), \operatorname{Exp}_{V^{*}(1), k}^{\varepsilon^{-1}}\left(g^{\iota}\right)\right\rangle(1+X)=\left(\prod_{i=1}^{h-1} \ell_{i}\right)\left(\prod_{j=1}^{k-1} \ell_{j}^{\iota}\right) \operatorname{Tr}_{K / \mathbb{Q}_{p}}(f \star \mathbb{D} g) .
$$

Si $f \in \operatorname{ker}\left(\tilde{\Xi}_{V, n}^{\varepsilon}\right)$, alors $\operatorname{Exp}_{V, h}^{\varepsilon}(f)=\left(\gamma_{n}-1\right) x$ où $x \in \mathcal{H}(\Gamma) \otimes H_{\mathrm{IW}}^{1}(K, V)$ et $B_{V, h, n}^{\varepsilon}(f)=-\operatorname{pr}_{V, n}(x) \bmod H_{e}^{1}\left(K_{n}, V\right)$.

Soient $b \in \boldsymbol{D}_{\text {cris }}\left(V^{*}(1)\right) /(1-\varphi) \boldsymbol{D}_{\text {cris }}\left(V^{*}(1)\right)$ et $\beta(X) \in \mathscr{D}\left(V^{*}(1)\right)$ un élément vérifiant $\Delta \beta(X)=b$. Posons $g(X)=\left(\gamma_{n}-1\right) \beta(X)$. On a alors :

$$
\begin{aligned}
\operatorname{Exp}_{V^{*}(1), k}^{\varepsilon^{-1}}(g) & =\left(\gamma_{n}-1\right) y, \\
\left\langle\operatorname{Exp}_{V, h}^{\varepsilon}(f), \operatorname{Exp}_{V^{*}(1), k}^{\varepsilon^{-1}}\left(g^{l}\right)\right\rangle & =\left(\gamma_{n}-1\right)^{2}\left\langle x, y^{\iota}\right\rangle .
\end{aligned}
$$

D'autre part, par la loi de réciprocité explicite, on a :

$$
\begin{aligned}
& \left\langle\operatorname{Exp}_{V, h}^{\varepsilon}(f), \operatorname{Exp}_{V^{*}(1), k}^{\varepsilon^{-1}}\left(g^{\iota}\right)\right\rangle(1+X) \\
& \quad=\left(\gamma_{n}-1\right)^{2}\left(\prod_{i=1}^{h-1} \ell_{i}\right)\left(\prod_{j=1}^{k-1} \ell_{j}^{\iota}\right) \frac{\ell_{0}^{\iota}}{\gamma_{n}-1} \operatorname{Tr}_{K / \mathbb{Q}_{p}}\left(f \star_{D} \beta\right),
\end{aligned}
$$


d'où :

$$
\left\langle x, y^{\iota}\right\rangle(1+X)=\frac{\ell_{0}^{\iota}}{\gamma_{n}-1}\left(\prod_{i=1}^{h-1} \ell_{i}\right)\left(\prod_{j=1}^{k-1} \ell_{j}^{\iota}\right) \operatorname{Tr}_{K / \mathbb{Q}_{p}}\left(f \star_{D} \beta\right) .
$$

On a $\operatorname{pr}_{V^{*}(1), n}(y) \in H_{f}^{1}\left(K_{n}, V^{*}(1)\right)$ et $B_{V^{*}(1), k, n}^{\varepsilon}(b)$ est égale à $-\operatorname{pr}_{V^{*}(1), n}(y)$ modulo $H_{e}^{1}\left(K_{n}, V^{*}(1)\right)$. Il est facile de voir que $g^{l}$ vérifie aussi $\Delta\left(g^{l}\right)=b$ et que $B_{V, k, n}^{\varepsilon}(b)$ ne dépend pas du choix de $\varepsilon$ d'où :

$$
B_{V^{*}(1), k, n}^{\varepsilon}(b)=-\operatorname{pr}_{V^{*}(1), n}\left(y^{\iota}\right) \bmod H_{e}^{1}\left(K_{n}, V^{*}(1)\right) .
$$

Comme $\ell_{0} \equiv-\frac{\gamma_{n}-1}{\log \chi\left(\gamma_{n}\right)} \bmod \left(\gamma_{n}-1\right)^{2}$ et comme le coefficient de $(1+X)$ dans le polynôme d'interpolation de $\operatorname{Tr}_{K / \mathbb{Q}_{p}}\left(f \star_{D} \beta\right)$ modulo $(1+X)^{p^{n}}-1$ est égal à :

$$
\frac{1}{p^{n}} \operatorname{Tr}_{K / \mathbb{Q}_{p}} \sum_{\zeta \in \mu_{p^{n}}}\left[f(\zeta-1), \beta^{\iota}\left(\zeta^{-1}-1\right)\right]_{V}
$$

(voir, par exemple, [Per94, proposition 4.3.2]), on déduit de la loi de réciprocité la formule suivante :

$$
\begin{aligned}
& \left(B_{V, h, n}^{\varepsilon}(f), B_{V^{*}(1), k, n}^{\varepsilon}(b)\right)_{V, K_{n}} \\
& \quad=\frac{(h-1) !(k-1) !}{p^{n} \log \chi\left(\gamma_{n}\right)} \operatorname{Tr}_{K / \mathbb{Q}_{p}} \sum_{\zeta \in \mu_{p^{n}}}\left[f(\zeta-1), \beta^{\iota}\left(\zeta^{-1}-1\right)\right]_{V} .
\end{aligned}
$$

Soit $F(X)$ un élément tel que $(1-\varphi) F(X)=f(X)$. Comme $\tilde{\Xi}_{V, n}^{\varepsilon}(f)=0$, on a $F\left(\zeta_{p^{n}}-1\right) \in \boldsymbol{D}_{\text {cris }}(V)^{\varphi=1}$. On peut modifier $F(X)$ par cet élément et on a alors $F\left(\zeta_{p^{n}}-1\right)=0$. Comme $F(X)$ vérifie l'équation $\sum_{\zeta^{p}=1} F(\zeta(1+X)-1)=p F^{\varphi}(X)$, on a $F\left(\zeta_{p^{m}}-1\right)=0$ pour tout $1 \leqslant m \leqslant n$. On en déduit que $f\left(\zeta_{p^{m}}-1\right)=0$ si $2 \leqslant m \leqslant n$ et $f\left(\zeta_{p}-1\right)=-\varphi(F(0))$. D'autre part, on a $(1-\varphi) F(0)=$ $f(0)$ et comme $f(0) \in \boldsymbol{D}_{\text {cris }}(V)^{\varphi=p^{-1}}$, on en déduit que $F(0)=(1-1 / p)^{-1} f(0)$ $\bmod \boldsymbol{D}_{\text {cris }}(V)^{\varphi=1}$. Comme $\sum_{\zeta \in \mu_{p}} \beta^{\iota}\left(\zeta^{-1}-1\right)=0$, on obtient :

$$
\begin{aligned}
\sum_{\zeta \in \mu_{p^{n}}} & {\left[f(\zeta-1), \beta^{l}\left(\zeta^{-1}-1\right)\right]_{V} } \\
= & \sum_{\zeta \in \mu_{p}}\left[f(\zeta-1), \beta^{\iota}\left(\zeta^{-1}-1\right)\right]_{V} \\
= & \sum_{\zeta \in \mu_{p}}\left[-\varphi(F(0)), \beta^{l}\left(\zeta^{-1}-1\right)\right]_{V}+[f(0)+\varphi(F(0)), \beta(0)]_{V} \\
= & {[F(0), b]_{V} } \\
= & \left(1-\frac{1}{p}\right)^{-1}[f(0), b]_{V},
\end{aligned}
$$


d'où :

$$
\left(B_{V, h, n}^{\varepsilon}(f), B_{V^{*}(1), k, n}^{\varepsilon}(b)\right)_{V, K_{n}}=\left(1-\frac{1}{p}\right)^{-1} \frac{(h-1) !(k-1) !}{p^{n} \log \chi\left(\gamma_{n}\right)} \operatorname{Tr}_{K / \mathbb{Q}_{p}}[f(0), b]_{V} .
$$

Comme $B_{V^{*}(1), h, n}^{\varepsilon}(b)=e_{V^{*}(1), f / e, n}(b)$, la commutativité du deuxième carré résulte de la définition de l'application $\exp _{V, g / f}^{*}$.

Preuve du théorème 4.4. Soit $h \geqslant 1$ un entier vérifiant $\operatorname{Fil}^{-h} \boldsymbol{D}_{\text {cris }}(V)=\boldsymbol{D}_{\text {cris }}(V)$ et soit :

$$
\delta_{V, K_{\infty} / K, h}^{\prime}: \Delta_{\mathrm{PR}}\left(K_{\infty} / K, V\right) \rightarrow \mathscr{H}(\Gamma)
$$

la trivialisation de la droite :

$$
\Delta_{\mathrm{PR}}\left(K_{\infty} / K, V\right) \simeq \otimes_{i=1}^{2}\left(\operatorname{det}_{\Lambda_{\mathbb{Q}_{p}}} H_{\mathrm{IW}}^{i}(K, V)\right)^{(-1)^{i}} \otimes_{\Lambda_{\mathbb{Q}_{p}}} \operatorname{det}_{\Lambda_{\mathbb{Q}_{p}}} \mathcal{D}(V)
$$

qui a été construite dans le paragraphe 4.1.

Soit $n \geqslant 1$. Comme $\ell_{0}=\log \gamma_{n} / \log \chi\left(\gamma_{n}\right) \equiv \frac{\gamma_{n}-1}{\log \chi\left(\gamma_{n}\right)} \bmod \left(\gamma_{n}-1\right)^{2}$, il est une conséquence du théorème 4.2 que l'image de $\delta_{V, K_{\infty} / K, h}^{\prime}$ est contenue dans $\left(1-\gamma_{n}\right)^{\operatorname{dim}_{\mathbb{Q}_{p}} \operatorname{Fil}^{0} \boldsymbol{D}_{\text {cris }}(V)} \mathcal{H}(\Gamma)$ et on pose :

$$
\delta_{V, K_{\infty} / K, h}^{*}=\left(1-\gamma_{n}\right)^{-\operatorname{dim}_{\mathbb{Q}_{p}} \operatorname{Fil}^{0} \boldsymbol{D}_{\text {cris }}(V)} \delta_{V, K_{\infty} / K, h}
$$

Soit $\mathbb{Q}_{p}\left[G_{n}\right] \simeq \bigoplus_{\lambda} E_{\lambda}$ la décomposition de $\mathbb{Q}_{p}\left[G_{n}\right]$ en somme directe de corps. Par la proposition 4.6, l'application $\operatorname{Exp}_{V, h}^{\varepsilon}$ est admissible et le lemme 4.5 fournit un diagramme commutatif :

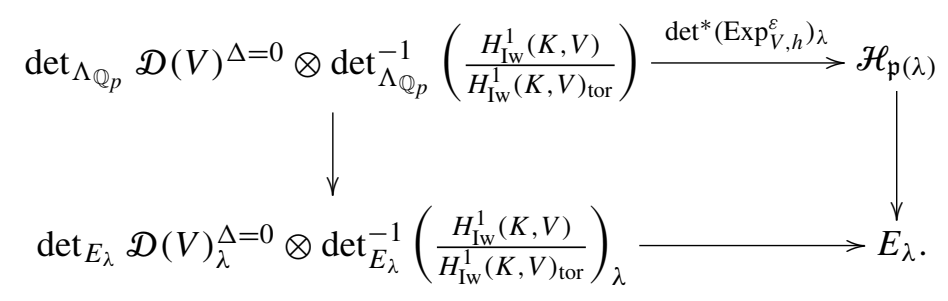

La flèche inférieure de ce diagramme se décompose en produit des déterminants des applications :

$$
\begin{aligned}
\operatorname{Exp}_{V, h, \lambda}^{\varepsilon}: \frac{\mathscr{D}(V)_{\lambda}^{\Delta=0}}{\operatorname{ker}\left(\operatorname{Exp}_{V, h, \lambda}^{\varepsilon}\right)} \stackrel{\sim}{\rightarrow} \operatorname{Im}\left(\operatorname{Exp}_{V, h, \lambda}^{\varepsilon}\right) \\
B_{V, h, \lambda}^{\varepsilon}: \operatorname{ker}\left(\operatorname{Exp}_{V, h, \lambda}^{\varepsilon}\right) \stackrel{\sim}{\rightarrow} \operatorname{coker}\left(\operatorname{Exp}_{V, h, \lambda}^{\varepsilon}\right) .
\end{aligned}
$$


Comme $H_{\mathrm{Iw}}^{2}(K, V) \simeq\left(V^{*}(1)^{H_{K}}\right)^{*}$ est admissible et comme $H_{\mathrm{IW}}^{2}(K, V)_{\Gamma_{n}} \simeq$ $H^{2}\left(K_{n}, V\right)$, le lemme 4.5 donne un diagramme :

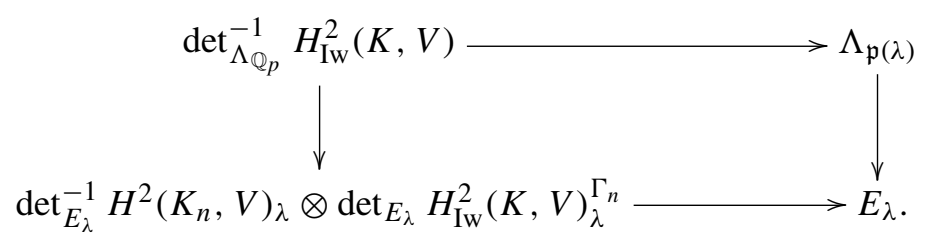

Il est clair que $H^{2}\left(K_{n}, V\right)_{\lambda}=H_{\mathrm{Iw}}^{2}(K, V)_{\lambda}^{\Gamma_{n}}=0$ si $\lambda \neq \lambda_{0}$ et que pour $\lambda=\lambda_{0}$, la flèche inférieure de (4.17) s'identifie avec le déterminant de l'application identité : $\left(V^{*}(1)^{G_{K}}\right)^{*} \stackrel{\text { id }}{\rightarrow}\left(V^{*}(1)^{G_{K}}\right)^{*}$. On a des diagrammes du même type pour les modules $\mathscr{D}(V) / \mathscr{D}(V)^{\Delta=0}$ et $H_{\mathrm{Iw}}^{1}(K, V)_{\mathrm{tor}} \simeq V^{H_{K}}$. En particulier, comme $H_{\mathrm{Iw}}^{1}(K, V)_{\text {tor }}^{\Gamma_{n}}=$ $H_{\mathrm{IW}}^{1}(K, V)^{\Gamma_{n}} \simeq H^{0}\left(K_{n}, V\right)$, on a un diagramme :

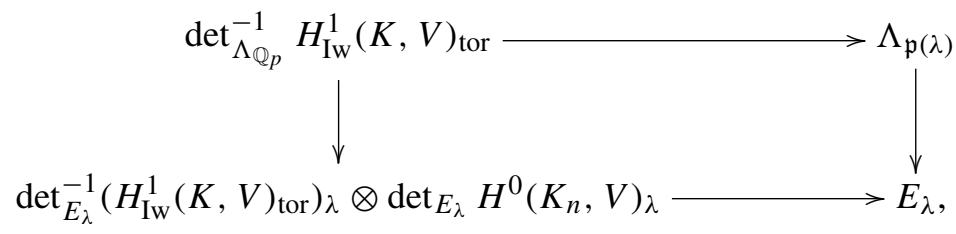

où $H^{0}\left(K_{n}, V\right)_{\lambda}=0$ si $\lambda \neq \lambda_{0}$ et $H^{0}\left(K_{n}, V\right)_{\lambda_{0}}=V^{G_{K}}$.

Il résulte du théorème 4.2 que le produit des flèches supérieures des diagrammes (4.14), (4.17) et (4.18) coïncide avec la localisation de $\delta_{V, K_{\infty} / K, h}^{*}$ en $\mathfrak{p}(\lambda)$. En mettant ces diagrammes ensemble et en utilisant la suite exacte (3) de la proposition 2.8 , on obtient un diagramme commutatif :

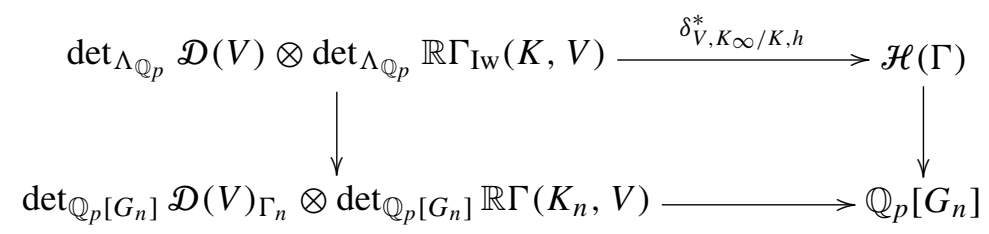

Nous allons identifier la flèche inférieure de ce diagramme avec l'isomorphisme $\delta_{V, K_{n} / K}^{\prime}$ qui a été défini au paragraphe 2.5. En effet, $\delta_{V, K_{n} / K}^{\prime}$ se décompose en le 
produit des déterminants des suites exactes et isomorphismes suivants :

$$
\begin{gathered}
0 \rightarrow H^{0}\left(K_{n}, V\right) \rightarrow \boldsymbol{D}_{\text {cris }}(V)^{\varphi=1} \rightarrow t_{V}\left(K_{n}\right) \rightarrow H_{e}^{1}\left(K_{n}, V\right) \rightarrow 0, \\
\exp _{V, f / e}: \boldsymbol{D}_{\text {cris }}(V) /(1-\varphi) \boldsymbol{D}_{\text {cris }}(V) \stackrel{\sim}{\rightarrow} \frac{H_{f}^{1}\left(K_{n}, V\right)}{H_{e}^{1}\left(K_{n}, V\right)}, \\
\exp _{V, g / f}^{*}: \frac{H_{g}^{1}\left(K_{n}, V\right)}{H_{f}^{1}\left(K_{n}, V\right)} \simeq \boldsymbol{D}_{\text {cris }}(V)^{\varphi=p^{-1}}, \\
0 \rightarrow \frac{H^{1}\left(K_{n}, V\right)}{H_{g}^{1}\left(K_{n}, V\right)} \rightarrow \operatorname{Fil}^{0} \boldsymbol{D}_{\mathrm{dR}}^{K_{n}}(V) \rightarrow \frac{\boldsymbol{D}_{\text {cris }}(V)}{\left(1-p^{-1} \varphi^{-1}\right) \boldsymbol{D}_{\text {cris }}(V)} \rightarrow\left(V^{*}(1)^{G_{K}}\right)^{*} \rightarrow 0
\end{gathered}
$$

(voir (1.1), lemme 1.3 et lemme 1.5).

D'autre part, les propositions 4.15 et 4.17 fournissent un diagramme commutatif (voir figure 1, page suivante) dont les flèches sont données par les formules suivantes :

- (1) est la restriction de $B_{V, h, n}^{\varepsilon}$ à $\operatorname{ker}\left(\tilde{\Xi}_{V, n}^{\varepsilon}\right)$;

- (2) est $\frac{\log \chi\left(\gamma_{n}\right)}{(h-1) !} \exp _{V, K_{n}}^{*}$;

- (3) et (4) coïncident avec les applications correspondantes dans (4.20);

- (5) est $(h-1)$ ! $\exp _{V, K_{n}}$;

- (6) est la multiplication par $(h-1) ! \log ^{-1} \chi\left(\gamma_{n}\right)$ composée avec l'isomorphisme canonique $\left(V^{*}(1)^{G_{K}}\right)^{*} \simeq H_{\mathrm{IW}}^{2}(K, V)^{\Gamma_{n}}$;

- (7) et (8) sont induites par les isomorphismes de projection $H_{\mathrm{Iw}}^{2}(K, V)^{\Gamma_{n}} \rightarrow$ $H_{\mathrm{Iw}}^{2}(K, V)_{\Gamma_{n}}$ et $H_{\mathrm{IW}}^{1}(K, V)^{\Gamma_{n}} \rightarrow\left(H_{\mathrm{Iw}}^{1}(K, V)_{\mathrm{tor}}\right)_{\Gamma_{n}}$.

Sur la deuxième ligne de ce diagramme, on trouve les isomorphismes (4.15) et (4.16). Grâce à la proposition 4.18, les parties encadrées s'identifient, à multiplication des flèches par des constantes explicites près, avec les suites exactes et isomorphismes (4.20). Les flèches en pointillés montrent avec quelles parties de ces suites s'identifient $H_{\mathrm{Iw}}^{2}(K, V)_{\Gamma_{n}}=H^{2}(K, V)$ et $H^{0}(K, V)$. Par fonctorialité des déterminants, on déduit du gros diagramme le diagramme commutatif ci-dessous :

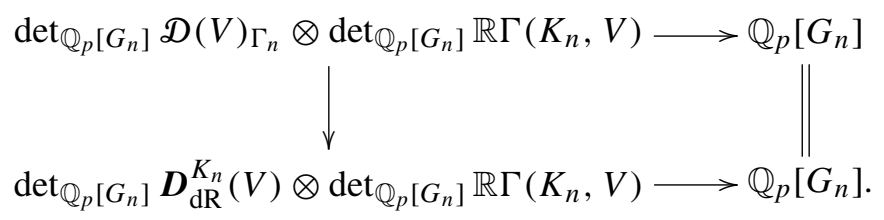

La flèche gauche de (4.21) est induite par $\kappa_{V, n}$ et par l'application identité sur $\operatorname{det}_{\mathbb{Q}_{p}\left[G_{n}\right]} \mathbb{R} \Gamma\left(K_{n}, V\right)$. Par le lemme 4.14, l'application composée :

$$
H^{0}(K, V) \stackrel{(7)}{\longrightarrow}\left(H_{\mathrm{Iw}}^{1}(K, V)_{\mathrm{tor}}\right)_{\Gamma_{n}} \stackrel{(2)}{\longrightarrow} V^{G_{K}}
$$




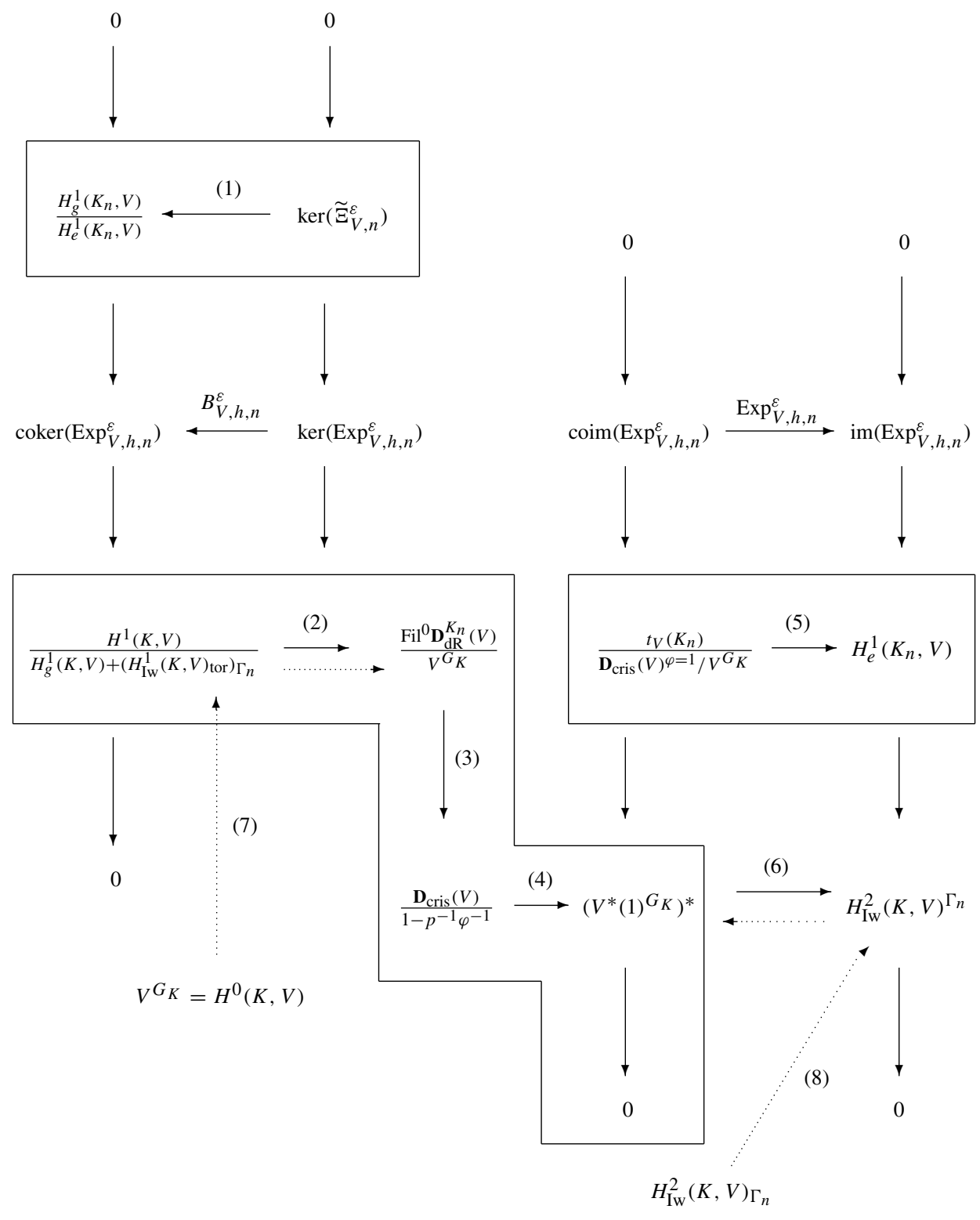

Figure 1 
coïncide avec la multiplication par $((h-1) !)^{-1}$. D'autre part, le composé :

$$
H^{2}\left(K_{n}, V\right) \stackrel{(8)}{\longrightarrow} H_{\mathrm{Iw}}^{2}(K, V)^{\Gamma_{n}} \rightarrow\left(V^{*}(1)^{G_{K}}\right)^{*} \simeq H^{2}\left(K_{n}, V\right)
$$

coïncide avec la multiplication par $\log \chi\left(\gamma_{n}\right) /(h-1)$ !. Un petit calcul utilisant les propositions $4.15,4.17$ et 4.18 montre que la flèche inférieure de (4.21) coïncide avec $\delta_{V, K_{n} / K}^{\prime}$ multiplié par :

$$
\begin{aligned}
&((h-1) !)^{\operatorname{dim}_{\mathbb{Q}_{p}} \boldsymbol{D}_{\text {cris }}(V)}\left(\log \chi\left(\gamma_{n}\right)\right)^{-\operatorname{dim}_{\mathbb{Q}_{p}} \operatorname{Fil}^{0} \boldsymbol{D}_{\text {cris }}(V)} \\
& \cdot\left(1+\left(p^{-n \operatorname{dim}_{\mathbb{Q}_{p}} \boldsymbol{D}_{\text {cris }}(V)^{\varphi=1}}\left(1-\frac{1}{p}\right)^{-\operatorname{dim}_{\mathbb{Q}_{p}} \boldsymbol{D}_{\text {cris }}(V)^{\varphi=p^{-1}}}-1\right) e_{\eta_{0}}\right) .
\end{aligned}
$$

Par le lemme 4.7, on a :

$$
((h-1) !)^{\operatorname{dim}_{\mathbb{Q}_{p}} \boldsymbol{D}_{\text {cris }}(V)}\left(\log \chi\left(\gamma_{n}\right)\right)^{-\operatorname{dim}_{\mathbb{Q}_{p}} \operatorname{Fil}^{0} \boldsymbol{D}_{\text {cris }}(V)}= \pm \boldsymbol{\Gamma}_{h}^{*}(V) \boldsymbol{\Gamma}(V) .
$$

On obtient enfin le diagramme suivant, qui résume la descente effectuée :

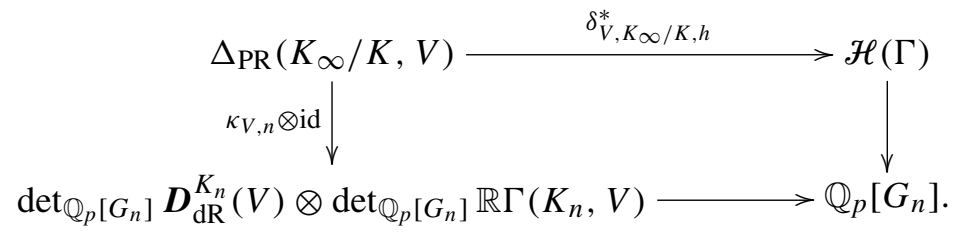

La flèche inférieure de ce diagramme est :

$$
\pm \boldsymbol{\Gamma}_{h}^{*}(V) \boldsymbol{\Gamma}(V)\left(1+\left(p^{-n \operatorname{dim}_{\mathbb{Q}_{p}} \boldsymbol{D}_{\text {cris }}(V)^{\varphi=1}}\left(1-\frac{1}{p}\right)^{-\operatorname{dim}_{\mathbb{Q}_{p}} \boldsymbol{D}_{\text {cris }}(V)^{\varphi=p^{-1}}}-1\right) e_{\eta_{0}}\right) \delta_{V, K_{n} / K}^{\prime}
$$

Nous pouvons maintenant terminer la preuve du théorème 4.4. En effet, par le théorème $4.2, \delta_{V, K_{\infty} / K, h}^{*}$ envoie $\operatorname{det}_{\Lambda} \mathscr{D}_{M}(V) \otimes \operatorname{det}_{\Lambda} \mathbb{R} \Gamma_{\mathrm{Iw}}(K, T)$ sur un $\Lambda$-module libre de la forme :

$$
\left(1-\gamma_{n}\right)^{-\operatorname{dim}_{\mathbb{Q}_{p}} \operatorname{Fil}^{0} \boldsymbol{D}_{\text {cris }}(V)} \Gamma_{h}(V) \sum_{i=0}^{p-2} a_{i} \Lambda_{i},
$$

où $a_{i} \in \mathbb{Q}_{p}$ et $\Lambda_{i}=\mathbb{Z}_{p}\left[\left[\Gamma_{1}\right]\right] \delta_{i}$.

La conjecture $C_{\mathrm{Iw}}\left(K_{\infty} / K, V\right)$ est vraie si et seulement si $a_{i} \in \mathbb{Z}_{p}^{\times}$, c'est-à-dire si et seulement si la projection de $\mathscr{H}(\Gamma)$ sur $\mathbb{Q}_{p}\left[G_{n}\right]$ envoie le $\Lambda$-module (4.23) sur $\Gamma_{h}^{*}(V) \mathbb{Z}_{p}\left[G_{n}\right]$. La description explicite de la flèche inférieure de (4.22) avec les propositions 2.17 et 4.12 entraînent que cela équivaut à dire que $\delta_{V, K_{n} / K}^{\prime}$ envoie $\operatorname{det}_{\mathbb{Z}_{p}\left[G_{n}\right]} M_{n} \otimes \operatorname{det}_{\mathbb{Z}_{p}\left[G_{n}\right]} \mathbb{R} \Gamma\left(K_{n}, T\right) \operatorname{sur} \beta_{V, K_{n} / K}(M, T)^{-1}$ ce qui équivaut à la conjecture $C_{\mathrm{EP}}\left(K_{n} / K, V\right)$ et en conséquence $C_{\mathrm{Iw}}\left(K_{\infty} / K, V\right)$ est bien équivalente à $C_{\mathrm{EP}}\left(K_{n} / K, V\right)$ et le théorème 4.4 est démontré. 
4.4. Résultats principaux. Dans ce paragraphe, on démontre la conjecture $C_{\text {Iw }}\left(K_{\infty} / K, V\right)$. Rappelons que si $V$ est une représentation cristalline dont les opposés des poids de Hodge-Tate sont tous positifs, et si $T$ est un réseau de $V$, alors le module de Wach $N(T)$ est un $\boldsymbol{A}_{K}^{+}$-module libre de rang $d=\operatorname{dim}(V)$ (voir la proposition 1.1). On pose $\boldsymbol{B}_{K}^{+}=\boldsymbol{A}_{K}^{+}[1 / p]$ et $\boldsymbol{N}(V)=\boldsymbol{N}(T)[1 / p]$.

Théorème 4.19. Si $V$ est une représentation cristalline dont les opposés des poids de Hodge-Tate sont $0=r_{1} \leqslant r_{2} \leqslant \cdots \leqslant r_{d}=h$, et qui n'a pas de sous-quotient isomorphe à $\mathbb{Q}_{p}(m)$, alors :

(1) l'application $\varphi^{-1}$ induit un isomorphisme :

$$
i_{V}: \frac{\boldsymbol{D}(V)^{\psi=1}}{\left(\varphi^{*} \boldsymbol{N}(V)\right)^{\psi=1}} \stackrel{\sim}{\rightarrow} \bigoplus_{k=1}^{h}\left(K_{1} t^{-k} \otimes_{K} \operatorname{Fil}^{k} \boldsymbol{D}_{\text {cris }}(V)\right) .
$$

(2) On $a$ :

$$
\operatorname{det}_{\Lambda}\left(\frac{\boldsymbol{D}(T)^{\psi=1}}{\left(\varphi^{*} \boldsymbol{N}(T)\right)^{\psi=1}}\right)=\prod_{k=1}^{h}\left(\operatorname{det}_{\Lambda}\left(\mathbb{Z}_{p}[\Delta] \otimes \mathbb{Z}_{p}(-k)\right)\right)^{\operatorname{dim}_{\mathbb{Q}_{p}} \mathrm{Fil}^{k} \boldsymbol{D}_{\text {cris }}(V)}
$$

Nous montrons ce théorème un peu plus bas. Si $f, g \in \mathbb{Z}_{p}\left[\left[\Gamma_{1}\right]\right]$, on écrit $f \sim g$ si $f$ et $g$ sont associées, c'est-à-dire s'il existe $u \in \mathbb{Z}_{p}\left[\left[\Gamma_{1}\right]\right]^{\times}$tel que $f=g u$. De même, si $a, b \in \mathbb{Z}$ on écrit $a \sim_{p} b$ si $a$ et $b$ sont associés dans $\mathbb{Z}_{p}$. Si $M$ est un $\Lambda$-module de torsion et de type fini, on note $\operatorname{car}_{\Lambda}(M)$ son polynôme caractéristique. Rappelons que $\operatorname{det}_{\Lambda}(M)=\operatorname{car}_{\Lambda}(M)^{-1} \Lambda$.

Proposition 4.20. Si V est une représentation cristalline vérifiant les conditions du théorème 4.19 ci-dessus, alors :

(1) l'application $\varphi^{-1}$ induit une injection :

$$
i_{V}: \frac{\boldsymbol{D}(V)^{\psi=1}}{\left(\varphi^{*} \boldsymbol{N}(V)\right)^{\psi=1}} \hookrightarrow \bigoplus_{k=1}^{h}\left(K_{1} t^{-k} \otimes_{K} \operatorname{Fil}^{k} \boldsymbol{D}_{\text {cris }}(V)\right) .
$$

(2) On $a$ :

$$
\operatorname{car}_{\Lambda}\left(\frac{\boldsymbol{D}(T)^{\psi=1}}{\left(\varphi^{*} \boldsymbol{N}(T)\right)^{\psi=1}}\right) \mid \prod_{k=1}^{h}\left(\operatorname{car}_{\Lambda}\left(\mathbb{Z}_{p}[\Delta] \otimes \mathbb{Z}_{p}(-k)\right)\right)^{\operatorname{dim}_{\mathbb{Q}_{p}} \mathrm{Fil}^{k} \boldsymbol{D}_{\text {cris }}(V)}
$$

Preuve. La proposition 1.2 nous dit que :

$$
\left[\boldsymbol{B}_{\text {rig }, K}^{+} \otimes_{B_{K}^{+}} \boldsymbol{N}(V): \boldsymbol{B}_{\text {rig, } K}^{+} \otimes_{K} \boldsymbol{D}_{\text {cris }}(V)\right]=\left[(t / X)^{r_{1}} ; \ldots ;(t / X)^{r_{d}}\right],
$$


et on en déduit que :

$$
\boldsymbol{N}(V) \subset\left(\frac{X}{t}\right)^{h} \boldsymbol{B}_{\mathrm{rig}, K}^{+} \otimes_{K} \boldsymbol{D}_{\text {cris }}(V),
$$

et que le plongement de $\boldsymbol{B}_{\text {rig, } K}^{+}$dans $K[[t]]$ donne un isomorphisme $K[[t]] \otimes_{\boldsymbol{B}_{K}^{+}}$ $\boldsymbol{N}(V) \simeq K[[t]] \otimes_{K} \boldsymbol{D}_{\text {cris }}(V)$. Rappelons que l'on pose $q=\varphi(X) / X$. Comme $q^{h} \boldsymbol{N}(T) \subset \varphi^{*}\left(\boldsymbol{N}(T)\right.$ ) (voir proposition 1.1), on a $X^{-h} N(T) \subset \varphi(X)^{-h} \varphi^{*}(N(T)$ ) d'où :

$$
\boldsymbol{D}(T)^{\psi=1}=\left(\frac{1}{X^{h}} N(T)\right)^{\psi=1}=\left(\frac{1}{\varphi(X)^{h}} \varphi^{*}(N(T))\right)^{\psi=1} .
$$

Comme $\varphi^{-1}(1 / X) \in \boldsymbol{B}_{\mathrm{dR}}^{+}$, l'application $\varphi^{-1}$ induit une injection :

$$
\varphi^{-1}: \boldsymbol{D}(T)^{\psi=1} \rightarrow \operatorname{Fil}^{0}\left(K_{1}((t)) \otimes_{K} \boldsymbol{D}_{\text {cris }}(V)\right) .
$$

Pour alléger les notations, on pose :

$$
D=\operatorname{Fil}^{0}\left(K_{1}((t)) \otimes_{K} \boldsymbol{D}_{\text {cris }}(V)\right)=\bigoplus_{m=-\infty}^{h}\left(K_{1} t^{-m} \otimes_{K} \operatorname{Fil}^{m} \boldsymbol{D}_{\text {cris }}(V)\right),
$$

et on définit une filtration croissante de $D$ par des sous-espaces $D_{k}$ :

$$
D_{k}=\bigoplus_{m=-\infty}^{k}\left(K_{1} t^{-m} \otimes_{K} \operatorname{Fil}^{m} \boldsymbol{D}_{\text {cris }}(V)\right) .
$$

On a $D_{h}=D$ et $D_{k} / D_{k-1} \simeq K_{1} t^{-k} \otimes_{K} \operatorname{Fil}^{k} D_{\text {cris }}(V)$. On définit aussi une filtration sur $\left(X^{-h} \boldsymbol{N}(V)\right)^{\psi=1}$ par des sous-espaces $\boldsymbol{N}_{k}(V)$ en posant :

$$
N_{k}(V)=\left(\frac{1}{\varphi(X)^{k}} \varphi^{*}(N(V))\right)^{\psi=1}
$$

et $\varphi^{-1}$ induit alors une injection $N_{k}(V) \hookrightarrow D_{k}$. Pour montrer que l'application $N_{h}(V) / N_{0}(V) \rightarrow D_{h} / D_{0}$ est injective, il suffit de montrer que les applications $i_{V, k}: \boldsymbol{N}_{k}(V) / \boldsymbol{N}_{k-1}(V) \rightarrow K_{1} t^{k} \otimes_{K} \mathrm{Fil}^{k} \boldsymbol{D}_{\text {cris }}(V)$ sont injectives pour $k=1, \ldots, h$. Pour cela, soit $n_{1}, \ldots, n_{d}$ une base de $N(V)$ sur $\boldsymbol{B}_{K}^{+}$et soit :

$$
x=\frac{1}{\varphi(X)^{k}}\left(a_{1} \varphi\left(n_{1}\right)+\cdots+a_{d} \varphi\left(n_{d}\right)\right)
$$

un élément de $\operatorname{ker} i_{V, k}$. On a alors :

$$
\frac{\varphi^{-1}\left(a_{1}\right) n_{1}+\cdots+\varphi^{-1}\left(a_{d}\right) n_{d}}{X^{k}} \in t^{-k+1} K_{1}[[t]] \otimes_{K} \boldsymbol{D}_{\text {cris }}(V),
$$

et comme $n_{1}, \ldots, n_{d}$ forment une base de $K[[t]] \otimes_{K} \boldsymbol{D}_{\text {cris }}(V)$, on a $a_{i} \equiv 0 \bmod q$ dans $\boldsymbol{B}_{K}^{+}$. Si l'on écrit $a_{i}=q b_{i}$ avec $b_{i}=\sum_{j=0}^{\infty} b_{i j} X^{j}$, alors la condition $\psi(x)=x$ s'écrit :

$$
\left(\frac{b_{1}}{X}\right) \varphi\left(n_{1}\right)+\cdots+\left(\frac{b_{d}}{X}\right) \varphi\left(n_{d}\right)=q^{k-1}\left(\psi\left(\frac{b_{1}}{X}\right) n_{1}+\cdots+\psi\left(\frac{b_{d}}{X}\right) n_{d}\right) .
$$


On en déduit que $y=\sum_{i=1}^{d} \varphi^{-1}\left(b_{i 0}\right) n_{i}$ appartient à $\boldsymbol{D}_{\text {cris }}(V)^{\varphi=p^{k-1}}$. Mais il appartient aussi à Fil ${ }^{k-1} \boldsymbol{D}_{\text {cris }}(V)$ par construction de $i_{V, k}$, donc à $V(k-1)^{G_{K}}(1-k)=0$. Ceci montre que $i_{V, k}$ est injective. Comme $K_{1} t^{-k}$ est isomorphe à $K[\Delta] \otimes \mathbb{Z}_{p}(-k)$ en tant que $\Gamma$-modules, l'injectivité de $i_{V}$ implique :

$$
\operatorname{car}_{\Lambda_{\mathbb{Q}_{p}}}\left(\frac{\boldsymbol{D}(V)^{\psi=1}}{\left(\varphi^{*} \boldsymbol{N}(V)\right)^{\psi=1}}\right) \mid \prod_{k=1}^{h}\left(\operatorname{car}_{\Lambda}\left(\mathbb{Z}_{p}[\Delta] \otimes \mathbb{Z}_{p}(-k)\right)\right)^{\operatorname{dim}_{\mathbb{Q}_{p}} \mathrm{Fil}^{k} \boldsymbol{D}_{\text {cris }}(V)}
$$

D'autre part, comme $\boldsymbol{D}(T)$ est libre sur $\boldsymbol{A}_{K}$, le quotient $\boldsymbol{D}(T)^{\psi=1} /\left(\varphi^{*} \boldsymbol{N}(T)\right)^{\psi=1}$ n'a pas de $p$-torsion et son polynôme caractéristique est égal à

$$
\operatorname{car}_{\Lambda_{\mathbb{Q}_{p}}}\left(\boldsymbol{D}(V)^{\psi=1} /\left(\varphi^{*} \boldsymbol{N}(V)\right)^{\psi=1}\right) .
$$

L'assertion (2) s'en déduit.

Preuve du théorème 4.19. Nous démontrons d'abord le (2). Posons :

$$
\begin{aligned}
& \sum_{i=0}^{p-2} \delta_{i} \otimes f_{T, i}\left(\gamma_{1}-1\right)=\operatorname{car}_{\Lambda}\left(\frac{\boldsymbol{D}(T)^{\psi=1}}{\left(\varphi^{*} \boldsymbol{N}(T)\right)^{\psi=1}}\right), \\
& \sum_{i=0}^{p-2} \delta_{i} \otimes g_{T, i}\left(\gamma_{1}-1\right)=\prod_{m=1}^{h} \operatorname{car}_{\Lambda}\left(\mathbb{Z}_{p}\left[\Delta_{K}\right] \otimes \mathbb{Z}_{p}(-m)\right)^{\operatorname{dim}_{\mathbb{Q}_{p}} \operatorname{Fil}^{m} \boldsymbol{D}_{\text {cris }}(V)},
\end{aligned}
$$

où $\delta_{i}=\sum_{g \in \Delta_{K}} \chi^{-i}(g) g$. Posons aussi $f_{T}\left(\gamma_{1}-1\right)=\prod_{i=0}^{p-2} f_{T, i}\left(\gamma_{1}-1\right)$ et $g_{T}\left(\gamma_{1}-1\right)=$ $\prod_{i=0}^{p-2} g_{T, i}\left(\gamma_{1}-1\right)$. Comme :

$$
\delta_{i}\left(\mathbb{Z}_{p}(-m)\right)= \begin{cases}\mathbb{Z}_{p}(-m) & \text { si } i \equiv-m \quad \bmod p-1, \\ 0 & \text { sinon, }\end{cases}
$$

et comme $\mathbb{Z}_{p}[\Delta] \simeq \bigoplus_{i=0}^{p-2} \mathbb{Z}_{p} \delta_{i}$, on voit que pour $i=0, \ldots, p-2$, le $\mathbb{Z}_{p}\left[\left[\Gamma_{1}\right]\right]-$ module $\delta_{i}\left(\mathbb{Z}_{p}[\Delta] \otimes \mathbb{Z}_{p}(-m)\right)$ est isomorphe à $\mathbb{Z}_{p}(-m)$. Pour $i=0, \ldots, p-2$, on a donc :

$$
g_{T, i}\left(\gamma_{1}-1\right)=\prod_{m=1}^{h}\left(\gamma_{1}-\chi\left(\gamma_{1}\right)^{-m}\right)^{\operatorname{dim}_{\mathbb{Q}_{p}} \operatorname{Fil}^{m} \boldsymbol{D}_{\text {cris }}(V)},
$$

d'où l'on déduit que :

$$
g_{T, i}\left(\chi\left(\gamma_{1}\right)^{-k}-1\right) \sim_{p} p^{\left[K: \mathbb{Q}_{p}\right] t_{H}(V)} \prod_{m=1}^{h}(k-m)^{\operatorname{dim}_{\mathbb{Q}_{p}} \operatorname{Fil}^{m} \boldsymbol{D}_{\text {cris }}(V)} .
$$

Comme $t_{H}(V)+t_{H}\left(V^{*}(-h)\right)=h \operatorname{dim}_{\mathbb{Q}_{p}} V$ et comme :

$$
\operatorname{dim}_{\mathbb{Q}_{p}} \operatorname{Fil}^{m} \boldsymbol{D}_{\text {cris }}(V)+\operatorname{dim}_{\mathbb{Q}_{p}} \operatorname{Fil}^{1-m} \boldsymbol{D}_{\text {cris }}\left(V^{*}\right)=\left[K: \mathbb{Q}_{p}\right] \operatorname{dim}_{\mathbb{Q}_{p}} V,
$$


on voit que :

$$
g_{T}\left(\chi\left(\gamma_{1}\right)^{-k}-1\right) g_{T^{*}(-h)}\left(\chi\left(\gamma_{1}\right)^{k-h-1}-1\right) \sim_{p}\left(p^{h} \frac{\Gamma^{*}(k)}{\Gamma^{*}(k-h)}\right)^{\left[K_{1}: \mathbb{Q}_{p}\right] \operatorname{dim}(V)} .
$$

D'autre part, comme :

$$
\left(\frac{\boldsymbol{D}(T(k))^{\psi=1}}{\left(\varphi^{*} \boldsymbol{N}(T)(k)\right)^{\psi=1}}\right)^{\Gamma_{1}}=0
$$

si $k \notin[1, h]$, on a :

$$
f_{T}\left(\chi\left(\gamma_{1}\right)^{-k}-1\right)=\left[\boldsymbol{D}(T(k))_{\Gamma_{1}}^{\psi=1}:\left(\varphi^{*} \boldsymbol{N}(T)(k)\right)_{\Gamma_{1}}^{\psi=1}\right]
$$

et le corollaire 3.10 donne :

$$
f_{T}\left(\chi\left(\gamma_{1}\right)^{-k}-1\right) f_{T^{*}(-h)}\left(\chi\left(\gamma_{1}\right)^{k-h-1}-1\right) \sim_{p}\left(p^{h} \frac{\Gamma^{*}(k)}{\Gamma^{*}(k-h)}\right)^{\left[K_{1}: \mathbb{Q}_{p}\right] \operatorname{dim}(V)} .
$$

Comme la divisibilité $f_{T, i}\left(\gamma_{1}-1\right) \mid g_{T, i}\left(\gamma_{1}-1\right)$ est démontrée dans la proposition 4.20, les formules (4.24) et (4.25) entraînent :

$$
f_{T}\left(\chi\left(\gamma_{1}\right)^{-k}-1\right) \sim_{p} g_{T}\left(\chi\left(\gamma_{1}\right)^{-k}-1\right)
$$

pour tout $k \notin[1, h]$ et donc $f_{T}\left(\gamma_{1}-1\right) \sim g_{T}\left(\gamma_{1}-1\right)$ et l'assertion (2) est démontrée.

Montrons maintenant le (1). Si on note $Y$ le conoyau de l'injection :

$$
\frac{\boldsymbol{D}(V)^{\psi=1}}{\left(\varphi^{*} \boldsymbol{N}(V)\right)^{\psi=1}} \hookrightarrow \bigoplus_{k=1}^{h}\left(K_{1} t^{-k} \otimes_{K} \operatorname{Fil}^{k} \boldsymbol{D}_{\text {cris }}(V)\right),
$$

alors $\operatorname{det}_{\Lambda_{\mathbb{Q}_{p}}}(Y)=\Lambda_{\mathbb{Q}_{p}}$ par le (2), d'où $Y=0$ car $\Lambda_{\mathbb{Q}_{p}}$ est un anneau principal, ce qui montre le (1).

Corollaire 4.21. Si $k \notin[1, h]$, alors le conoyau de l'application :

$$
\Omega_{T, k, 1}^{\varepsilon}: \mathscr{D}(T(k))_{\Gamma_{1}} \rightarrow H_{\mathrm{Iw}}^{1}\left(K_{1}, T(k)\right)_{\Gamma_{1}}
$$

est isomorphe à :

$$
\bigoplus_{m=1}^{h}\left((\mathbb{Z} /(k-m) p \mathbb{Z})\left[\Delta_{K}\right]\right)^{\operatorname{dim}_{\mathbb{Q} p} \operatorname{Fil}^{m} \boldsymbol{D}_{\text {cris }}(V)} .
$$

En particulier,

$$
\operatorname{det}_{\mathbb{Z}_{p}\left[\Delta_{K}\right]}\left(\Omega_{T, k, 1}^{\varepsilon}\right)=p^{\left[K: \mathbb{Q}_{p}\right] t_{H}(V)} \prod_{m=1}^{h}(k-m)^{\operatorname{dim}_{\mathbb{Q}_{p}} \operatorname{Fil}^{m} \boldsymbol{D}_{\text {cris }}(V)} \mathbb{Z}_{p}\left[\Delta_{K}\right] .
$$


Nous pouvons maintenant démontrer le résultat principal de cet article.

Théorème 4.22. Si $V$ est une représentation cristalline de $G_{K}$, alors :

(1) la conjecture $C_{\mathrm{IW}}\left(K_{\infty} / K, V\right)$ est vraie;

(2) la conjecture $C_{\mathrm{EP}}(L / K, V)$ est vraie pour toute extension $L / K$ contenue dans $K_{\infty}$

Preuve. Comme la conjecture $C_{\mathrm{Iw}}\left(K_{\infty} / K, V\right)$ est stable par suites exactes et comme le cas $V=\mathbb{Q}_{p}(m)$ a été démontré dans [Per94, page 143], on peut supposer que $V$ n'a pas de sous-quotient fixé par $H_{K}$. De plus, $C_{\mathrm{Iw}}\left(K_{\infty} / K, V\right)$ est manifestement stable par twist. Pour fixer les idées, on va donc supposer que $V$ satisfait aux hypothèses du théorème 4.19 et on va montrer $C_{\mathrm{Iw}}\left(K_{\infty} / K, V\right)$ pour $k \notin[1, h]$. Sous ces hypothèses, $H_{\mathrm{IW}}^{1}(K, T(k))^{\Gamma_{1}}=0$, le groupe $H_{\mathrm{Iw}}^{2}(K, T(k))$ est fini et pour $n=1$ le diagramme (4.19) du paragraphe 4.3 s'écrit :

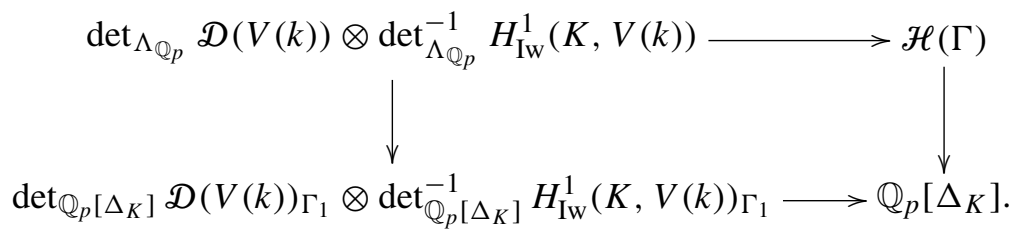

La première ligne de ce diagramme est induite par l'application

$$
\operatorname{Exp}_{V(k), k}^{\varepsilon}=(-1)^{k} \mathrm{Tw}_{V, k}^{\varepsilon} \circ \operatorname{Exp}_{V, 0}^{\varepsilon} \circ\left(\partial^{k} \otimes e_{k}\right)
$$

et le théorème 4.2 (la conjecture $\delta_{\mathbb{Q}_{p}}(V)$ de Perrin-Riou) entraîne que l'image de $\operatorname{det}_{\Lambda} \mathscr{D}(T(k)) \otimes \operatorname{det}_{\Lambda} \mathbb{R} \Gamma(K, T(k))$ dans $\mathscr{H}(\Gamma)$ s'écrit sous la forme

$$
\prod_{j=1}^{h}\left(\ell_{k-j}\right)^{\operatorname{dim}_{\mathbb{Q}_{p}} \operatorname{Fil}^{j} D_{\text {cris }}(V)} \sum_{i=0}^{p-2} a_{i} \Lambda_{i},
$$

où $a_{i} \in \mathbb{Q}_{p}$ et $\Lambda_{i}=\mathbb{Z}_{p}\left[\left[\Gamma_{1}\right]\right] \delta_{i}$. Pour conclure, il suffit de montrer que $a_{i} \in \mathbb{Z}_{p}^{*}$ pour $i=0, \ldots, p-2$. Le (2) de la proposition 3.3 montre que la deuxième ligne de (4.26) est induite par $(-1)^{k} \Omega_{T, k}^{\varepsilon}\left((\sigma \otimes \varphi)^{-1} \circ\left(\partial^{k} \otimes e_{k}\right)\right)$ et on en déduit que l'image de $\operatorname{det}_{\mathbb{Z}_{p}\left[\Delta_{K}\right]} \mathscr{D}(T(k))_{\Gamma_{1}} \otimes \operatorname{det}_{\mathbb{Z}_{p}\left[\Delta_{K}\right]}^{-1} H_{\mathrm{Iw}}^{1}(K, T(k))_{\Gamma_{1}}$ dans $\mathbb{Q}_{p}\left[\Delta_{K}\right]$ est égale à $\prod_{j=1}^{h}(k-j)^{\operatorname{dim}_{\mathbb{Q}_{p}} \operatorname{Fil}^{j} D_{\text {cris }}(V)} \sum_{i=0}^{p-2} a_{i} \delta_{i} \mathbb{Z}_{p}$.

D'autre part, comme le $\varphi$-module filtré $\boldsymbol{D}_{\text {cris }}(V)$ est admissible, on a $[M$ : $\varphi(M)]=p^{\left[K: \mathbb{Q}_{p}\right] t_{H}(V)}$ et en comparant avec la formule du corollaire 4.21, on obtient que $a_{i} \in \mathbb{Z}_{p}^{*}$ et la conjecture $C_{\mathrm{Iw}}\left(K_{\infty} / K, V\right)$ est démontrée.

Le théorème 4.3 et la proposition 2.21 montrent finalement que la conjecture $C_{\mathrm{EP}}(L / K, V)$ est vraie pour toute extension $L / K$ contenue dans $K_{\infty}$. 
Corollaire 4.23. Si $V$ est une représentation cristalline de $G_{K}$, alors :

(1) la conjecture $C_{\mathrm{EP}}(L, V)$ est vraie pour toute extension $L / K$ contenue dans $\mathbb{Q}_{p}^{\mathrm{ab}}$.

(2) la conjecture $C_{\mathrm{EP}}(K, V(\eta))$ est vraie pour tout caractère de Dirichlet $\eta$ de $\Gamma$.

Preuve. C'est une conséquence immédiate de la proposition 2.21.

\section{Références}

[Ben00] D. Benois, Iwasawa theory of crystalline representations. Duke Math. J. 104 (2) (2000), 211-267. Zbl 0996.11072 MR 1773559

[BN02] D. Benois, T. Nguyen Quang Do, Les nombres de Tamagawa locaux et la conjecture de Bloch et Kato pour les motifs $\mathbb{Q}(m)$ sur un corps abélien. Ann. Sci. École Norm. Sup. (4) 35 (5) (2002), 641-672. Zbl 1125.11351 MR 1951439

[Ber03] L. Berger, Bloch and Kato's exponential map : three explicit formulas. Doc. Math., Extra Vol. (2003), 99-129. Zbl 1064.11077 MR 2046596

[Ber04] L. Berger, Limites de représentations cristallines. Compositio Math. 140 (6) (2004), 1473-1498. Zbl 1071.11067 MR 2098398

[BK90] S. Bloch, K. Kato, L-functions and Tamagawa numbers of motives. In The Grothendieck Festschrift, Vol. I, Progr. Math. 86, Birkhäuser, Boston, MA, 1990, 333-400. Zbl 0768.14001 MR 1086888

[BF96] D. Burns, M. Flach, Motivic L-functions and Galois module structure. Math. Ann. 305 (1) (1996), 65-102. Zbl 0867.11081 MR 1386106

[BF01] D. Burns, M. Flach, Tamagawa numbers for motives with (non-commutative) coefficients. Doc. Math. 6 (2001), 501-570. Zbl 1052.11077 MR 1884523

[BG03] D. Burns, C. Greither, On the equivariant Tamagawa number conjecture for Tate motives. Invent. Math. 153 (2) (2003), 303-359. Zbl 02001021 MR 1992015

[BF04] D. Burns, M. Flach, On the equivariant Tamagawa number conjecture for Tate motives II. Doc. Math., Extra Vol. (2006), 133-163. Zbl 05165896 MR 2290586

[CC99] F. Cherbonnier, P. Colmez, Théorie d'Iwasawa des représentations $p$-adiques d'un corps local. J. Amer. Math. Soc. 12 (1) (1999), 241-268. Zbl 0933.11056 MR 1626273

[Co198] P. Colmez, Théorie d'Iwasawa des représentations de de Rham d'un corps local. Ann. of Math. (2) 148 (2) (1998), 485-571. Zbl 0928.11045 MR 1668555

[Co199a] P. Colmez, Représentations cristallines et représentations de hauteur finie. J. Reine Angew. Math. 514 (1999), 119-143. Zbl 01343282 MR 1711279

[Co199b] P. Colmez, Fonctions L p-adiques. Astérisque 266 (2000), Exp. No. 851, 3, 21-58. Zbl 0964.11055 MR 1772669

[Del73] P. Deligne, Les constantes des équations fonctionnelles des fonctions $L$. In Modular functions of one variable, II (Proc. Internat. Summer School, Univ. Antwerp, Antwerp, 1972), Lecture Notes in Math. 349, Springer-Verlag, Berlin 1973, 501-597. Zbl 0271.14011 MR 0349635 
[Del87] P. Deligne, Le déterminant de la cohomologie. In Current trends in arithmetical algebraic geometry (Arcata, Calif., 1985), Contemp. Math., 67, Amer. Math. Soc., Providence, RI, 1987, 93-177. Zbl 0629.14008 MR 0902592

[Fon91] J.-M. Fontaine, Représentations $p$-adiques des corps locaux. In The Grothendieck Festschrift, Vol. II, Progr. Math., 87, Birkhäuser, Boston, MA, 1990, 249-309. Zbl 0743.11066 MR 1106901

[Fon94a] J.-M. Fontaine, Le corps des périodes p-adiques. Astérisque 223 (1994), 59-111. Zbl 0940.14012 MR 1293971

[Fon94b] J.-M. Fontaine, Représentations p-adiques semi-stables. Astérisque 223 (1994), 113-184. Zbl 0865.14009 MR 1293972

[FP94] J.-M. Fontaine, B. Perrin-Riou, Autour des conjectures de Bloch et Kato ; cohomologie galoisienne et valeurs de fonctions L. In Motives (Seattle, WA, 1991), Proc. Sympos. Pure Math. 55, Part 1, Amer. Math. Soc., Providence, RI, 1994, 599-706. Zbl 0821.14013 MR 1265546

[Her98] L. Herr, Sur la cohomologie galoisienne des corps $p$-adiques. Bull. Soc. Math. France 126 (4) (1998), 563-600. Zbl 0967.11050 MR 1693457

[Her01] L. Herr, Une approche nouvelle de la dualité locale de Tate. Math. Ann. 320 (2) (2001), 307-337. Zbl 01663001 MR 1839766

[Kat93a] K. Kato, Lectures on the approach to Iwasawa theory for Hasse-Weil $L$-functions via $B_{d R}$. I. In Arithmetic algebraic geometry (Trento, 1991), Lecture Notes in Math. 1553, Springer-Verlag, Berlin 1993, 50-163. Zbl 0815.11051 MR 1338860

[Kat93b] K. Kato, Lectures on the approach to Iwasawa theory for Hasse-Weil $L$-functions via $B_{d R}$, part II. Preprint 1993.

[Kat93c] K. Kato, Iwasawa theory and p-adic Hodge theory. Kodai Math. J. 16 (1) (1993), 1-31. Zbl 0798.11050 MR 1207986

[KKT96] K. Kato, M. Kurihara, T. Tsuji, Local Iwasawa theory of Perrin-Riou and syntomic complexes. Preprint 1996.

[KM76] F. Knudsen, D. Mumford, The projectivity of the moduli space of stable curves I : Preliminaries on "det” and "Div". Math. Scand. 39 (1) (1976), 19-55. Zbl 0343.14008 MR 0437541

[Laz62] M. Lazard, Les zéros des fonctions analytiques d'une variable sur un corps valué complet. Inst. Hautes Études Sci. Publ. Math. 14 (1962), 47-75. Zbl 0119.03701 MR 0152519

[Mat92] H. Matsumura, Commutative ring theory. Cambridge Stud. Adv. Math. 8, Cambridge University Press, Cambridge 1989. Zbl 0666.13002 MR 1011461

[Nek02] J. Nekovář, Selmer complexes. Astérisque 310 (2006). Zbl 05161833 MR 2333680

[Per92] B. Perrin-Riou, Théorie d'Iwasawa et hauteurs p-adiques. Invent. Math. 109 (1992), 137-185. Zbl 0781.14013 MR 1168369

[Per94] B. Perrin-Riou, Théorie d'Iwasawa des représentations $p$-adiques sur un corps local. Invent. Math. 115 (1) (1994), 81-161. Zbl 0838.11071 MR 1248080

[Per95] B. Perrin-Riou, Fonctions $L$-adiques des représentations $p$-adiques. Astérisque 229 (1995). Zbl 0845.11040 MR 1327803 
[Ser68] J.-P. Serre, Corps locaux. Deuxième édition. Publications de l'Université de Nancago, No. VIII, Hermann, Paris 1968. Zbl 1095.11504 MR 0354618

[Tat67] J. Tate, Fourier analysis in number fields, and Hecke's zeta-function. In Algebraic Number Theory (Proc. Instructional Conf., Brighton, 1965), Thompson, Washington, D.C., Academic Press, London 1967, 305-347. Zbl 0153.07403 MR 0217026

Received September 28, 2006

Denis Benois, Faculté des Sciences et Techniques, Université de Besançon, 16 route de Gray, 25030 Besançon Cedex, France

E-mail: denis.benois@math.univ-fcomte.fr

Laurent Berger, Université de Lyon, UMPA ENS Lyon, 46 allée d'Italie, 69364 Lyon

Cedex 07, France

E-mail: laurent.berger@umpa.ens-lyon.fr; www.umpa.ens-lyon.fr/ lberger/ 